\title{
Teoria de forma normal para campos vetoriais reversíveis equivariantes
}

Iris de Oliveira Zeli 

SERVIÇO DE PÓS-GRADUAÇÃO DO ICMC-USP

Data de Depósito:

Assinatura:

\title{
Teoria de forma normal para campos vetoriais reversíveis equivariantes
}

\author{
Iris de Oliveira Zeli
}

Orientadora: Profa. Dra. Miriam Garcia Manoel

Tese apresentada ao Instituto de Ciências Matemáticas e de Computação - ICMC-USP, como parte dos requisitos para obtenção do título de Doutor em Ciências - Matemática. VERSÃO REVISADA

USP - São Carlos

Junho de 2013 
Ficha catalográfica elaborada pela Biblioteca Prof. Achille Bassi e Seção Técnica de Informática, ICMC/USP, com os dados fornecidos pelo(a) autor(a)

\begin{tabular}{|c|c|}
\hline \multirow[t]{3}{*}{$d 49 t$} & $\begin{array}{l}\text { de Oliveira Zeli, Iris } \\
\text { Teoria de forma normal para campos vetoriais } \\
\text { reversiveis equivariantes / Iris de Oliveira Zeli; } \\
\text { orientadora Miriam Garcia Manoel. -- São Carlos, } \\
2013 . \\
\quad 90 \mathrm{p} .\end{array}$ \\
\hline & $\begin{array}{l}\text { Tese (Doutorado - Programa de Pós-Graduação em } \\
\text { Matemática)-- Instituto de Ciências Matemáticas e } \\
\text { de Computação, Universidade de São Paulo, } 2013 .\end{array}$ \\
\hline & $\begin{array}{l}\text { 1. forma normal. 2. Belitskii. 3. reversível } \\
\text { equivariante . 4. transversal completa. 5. campo } \\
\text { vetorial. I. Garcia Manoel, Miriam, orient. II. } \\
\text { Título. }\end{array}$ \\
\hline
\end{tabular}


Tenho a impressão de ter sido uma criança brincando à beira mar, divertindo-me em descobrir uma pedrinha mais lisa ou uma concha mais bonita que as outras, enquanto o imenso oceano da verdade continua misterioso diante dos meus olhos. (Isaac Newton) 



\section{Agradecimentos}

Em primeiro lugar agradeço a Cristo, criador e consumador de todas as coisas, por tudo o que me concedeu até aqui. Por uma família linda e por oportunidades como esta, de concluir o doutorado.

Ao meu esposo Rodrigo, pelo amor, amizade, cumplicidade, carinho, companheirismo, paciência e por acreditar em mim até mais do que eu mesma! Obrigada por ter estado ao meu lado em todo esse tempo, me entender e por renunciar horas comigo para que eu me dedicasse à matemática. Eu não teria chegado até aqui sem você. Obrigada por andar comigo sempre! Depois de 7 anos juntos não tenho dúvidas de que ninguém mais além de você, seria a pessoa ideal para estar ao meu lado. Te amo muito!

Agradeço aos meus pais, José Mauro e Lucila, pela educação que me deram, pelo sustento, pela força e encorajamento em todos os momentos, pelas orações e, principalmente, pelo amor incondicional demonstrado em cada atitude. Com eles eu aprendi que mesmo tendo pouco, e nem sempre do jeito que gostaríamos, é possível crescer e alcançar os nossos objetivos de forma digna. Ao meu irmão Jéfter e minhas irmãs Iandra e Iara. Aos meus lindíssimos e meigos sobrinhos que tanto amo: Caroline, Gabriela, Samuel e Ester. Aos membros "agregados"da família Petrônio, Krys, Leonardo, Liamara e Dona Verônica. Apesar da distância e da imensa saudade, vocês foram e sempre serão o meu maior tesouro.

À amiga única e insubstituível Gerusa Said, pela companhia (online) em muitas madrugadas que resultaram em muitas gargalhadas, e por todos os excelentes conselhos. 
À todos os professores e funcionários do ICMC, pela disposição em ajudar. Em especial, à minha orientadora Miriam Manoel, pelo tema proposto, dedicação e pela seriedade durante toda a orientação. Foram 6 anos, entre metrado e doutorado, de muito aprendizado. Agradeço pelo referencial de excelente profissional que sempre foi para mim.

À professora e amiga Patrícia Baptistelli, pela participação neste trabalho, e longas conversas durante o tempo em que esteve em São Carlos para cursar o pós doutorado.

Ao professor Maurício Lima, pelas ideias sugeridas e contribuição para a escolha do tema estudado.

Ao professor Marcelo Escudeiro, pela colaboração e sugestão de um assunto que resultou no Capítulo 4 desta tese.

Ao professor Alan Araújo, um dos principais responsáveis pela minha vinda para São Carlos, pela amizade, apoio e bons conselhos sobre a matemática.

À professora Sandra Godoy, pelo carinho que sempre teve por mim, por sempre me incentivar e pela oportunidade de estágios PAE. Certamente, os estágios foram totalmente relevantes para a minha formação profissional.

Aos amigos de mestrado e doutorado que encontrei neste instituto e que vou levar comigo. Pelas horas tristes e alegres, pela companhia em várias madrugadas, pela ajuda que me deram quando achei que não conseguiria avançar, pelas piadas, risadas, cafezinhos e pelos churrascos! Com vocês os momentos difíceis foram muito mais fáceis!

Agradeço a todos aqueles que de alguma forma, direta ou indiretamente, me ajudaram a permanecer de pé e confiante. Com certeza, família e verdadeiros amigos são pilares fundamentais para o sucesso na vida. Dedico a minha conquista a todos vocês!

Ao Proex pelo apoio financeiro concedido para participação em diversos eventos.

Enfim, agradeço à CAPES e ao CNPq pelo suporte financeiro concedido ao longo desses 4 anos de doutorado. 
Neste trabalho, apresentamos um método algébrico para obter formas normais de campos vetoriais reversíveis equivariantes. Adaptamos o método clássico de Belitskii-Elphick, usando ferramentas da teoria invariante para estabelecer fórmulas que consideram as simetrias e antissimetrias como ponto de partida. Mostramos que este método, mesmo sem simetrias, possui uma estreita relação com o método da transversal completa da teoria de singularidades. Com as ferramentas desenvolvidas nesta tese, a forma normal obtida é uma série formal que não depende do cálculo do kernel do chamado operador homológico. Formas normais para duas classes de campos, ressonantes e não ressonantes, são apresentadas, para diferentes representações do grupo $\mathbf{Z}_{2} \times \mathbf{Z}_{2}$ cuja linearização tem uma parte nilpotente de dimensão 2 e uma parte semi-simples com autovalores puramente imaginários. 

We give an algebraic method to obtain normal forms of reversible equivariant vector fields. We adapt the classical method by Belitskii-Elphick using tools from invariant theory to establish formulae that take symmetries into account as a starting point. We show that this method, even without symmetries, has a close relation to complete transversal of singularities theory. Applying the method developed in this thesis, the resulting normal form is a formal series which does not depend of the computation of the kernel of the so called homologic operator. Normal forms of two classes of non-resonant and resonant cases are presented, for different representations of the group $\mathbf{Z}_{2} \times \mathbf{Z}_{2}-$ with linearization having a 2 -dimensional nilpotent part and a semisimple part with purely imaginary eigenvalues. 

Notações

Introdução 1

\begin{tabular}{lll}
\hline 1 & Preliminares da teoria reversível equivariante & 7
\end{tabular}

1.1 Representação de grupos e integral de Haar . . . . . . . . . . . . . . . . . 8

1.2 Invariantes e equivariantes . . . . . . . . . . . . . . . . . . . . . . . 10

$1.3 \quad \mathrm{O}$ contexto com simetrias e antissimetrias $\ldots \ldots \ldots \ldots \ldots$

1.4 Operadores de Reynolds e algoritmos . . . . . . . . . . . . . . . . . . . . 13

1.5 Séries de Hilbert e fórmulas de Molien . . . . . . . . . . . . . . . . . . . . 16

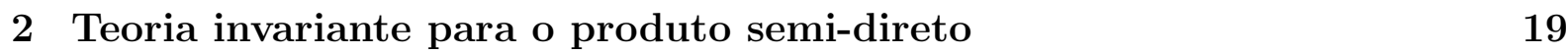

2.1 Invariantes para o produto semi-direto . . . . . . . . . . . . . . 20

2.2 Representação diagonal no contexto invariante . . . . . . . . . . . . . 22

2.3 Séries de Hilbert e fórmulas de Molien para a ação diagonal . . . . . . . . . . 24

\begin{tabular}{|lll}
3 & Forma normal reversível equivariante & 31
\end{tabular}

3.1 o método de Belitskil . . . . . . . . . . . . . . . . . . . . . . . . . . 32

3.2 Propriedades do operador homológico . . . . . . . . . . . . . . . . . . . . 34 
3.3 Forma normal reversível equivariante . . . . . . . . . . . . . . . . . . 36

4 A forma normal de Belitskii-Elphick e o método da transversal completa 43

4.1 Preliminares . . . . . . . . . . . . . . . . . . . . . . . . . . . . . . . . . . . . . . 44

$4.2 \quad \mathrm{O}$ contexto sem simetrias $\ldots \ldots \ldots \ldots \ldots$

$4.3 \quad$ O contexto reversível equivariante $\ldots \ldots \ldots \ldots$

5 Campos de vetores $\mathrm{Z}_{2} \times \mathrm{Z}_{2}$-reversíveis-equivariantes 53

5.1 Pares de involuções que anti-comutam com o campo de vetores . . . . . . . 55

5.2 Cálculo da forma normal . . . . . . . . . . . . . . . . . . . . . 57

5.3 Formas normais $\mathbf{Z}_{2} \times \mathbf{Z}_{2}-$ reversíveis-equivariantes $\ldots \ldots \ldots$. . . . . . . 59

5.3 .1 Caso não ressonante . . . . . . . . . . . . . . . . . . . . . . 59

5.3.2 Ressonância do tipo $\left(n_{1}: n_{2}\right)$ em $\mathbb{R}^{2} \times \mathbb{C}^{2} \ldots \ldots \ldots$. . . . . . . 62

5.3.3 Ressonância do tipo $\left(n_{1}: n_{2}: 0\right)$ em $\mathbb{R}^{2} \times \mathbb{C}^{3} \ldots \ldots$. . . . . . 68

5.3.4 Ressonância do tipo $\left(n_{1}: n_{2}: 0\right)$ em $\mathbb{R}^{2} \times \mathbb{C}^{n} \ldots \ldots \ldots$. . . . . . 72

5.3.5 Ressonância do tipo $\left(n_{1}: n_{2}-m_{1}: m_{2}\right)$ em $\mathbb{R}^{2} \times \mathbb{C}^{4} \ldots \ldots . . . .76$

\begin{tabular}{ll}
\hline Bibliografia & 83
\end{tabular}

\begin{tabular}{lr}
\hline Índice Remissivo & 89
\end{tabular} 


\section{Lista de Tabelas}

$5.1 \quad$ Formas normais de campos $\mathbf{Z}_{2} \times \mathbf{Z}_{2}$-reversíveis-equivariantes $\ldots . . .66$

5.2 Geradores em $\mathbb{R}^{2} \times \mathbb{C}^{2} \ldots \ldots \ldots \ldots \ldots$

$5.3 \quad$ Geradores em $\mathbb{R}^{2} \times \mathbb{C}^{3} \ldots \ldots \ldots \ldots \ldots \ldots \ldots$

$5.4 \quad$ Geradores em $\mathbb{R}^{2} \times \mathbb{C}^{n} \ldots \ldots \ldots \ldots \ldots \ldots \ldots$ 

$\Gamma$ : Grupo das simetrias e antissimetrias de um sistema;

$\Gamma_{+}$: Subgrupo de $\Gamma$ de índice 2 , formado pelas simetrias de $\Gamma$;

$\delta$ : Antissimetria fixada de $\Gamma$;

$\Gamma_{-}=\delta \Gamma_{+}:$Subconjunto formado pelas antissimetrias de $\Gamma$;

$\sigma:$ Homomorfismo de $\Gamma$ em $\mathbf{Z}_{2}$;

$(\rho, V)$ : Representação de $\Gamma$ em $V$ (ou simplesmente $\rho$ );

$\rho_{\sigma}:$ Representação $\sigma$-dual de $\rho$;

$\int_{\Gamma}:$ Integral de Haar normalizada sobre $\Gamma$;

$\mathcal{P}_{V}:$ Anel dos germes de funções polinomiais $f: V, 0 \rightarrow \mathbb{R}$;

$\mathcal{P}_{V}(\Gamma)$ : Anel dos germes de funções polinomiais $f: V, 0 \rightarrow \mathbb{R} \Gamma$-invariantes;

$\mathcal{P}_{V}^{k}:$ Espaço vetorial dos germes de polinômios homogêneos $f: V, 0 \rightarrow \mathbb{R}$ de grau $k$;

$\mathcal{P}_{V}^{k}(\Gamma)$ : Espaço vetorial dos polinômios homogêneos $f: V, 0 \rightarrow \mathbb{R} \Gamma$-invariantes de grau $k$;

$\overrightarrow{\mathcal{P}}_{V, W}:$ Módulo sobre $\mathcal{P}_{V}$ dos germes de aplicações polinomiais $g: V, 0 \rightarrow W$; 
$\overrightarrow{\mathcal{P}}_{V, W}(\Gamma)$ : Módulo sobre $\mathcal{P}_{V}(\Gamma)$ dos germes de aplicações polinomiais $g: V, 0 \rightarrow W$ $\Gamma$-equivariantes;

$\overrightarrow{\mathcal{P}}_{V, W}^{k}:$ Espaço vetorial dos germes de aplicações polinomiais homogêneas $g: V, 0 \rightarrow W$ de grau $k$;

$\overrightarrow{\mathcal{P}}_{V, W}^{k}(\Gamma):$ Espaço vetorial dos germes de aplicações polinomiais homogêneas $g: V, 0 \rightarrow W$ $\Gamma$-equivariantes de grau $k$;

$\mathcal{Q}_{V}(\Gamma)$ : Módulo sobre $\mathcal{P}_{V}(\Gamma)$ dos germes de funções polinomiais $f: V, 0 \rightarrow \mathbb{R} \Gamma$-antiinvariantes;

$\mathcal{Q}_{V}^{k}(\Gamma)$ : Espaço vetorial dos germes de polinômios homogêneas $f: V, 0 \rightarrow \mathbb{R} \Gamma$-antiinvariantes de grau $k$;

$\overrightarrow{\mathcal{Q}}_{V}(\Gamma)$ : Módulo sobre $\mathcal{P}_{V}(\Gamma)$ dos germes de aplicações polinomiais $g: V, 0 \rightarrow V \Gamma$-reversíveisequivariantes;

$\overrightarrow{\mathcal{Q}}_{V}^{k}(\Gamma)$ : Espaço vetorial dos germes de aplicações polinomiais homogêneas $g: V, 0 \rightarrow V$ $\Gamma$-reversíveis-equivariantes de grau $k$;

$\mathcal{E}_{V}:$ Anel dos germes de funções $f: V, 0 \rightarrow \mathbb{R}$

$\mathcal{E}_{V}(\Gamma):$ Anel dos germes de funções $f: V, 0 \rightarrow \mathbb{R} \Gamma$-invariantes;

$\overrightarrow{\mathcal{E}}_{V, W}:$ Módulo sobre $\mathcal{E}_{V}(\Gamma)$ dos germes de aplicações $g: V, 0 \rightarrow W$;

$\overrightarrow{\mathcal{E}}_{V, W}(\Gamma)$ : Módulo sobre $\mathcal{E}_{V}(\Gamma)$ dos germes de aplicações $g: V, 0 \rightarrow W \Gamma$-equivariantes;

$\mathcal{F}_{V}(\Gamma)$ : Módulo sobre $\mathcal{E}_{V}(\Gamma)$ dos germes de funções $f: V, 0 \rightarrow \mathbb{R} \Gamma$-anti-invariantes;

$\overrightarrow{\mathcal{F}}_{V}(\Gamma)$ : Módulo sobre $\mathcal{E}_{V}(\Gamma)$ dos germes de aplicações $g: V, 0 \rightarrow V \Gamma$-reversíveisequivariantes;

$\chi_{V}$ : Caracter da representação de $\Gamma$ em $V$;

$\Phi_{V}^{\Gamma}$ : Série de Hilbert para o anel $\mathcal{P}_{V}(\Gamma) ;$

$\Psi_{V, W}^{\Gamma}$ : Série de Hilbert para o módulo $\overrightarrow{\mathcal{P}}_{V, W}(\Gamma)$

$\tilde{\Phi}_{V}^{\Gamma}$ : Série de Hilbert para o módulo $\mathcal{Q}_{V}(\Gamma) ;$ 
$\tilde{\Psi}_{V}^{\Gamma}:$ Série de Hilbert para o módulo $\overrightarrow{\mathcal{Q}}_{V}(\Gamma)$;

$A d_{L}$ : Operador homológico definido em $\mathcal{P}_{V}$

$A d_{L}^{k}:$ Restrição de $A d_{L}$ a $\overrightarrow{\mathcal{P}}_{V}^{k}$

$j^{k} g: k$-jato de $g$ em $0 \in V, 0$;

$J^{k}:$ Espaço dos $k$-jatos dos germes de aplicações polinomiais $g: V, 0 \rightarrow V, g(0)=0$;

$J^{k}\left(\Gamma_{\sigma}\right)$ : Subespaço de $J^{k}$ dos $k$-jatos $\Gamma$-reversíveis-equivariantes;

$\mathcal{M}$ : Ideal maximal de $\mathcal{E}_{V}$

$\mathcal{M}^{k}: k$-ésima potência de $\mathcal{M}$;

$\overrightarrow{\mathcal{F}}_{V k+1}(\Gamma):$ Espaço $\mathcal{M}^{k+1} \overrightarrow{\mathcal{E}}_{V} \cap \overrightarrow{\mathcal{F}}_{V}(\Gamma)$

$T \mathcal{G} \cdot h$ : Espaço tangente à órbita de $h$ segundo a ação do grupo $\mathcal{G}$;

$\operatorname{Fix}(\varphi)$ : Subespaço de pontos fixos pela involução $\varphi$. 
A teoria de forma normal tem sido desenvolvida como uma ferramenta para o estudo local de campos vetoriais $C^{\infty}$ na vizinhança de uma singularidade deste campo. A importância dessa ferramenta no estudo de bifurcação e em muitas outras direções tem motivado o desenvolvimento do objeto por vários autores há anos, desde Poincaré [53], Birkhoff [7], Dulac [28], Belitskii [6], Elphick et al. [29] e Takens [57]. O método clássico consiste de mudanças de coordenadas em torno de um ponto singular que são perturbações da identidade. O objetivo é anular a maior quantidade de termos de grau $k, k \geq 2$, da série de Taylor do campo vetorial original, obtendo um campo vetorial equivalente escrito de uma forma mais simples e mais conveniente.

Para cada $k \geq 2$, o método proposto por Belitskii [6] considera equivalência de campos vetoriais (equivalência dada pela conjugação de campos vetoriais) através de mudanças de coordenadas do tipo $I+\xi_{k}$, onde $\xi_{k}$ é um polinômio homogêneo de grau $k$. A obtenção da forma normal por este método trata do cálculo do kernel do chamado operador homológico. Este operador é definido no espaço vetorial dos polinômios homogêneos de grau $k$, a partir da linearização $L$ do campo vetorial original no ponto singular. Os elementos da base deste kernel são os termos presentes na forma normal.

A obtenção do kernel, entretanto, consiste de encontrar soluções polinomiais de cada grau $k$ de uma EDP. Elphick et al. [29] fornece uma alternativa para se obter a forma normal trocando o problema de resolver uma EDP pelo cálculo das aplicações equivariantes pela ação de um grupo a um parâmetro $\mathbf{S}$ definido como o fecho de um conjunto definido 
a partir de $L$, a saber

$$
\mathbf{S}=\overline{\left\{e^{s L^{t}}, s \in \mathbb{R}\right\}}
$$

onde $L^{t}$ denota a transposta de $L$. O reconhecimento desta $\mathbf{S}$-equivariância foi o que nos despertou interesse pelo tratamento algébrico na teoria de forma normal de Belitskii.

Paralelamente, existe o interesse na obtenção da forma normal de campos vetoriais em presença simultânea de simetrias e antissimetrias. Neste caso, o tratamento algébrico mostra vantagem, mesmo no contexto sem simetrias, pois existe uma série de ferramentas algébricas para lidarmos com estes campos vetoriais. O conjunto formado pelas simetrias e antissimetrias do campo vetorial tem estrutura de grupo, aqui denotado por $\Gamma$, e neste caso o campo de vetores é chamado $\Gamma$-reversível-equivariante. Nosso estudo começa com um homomorfismo de grupos

$$
\sigma: \Gamma \rightarrow \mathbf{Z}_{2}
$$

$\mathbf{Z}_{2}$ o grupo multiplicativo $\{1,-1\}$, que define as simetrias: se $\gamma \in \Gamma$ é simetria, $\sigma(\gamma)=1$; se $\gamma \in \Gamma$ é antissimetria, $\sigma(\gamma)=-1$. Segue que, o conjunto das simetrias, denotado por $\Gamma_{+}$, aparece naturalmente como um subgrupo normal de índice $2 \mathrm{em} \Gamma$. Campos vetoriais reversíveis equivariantes têm uma forma bem determinada que pode ser obtida a partir do conhecimento da teoria invariante do grupo $\Gamma_{+}$(veja [2]). Quando o campo possui apenas simetrias, dizemos ser puramente equivariante e em [35] encontramos uma série de resultados e uma lista completa de referências para este caso.

A ocorrência de simetrias e antissimetrias (os elementos de $\Gamma$ ) em um campo vetorial tem um efeito não apenas na existência e repetição de pontos singulares, ou na dinâmica, etc, mas também na forma das mudanças de coordenadas. Mais precisamente, a forma normal herda as simetrias e antissimetrias se as mudanças de coordenadas são equivariantes pela ação do grupo $\Gamma$. Do ponto de vista da dinâmica, simetrias levam trajetórias em trajetórias preservando a direção com o tempo, enquanto que antissimetrias revertem a direção com o tempo.

A dinâmica de sistemas puramente reversíveis (com uma única antissimetria e sem simetrias não triviais) tem sido estudada mais intensamente há mais tempo, por exemplo [14, 18, 20, 38, 39, 43]. A importância de uma antissimetria foi notada por Birkhoff [7] em um estudo do problema restrito de três corpos, e anos depois Devaney [27] apresentou definições formais sobre campos vetoriais reversíveis equivariantes. Nos últimos anos, sistemas reversíveis equivariantes se tornou objeto de estudo de muitos autores. Notamos um 
grande crescimento do número de trabalhos científicos sobre este assunto, veja por exemplo [2, 3, 4, 12, 13, 41, 42, 45], mas foi apenas em [2] e [4] que houve um estudo sistemático sobre a relação entre sistemas puramente equivariantes e reversíveis equivariantes.

Em [2], os autores desenvolvem um método para a obtenção da forma geral de campos vetoriais reversíveis equivariantes, uma vez conhecidos os geradores para os invariantes e equivariantes pela ação de $\Gamma_{+}$. Avançando nesta direção, Baptistelli e Manoel apresentam em [4] um método para determinar uma base de Hilbert para o anel dos polinômios invariantes sob ação do grupo $\Gamma$ através de uma base de Hilbert para o anel dos polinômios invariantes por $\Gamma_{+}$. Quando $\Gamma_{+}$é finitamente gerado, o software Singular [55] pode auxiliar o processo de obtenção dos invariantes pela ação de $\Gamma_{+}$, desde que seja fornecido ao programa a forma matricial dos geradores de $\Gamma_{+}$.

Muitos autores têm usado a teoria de forma normal para estudar a ocorrência de ciclos homoclínicos e heteroclínicos e famílias de órbitas periódicas tanto em sistemas puramente reversíveis quanto em sistemas reversíveis equivariantes (veja, por exemplo, [17, 44, 45, 47, 50]). Nestes trabalhos, a obtenção da forma normal truncada até grau $k$ é feita através do cálculo do kernel do operador homológico e então as condições de simetrias são impostas como um passo a posteriori. Nesses casos, muitas vezes a forma normal é truncada em um baixo grau devido às dificuldades de determinar soluções para a EDP associada, principalmente quando o sistema possui ressonância. Neste caso, a condição de ressonância afeta diretamente a forma do grupo $\mathbf{S}$ em (0.1). Sobre sistemas com ressonância citamos ainda os trabalhos [15, 16, 22, 44]. O resultado geral desta tese foi motivado por uma pergunta colocada a nós por M. F. S. Lima sobre a obtenção da forma normal desenvolvida por Belitskii no contexto reversível. Nesta direção, desenvolvemos um método mais eficaz para a obtenção da forma normal reversível equivariante usando ferramentas algébricas sem recorrer a soluções de uma EDP.

Neste trabalho, adaptamos a teoria de forma normal desenvolvida por Belitskii [6] e Elphick [29] para o contexto reversível equivariante, usando ferramentas algébricas da teoria invariante. Neste processo o grupo $\mathbf{S}$ dado em 0.1 desempenha um papel fundamental: nós mostramos que a forma normal $\Gamma$-reversível-equivariante é obtida a partir do conhecimento de geradores para os reversíveis equivariantes pelo produto semi-direto $\mathbf{S} \rtimes \Gamma$. Depois deste reconhecimento, usamos resultados em [2] como uma ferramenta para o cálculo da forma normal. Enfatizamos que essas ferramentas podem ser usadas também 
para grupos de Lie não compactos, uma vez que o anel dos invariantes e o módulo dos equivariantes pelo grupo considerado sejam finitamente gerados. A contribuição deste resultado na teoria geral é um caminho que produz uma série formal para a forma normal de Belitskii reversível equivariante.

A teoria de forma normal de Belitskii está estritamente relacionada ao método da transversal completa, desenvolvido por Bruce et al. [11 na classificação de germes em teoria de singularidades segundo o grupo $\mathcal{A}$ (mudanças de coordenadas na fonte e na meta). Este método também usa ferramentas algébricas para a classificação sem recorrer a mudanças de coordenadas. Dentre centenas de trabalhos nesta linha, citamos por exemplo os clássicos trabalhos de Wall [59, 60], Bruce et al. [10], Gaffney e du Plessis [30], Gaffney [31, e em classificação de singularidades aplicada à teoria de bifurcação citamos Golubitsky et al. [34, 35] e Melbourne [48, 49], estes nos contextos com e sem simetrias.

A motivação para compararmos este dois métodos se deu primeiramente no caso $\Gamma$-reversível-equivariante, através de uma pergunta feita por M. E. Hernandez durante nossa visita à UEM, Maringá-PR. Durante o estudo, entretanto, notamos a validade dos resultados mesmo no contexto sem simetrias. Assim, esta caracterização usando o método da transversal completa é uma forma alternativa para a obtenção da forma normal usando ferramentas da teoria de singularidades.

Esperamos que os resultados obtidos nesta tese contribuam para a teoria de forma normal reversível equivariante, uma vez que a forma normal obtida é uma série formal que não depende do cálculo do kernel do operador homológico. Como parte de aplicações calculamos formas normais para duas classes de campos vetoriais, ressonantes e não ressonantes, em sistemas reversíveis equivariantes. Estas aplicações generalizam algumas formas normais apresentadas em [44].

A seguir, descrevemos o conteúdo de cada capítulo.

No Capítulo 1 apresentamos conceitos básicos da teoria invariante que podem ser encontrados em [35]. Introduzimos os principais resultados da teoria reversível equivariante que são usados ao longo deste trabalho, dentre eles o Algoritmo 1.4.1 e o Teorema 1.4.2 que se encontram em [2, 4].

No Capítulo 2 formalizamos a teoria invariante para o produto semi-direto de dois grupos arbitrários. O objetivo é descrever formalmente as ferramentas necessárias usadas 
nesta tese e, principalmente, nos Capítulos 3 e 5 , neste último para a construção de formas normais reversíveis equivariantes.

O Capítulo 3 é dedicado aos resultados centrais desta tese. Introduzimos os conceitos básicos da teoria de forma normal desenvolvida por Belitskii [6] e Elphick [29], provamos algumas propriedades do operador homológico no contexto reversível equivariante e então provamos os principais resultados, Teoremas 3.3.1 e 3.3.5.

No Capítulo 4 descrevemos a relação entre a teoria clássica de forma normal e o método da transversal completa. Apresentamos uma breve introdução da teoria de singularidades e depois definimos uma relação de equivalência específica. Os resultados são apresentados primeiro no contexto sem simetrias, Teorema 4.2.3, e em seguida no contexto reversível equivariante, Teorema 4.3 .3 .

Finalmente, no Capítulo 5 aplicamos os resultados do Capítulo 3 para obter a forma normal de campos vetoriais reversíveis equivariantes por um grupo do tipo $\mathbf{Z}_{2} \times \mathbf{Z}_{2}$, gerado por um par de involuções lineares que, neste caso, são reversibilidades. Apresentamos a classificação desses pares de involuções e adaptamos técnicas da teoria reversível equivariante para serem usadas no cálculo das formas normais. Para estes cálculos, consideramos campos vetoriais cuja linearização tem uma parte nilpotente de dimensão 2 e uma parte com autovalores puramente imaginários. 



\begin{tabular}{c|c|}
\hline \multirow{2}{*}{} & Capítulo \\
\hline 1 \\
\hline
\end{tabular}

\section{Preliminares da teoria reversível} equivariante

Simetrias e antissimetrias podem ocorrer de forma natural em diversos fenômenos físicos. Encontramos uma série de exemplos em sistemas dinâmicos com esta propriedade, como por exemplo, sistemas da mecânica clássica, mecânica celeste, termodinâmica, etc. O conjunto formado pelas simetrias e antissimetrias de um sistema tem estrutura de grupo, aqui denotado por $\Gamma$ e, neste caso, o sistema é chamado $\Gamma$-reversível-equivariante. Quando o sistema possui apenas simetrias o chamamos de puramente equivariante. Muitos resultados sobre estes sistemas podem ser adaptados ao contexto reversível equivariante já que este pode ser visto como um tipo de sistema equivariante, como vemos na Seção 1.3 .

As equações de um sistema $\Gamma$-reversível-equivariante têm uma forma geral específica que pode ser determinada através de ferramentas algébricas desenvolvidas em [2]. Em muitos casos, esta forma geral pode ser obtida com o auxílio do programa Singular [55].

Neste capítulo introduzimos as ferramentas essenciais para o estudo de sistemas $\Gamma$-reversíveis-equivariantes. Os resultados apresentados aqui se encontram em [2, 4, 35]. Come- 
çamos na Seção 1.1 com conceitos básicos da teoria de representação de grupos. Na Seção 1.2 introduzimos os invariantes e equivariantes. Destacamos os Teoremas de Hilbert-Weyl e de Poènaru que estabelecem que o anel dos invariantes e o módulo dos equivariantes segundo a ação de um grupo de Lie compacto $\Gamma$ são finitamente gerados. Na Seção 1.3 introduzimos definições e resultados mais recentes da literatura e na Seção 1.4 destacamos dentre estes resultados o algoritmo para a obtenção de um conjunto gerador para o módulo das aplicações $\Gamma$-reversíveis-equivariantes. Finalmente, na Seção 1.5 fazemos uma breve apresentação sobre séries de Hilbert e fórmulas de Molien para grupos de Lie compactos.

\subsection{Representação de grupos e integral de Haar}

Devido à estrutura de grupo que existe no conjunto das simetrias e antissimetrias presentes em um sistema reversível equivariante, o formalismo para o estudo de diversos aspectos de tais sistemas é feito através da teoria de representação de grupos.

Seja $\Gamma$ um grupo de Lie compacto e $V$ um espaço vetorial real de dimensão finita.

Dizemos que $\Gamma$ age linearmente em $V$ se existe uma aplicação contínua $\Gamma \times V \rightarrow V$, $(\gamma, x) \mapsto \gamma x$, tal que:

(a) Para cada $\gamma \in \Gamma$, a aplicação $\rho_{\gamma}: V \rightarrow V$ definida por $\rho_{\gamma}(x)=\gamma x$ é linear;

(b) Se $\gamma_{1}, \gamma_{2} \in \Gamma$ então $\gamma_{1}\left(\gamma_{2}(x)\right)=\left(\gamma_{1} \gamma_{2}\right) x$.

A ação de $\Gamma$ em $V$ induz uma representação de $\Gamma$ em $\mathbf{G L}(V)$, espaço das aplicações lineares invertíveis $V \rightarrow V$, definida por

$$
\begin{aligned}
\rho: \Gamma & \rightarrow \mathbf{G L}(V), \\
\gamma & \mapsto \rho(\gamma)
\end{aligned}
$$

onde $\rho(\gamma) x=\rho_{\gamma}(x)$.

Naturalmente, a cada ação corresponde uma representação e vice-versa. Denotamos por $(\rho, V)$ o espaço vetorial $V$ sob representação de $\Gamma$ em $V$.

Quando não houver dúvidas quanto usarmos a ação ou a representação de $\Gamma$ em $V$, escrevemos $\gamma x$ ou $\rho(\gamma) x$ indistintamente. 
Agora, apresentamos a integral de Haar que é uma ferramenta importante para a teoria de representação e é usada na Seção 1.5 para as fórmulas de Molien e também no Capítulo 3.

A integral de Haar é definida de forma abstrata como uma operação que satisfaz três propriedades. A princípio, ela é definida para grupos localmente compactos, mas aqui nós restringimos a definição para grupos de Lie compactos. Definimos para funções contínuas $\Gamma \rightarrow \mathbb{R}$, e o conceito é estendido naturalmente para aplicações contínuas $\Gamma \rightarrow \mathbb{R}^{n}$.

Definição 1.1.1. Seja $f: \Gamma \rightarrow \mathbb{R}$ uma função contínua e $\Gamma$ um grupo de Lie compacto. A operação $\int_{\Gamma} f d \gamma$ é uma integral de Haar em $\Gamma$ se satizfaz:

1) Linearidade: $\int_{\Gamma}(a f+b g) d \gamma=a \int_{\Gamma} f d \gamma+b \int_{\Gamma} g d \gamma$ para $f, g: \Gamma \rightarrow \mathbb{R}$ contínuas $e$ $a, b \in \mathbb{R} ;$

2) Positividade: Se $f(\gamma) \geqslant 0$, para todo $\gamma \in \Gamma$, então $\int_{\Gamma} f d \gamma \geqslant 0$;

3) Invariância por translação: $\int_{\Gamma} f(\tau \gamma) d \gamma=\int_{\Gamma} f(\gamma) d \gamma$, para todo $\tau \in \Gamma$ fixado.

A prova da existência e unicidade da integral de Haar pode ser encontrada em [36, 52].

Como $\Gamma$ é compacto, a integral de Haar também é invariante pela esquerda:

$$
\int_{\Gamma} f(\gamma \tau) d \gamma=\int_{\Gamma} f(\gamma) d \gamma, \forall \tau \in \Gamma
$$

Além disso, neste caso $\int_{\Gamma} 1 d \gamma$ é finito e, portanto, podemos considerar a integral de Haar normalizada: $\int_{\Gamma} 1 d \gamma=1$. Se $\Gamma$ é finito de ordem $|\Gamma|$, então a integral de Haar normalizada em $\Gamma$ é dada por

$$
\int_{\Gamma} f d \gamma \equiv \frac{1}{|\Gamma|} \sum_{\gamma \in \Gamma} f(\gamma) .
$$

Através de um produto interno qualquer em $V$ e da integral de Haar, construímos um produto interno $\langle,\rangle_{\Gamma} \Gamma$-invariante em $V$ ([35], Capítulo XII, Proposição 1.3), que satisfaz

$$
\langle\gamma v, \gamma w\rangle_{\Gamma}=\langle v, w\rangle_{\Gamma}, \forall \gamma \in \Gamma, \quad \forall v, w \in V
$$

Então, sem perda de generalidade assumimos que a ação de $\Gamma$ em $V$ é ortogonal. Logo, $\Gamma$ pode ser identificado como um subgrupo fechado do grupo ortogonal $\mathbf{O}(n)$ onde $n$ é a dimensão de $V$. 


\subsection{Invariantes e equivariantes}

Neste trabalho usamos a linguagem da teoria de singularidades para tratar do estudo local de aplicações $C^{\infty}$ na vizinhança de um ponto, o qual assumimos ser a origem, através da noção de germes de aplicações (veja Gibson [33]). Denotamos estes germes por $g: V, 0 \rightarrow W$ para espaços vetoriais reais $V$ e $W$, ambos de dimensão finita.

Denotamos por $\mathcal{E}_{V}$ o anel de germes de funções $V, 0 \rightarrow \mathbb{R}$ e por $\overrightarrow{\mathcal{E}}_{V, W}$ o módulo dos germes de aplicações $V, 0 \rightarrow W$ sobre o anel $\mathcal{E}_{V}$.

No restante deste capítulo, sejam $(\rho, V)$ e $(\eta, W)$ representações de $\Gamma$ em $V$ e $W$, respectivamente.

Um germe de função $f: V, 0 \rightarrow \mathbb{R}$ é chamado de $\Gamma$-invariante se

$$
f(\rho(\gamma) x)=f(x), \quad \forall \gamma \in \Gamma, \quad \forall x \in V, 0 .
$$

Denotamos por $\mathcal{P}_{V}(\Gamma)$ o anel dos germes de polinômios $\Gamma$-invariantes e por $\mathcal{E}_{V}(\Gamma)$ o anel dos germes de funções $C^{\infty} \Gamma$-invariantes.

Um germe de aplicação $g: V, 0 \rightarrow W$ é chamado de $\Gamma$-equivariante se

$$
g(\rho(\gamma) x)=\eta(\gamma) g(x), \quad \forall \gamma \in \Gamma, \quad \forall x \in V, 0
$$

Denotamos por $\overrightarrow{\mathcal{P}}_{V, W}(\Gamma)$ os germes de aplicações polinomiais $\Gamma$-equivariantes e por $\overrightarrow{\mathcal{E}}_{V, W}(\Gamma)$ os germes de aplicações $C^{\infty} \Gamma$-equivariantes. Quando $V=W$ escrevemos apenas $\overrightarrow{\mathcal{P}}_{V}(\Gamma)$ e $\overrightarrow{\mathcal{E}}_{V}(\Gamma)$.

O espaço $\mathcal{P}_{V}(\Gamma)$ tem estrutura de anel e, sendo $\Gamma$ compacto, $\mathcal{P}_{V}(\Gamma)$ possui um conjunto finito de geradores, ou seja, existe um número finito de funções polinomiais $\Gamma$-invariantes, $u_{1}, \ldots, u_{s}$, tal que todo polinômio em $\mathcal{P}_{V}(\Gamma)$ pode ser escrito como função de $u_{1}, \ldots, u_{s}$. Este conjunto não é único e é chamado base de Hilbert (veja [35, Capítulo XII, Teorema $4.2])$ :

Teorema 1.2.1. Seja $\Gamma$ um grupo de Lie compacto agindo em V. Então existe uma base de Hilbert finita $u_{1}, \ldots, u_{s}$ para o anel $\mathcal{P}_{V}(\Gamma)$.

Além disso, $\mathcal{P}_{V}(\Gamma)$ é um subanel de $\mathcal{E}_{V}(\Gamma)$ e ambos possuem a mesma base de Hilbert. Este resultado é devido a Schwarz (veja [35, Capítulo XII, Teorema 4.3]): 
Teorema 1.2.2. (Teorema de Schwarz) Seja $\Gamma$ um grupo de Lie compacto agindo em $V$ e seja $u_{1}, \ldots, u_{s}$ uma base de Hilbert para $\mathcal{P}_{V}(\Gamma)$. Se $f \in \mathcal{E}_{V}(\Gamma)$, então existe um germe suave $h: \mathbb{R}^{s}, 0 \rightarrow \mathbb{R}$ tal que

$$
f(x)=h\left(u_{1}(x), \ldots, u_{s}(x)\right)
$$

Se $f$ é $\Gamma$-invariante e $g$ é $\Gamma$-equivariante, então $f g: V, 0 \rightarrow W$ é $\Gamma$-equivariante. Logo, $\overrightarrow{\mathcal{P}}_{V, W}(\Gamma)$ e $\overrightarrow{\mathcal{E}}_{V, W}(\Gamma)$ têm estrutura de módulos sobre os anéis $\mathcal{P}_{V}(\Gamma)$ e $\mathcal{E}_{V}(\Gamma)$, respectivamente.

Dizemos que as aplicações $g_{1}, \ldots, g_{r} \Gamma$-equivariantes geram o módulo $\overrightarrow{\mathcal{P}}_{V, W}(\Gamma)$ sobre o anel $\mathcal{P}_{V}(\Gamma)$ se toda aplicação $g \in \overrightarrow{\mathcal{P}}_{V, W}(\Gamma)$ pode ser escrita como

$$
g=f_{1} g_{1}+\ldots+f_{m} g_{m}
$$

para funções $f_{1}, \ldots, f_{m} \Gamma$-invariantes. Neste caso, se $g_{1}, \ldots, g_{r}$ geram $\overrightarrow{\mathcal{P}}_{V, W}(\Gamma)$ usamos a notação:

$$
\overrightarrow{\mathcal{P}}_{V, W}(\Gamma)=\mathcal{P}_{V}(\Gamma)\left\{g_{1}, \ldots, g_{m}\right\}
$$

A seguir enunciamos o Teorema de Poènaru [35, Capítulo XII, Teoremas 5.2 e 5.3] que relaciona os conjuntos geradores para os módulos $\overrightarrow{\mathcal{P}}_{V, W}(\Gamma)$ e $\overrightarrow{\mathcal{E}}_{V, W}(\Gamma)$ :

Teorema 1.2.3. (Teorema de Poènaru) Seja $\Gamma$ um grupo de Lie compacto agindo em $V$ e $W$.

1) O módulo $\overrightarrow{\mathcal{P}}_{V, W}(\Gamma)$ é finitamente gerado sobre o anel $\mathcal{P}_{V}(\Gamma)$;

2) Sejam $g_{1}, \ldots, g_{r}$ geradores para o módulo $\overrightarrow{\mathcal{P}}_{V, W}(\Gamma)$ sobre o anel $\mathcal{P}_{V}(\Gamma)$. Então $g_{1}, \ldots, g_{r}$ também geram o módulo $\overrightarrow{\mathcal{E}}_{V, W}(\Gamma)$ sobre o anel $\mathcal{E}_{V}(\Gamma)$.

\subsection{O contexto com simetrias e antissimetrias}

Formalizamos esta teoria através de ferramentas algébricas. Assim, seja $(\rho, V)$ representação de um grupo de Lie compacto $\Gamma$. O ponto de partida é considerar o homomorfismo

$$
\sigma: \Gamma \rightarrow \mathbf{Z}_{2}
$$

definido como: $\gamma$ é uma simetria se $\sigma(\gamma)=1$ e $\gamma$ é uma antissimetria se $\sigma(\gamma)=-1$. Aqui, $\mathbf{Z}_{2}$ denota o grupo multiplicativo $\{1,-1\}$. 
Sob este formalismo, denotamos por $\Gamma_{+}$o subgrupo das simetrias de $\Gamma$, o qual é um subgrupo normal de índice 2 de $\Gamma$, e por $\Gamma_{-}$o conjunto das antissimetrias de $\Gamma$. Como consequência direta notamos que o produto entre duas simetrias e entre duas antissimetrias é uma simetria, enquanto que o produto entre uma simetria e uma antissimetria é uma antissimetria.

Para uma antissimetria arbitrária fixada $\delta$, temos $\Gamma_{-}=\delta \Gamma_{+}$e

$$
\Gamma=\Gamma_{+} \dot{\cup} \delta \Gamma_{+}
$$

Ressaltamos que aqui neste trabalho sempre que $\delta$ aparecer estará denotando uma antissimetria fixada do grupo $\Gamma$.

Um germe de função $f: V, 0 \rightarrow \mathbb{R}$ é chamado de $\Gamma$-anti-invariante se

$$
f(\rho(\gamma) x)=\sigma(\gamma) f(x), \quad \forall \gamma \in \Gamma, \quad \forall x \in V, 0 .
$$

Denotamos por $\mathcal{Q}_{V}(\Gamma)$ o módulo dos germes de funções polinomiais $\Gamma$-anti-invariantes e por $\mathcal{F}_{V}(\Gamma)$ o módulo dos germes de funções $C^{\infty} \Gamma$-anti-invariantes.

Um germe de aplicação $g: V, 0 \rightarrow V$ é chamado de $\Gamma$-reversível-equivariante se

$$
g(\rho(\gamma) x)=\sigma(\gamma) \rho(\gamma) g(x), \quad \forall \gamma \in \Gamma, \quad \forall x \in V, 0 .
$$

Denotamos por $\overrightarrow{\mathcal{Q}}_{V}(\Gamma)$ o módulo dos germes de aplicações polinomiais $\Gamma$-reversíveisequivariantes e por $\overrightarrow{\mathcal{F}}_{V}(\Gamma)$ o módulo dos germes de aplicações $C^{\infty} \Gamma$-reversíveis-equivariantes.

Note que a composição de uma aplicação $\Gamma$-reversível-equivariante com uma aplicação $\Gamma$-equivariante é $\Gamma$-reversível-equivariante.

A partir do homomorfismo $\sigma$ de (1.4) e da representação $(\rho, V)$ definimos a representação $\sigma$-dual de $\rho$ como:

$$
\begin{aligned}
\rho_{\sigma}: \Gamma & \rightarrow \mathbf{G L}(V) \\
\gamma & \mapsto \sigma(\gamma) \rho(\gamma) .
\end{aligned}
$$

A ação correspondente à representação dual é chamada de ação dual. Note que $\left(\rho_{\sigma}\right)_{\sigma}=$ $\rho$.

Duas representações $(\rho, V)$ e $(\eta, W)$ de $\Gamma$ são $\Gamma$-isomorfas se existe um isomorfismo linear $A:(\rho, V) \rightarrow(\eta, W) \Gamma$-equivariante, ou seja, que satisfaz

$$
A(\rho(\gamma) x)=\eta(\gamma) A(x), \quad \forall \gamma \in \Gamma, \quad \forall x \in V .
$$


Também dizemos que os espaços vetoriais $V$ e $W$ são $\Gamma$-isomorfos. Se as representações $(\rho, V)$ e $\left(\rho_{\sigma}, V\right)$ são $\Gamma$-isomorfas, dizemos que a representação $\rho$ é auto-dual.

Considerando a representação dual (1.8), reescrevemos $g(\rho(\gamma) x)=\rho_{\sigma}(\gamma) g(x)$ e percebemos que um germe de aplicação de $(\rho, V)$ em $(\rho, V)$-reversível-equivariante é um germe aplicação $\Gamma$-equivariante de $(\rho, V)$ em $\left(\rho_{\sigma}, V\right)$. Analogamente, o germe de função $\Gamma$-anti-invariante $f: V, 0 \rightarrow \mathbb{R}$ é um germe de aplicação $\Gamma$-equivariante de $(\rho, V)$ em $(\sigma, \mathbb{R})$. Portanto, pelo Teorema de Poènaru 1.2 .3 , segue que $\mathcal{Q}_{V}(\Gamma)$ e $\overrightarrow{\mathcal{Q}}_{V}(\Gamma)$ são módulos finitamente gerados sobre o anel $\mathcal{P}_{V}(\Gamma)$.

Terminamos esta seção apresentando o Teorema de Fubini ([8, Proposição 5.16]), o qual reduz o cálculo da integral de Haar sobre $\Gamma$ para integrais sobre $\Gamma_{+}$:

Teorema 1.3.1. Seja $\Gamma$ um grupo de Lie compacto $e \Gamma_{+} \subset \Gamma$ um subgrupo de indice 2 em $\Gamma$. Para qualquer $f: \Gamma \rightarrow \mathbb{R}$ contínua temos

$$
\int_{\Gamma} f(\gamma)=\frac{1}{2}\left(\int_{\Gamma_{+}} f(\gamma)+\int_{\Gamma_{+}} f(\delta \gamma)\right)
$$

para $\delta \in \Gamma_{-}$fixo.

No Capítulo 3 o Teorema de Fubini é usado na demonstração do teorema principal.

\subsection{Operadores de Reynolds e algoritmos}

Considere o espaço $\mathcal{P}_{V}$ dos germes de funções polinomiais homogêneas $V, 0 \rightarrow \mathbb{R}$ e o espaço $\overrightarrow{\mathcal{P}}_{V}$ dos germes de aplicações polinomiais homogêneas $V, 0 \rightarrow V$. Definimos ações de $\Gamma$ nestes espaços induzidas da ação de $\Gamma$ em $V$ :

$$
\begin{array}{rlrl}
\Gamma \times \mathcal{P}_{V} & \rightarrow \mathcal{P}_{V} & \text { e } & \Gamma \times \overrightarrow{\mathcal{P}}_{V} \rightarrow \overrightarrow{\mathcal{P}}_{V}, \\
(\gamma, f) \mapsto \gamma \odot f & & (\gamma, g) \mapsto \gamma \star g
\end{array}
$$

onde $\gamma \odot f(x)=f(\gamma x) \quad$ e $\quad \gamma \star g(x)=\gamma^{-1} g(\gamma x), \quad \forall \gamma \in \Gamma, \quad \forall x \in V, 0,$.

Consideramos os operadores de Reynolds em $\mathcal{P}_{V}\left(\Gamma_{+}\right)$, a saber, $R, S: \mathcal{P}_{V}\left(\Gamma_{+}\right) \rightarrow \mathcal{P}_{V}\left(\Gamma_{+}\right)$ definidos por

$$
\begin{gathered}
R(f)=\frac{1}{2} \sum_{\gamma \Gamma_{+}} \gamma \odot f=\frac{1}{2}(f+\delta \odot f), \\
S(f)=\frac{1}{2} \sum_{\gamma \Gamma_{+}} \sigma(\gamma) \gamma \odot f=\frac{1}{2}(f-\delta \odot f) .
\end{gathered}
$$


De forma análoga existe a versão equivariante para os operadores $R$ e $S$. Considere $\vec{R}, \vec{S}: \overrightarrow{\mathcal{P}}_{V}\left(\Gamma_{+}\right) \rightarrow \overrightarrow{\mathcal{P}}_{V}\left(\Gamma_{+}\right)$, definidos por

$$
\begin{gathered}
\vec{R}(g)=\frac{1}{2} \sum_{\gamma \Gamma_{+}} \gamma \star g=\frac{1}{2}(g+\delta \star g), \\
\vec{S}(g)=\frac{1}{2} \sum_{\gamma \Gamma_{+}} \sigma(\gamma) \gamma \star g=\frac{1}{2}(g-\delta \star g) .
\end{gathered}
$$

Em [2], os operadores de Reynolds são usados para provar as decomposições de $\mathcal{P}_{V}(\Gamma)$ - módulos

$$
\mathcal{P}_{V}\left(\Gamma_{+}\right)=\mathcal{P}_{V}(\Gamma) \oplus \mathcal{Q}_{V}(\Gamma) \quad \text { e } \quad \overrightarrow{\mathcal{P}}_{V}\left(\Gamma_{+}\right)=\overrightarrow{\mathcal{P}}_{V}(\Gamma) \oplus \overrightarrow{\mathcal{Q}}_{V}(\Gamma)
$$

as quais são usadas para obter o algoritmo que determina geradores para $\mathcal{Q}_{V}(\Gamma)$ e $\overrightarrow{\mathcal{Q}}_{V}(\Gamma)$ a partir dos geradores de $\mathcal{P}_{V}\left(\Gamma_{+}\right)$e $\overrightarrow{\mathcal{P}}_{V}\left(\Gamma_{+}\right)$. Além disso, os operadores $R, S, \vec{R}$ e $\vec{S}$ satisfazem as condições a seguir (veja [2]):

(1) São homomorfismos entre $\mathcal{P}_{V}(\Gamma)$-módulos, com

$$
R_{\Gamma_{+}}^{\Gamma}+S_{\Gamma_{+}}^{\Gamma}=I_{\mathcal{P}_{V}\left(\Gamma_{+}\right)} \quad \text { e } \quad \vec{R}_{\Gamma_{+}}^{\Gamma}+\vec{S}_{\Gamma_{+}}^{\Gamma}=I_{\overrightarrow{\mathcal{P}}_{V}\left(\Gamma_{+}\right)},
$$

onde $I_{\mathcal{P}_{V}\left(\Gamma_{+}\right)}$e $I_{\overrightarrow{\mathcal{P}}_{V}\left(\Gamma_{+}\right)}$são as identidades em $\mathcal{P}_{V}\left(\Gamma_{+}\right)$e $\overrightarrow{\mathcal{P}}_{V}\left(\Gamma_{+}\right)$, respectivamente.

(2) São projeções idempotentes:

$$
\begin{array}{lll}
\operatorname{ker} R_{\Gamma_{+}}^{\Gamma}=\operatorname{Im} S_{\Gamma_{+}}^{\Gamma}=\mathcal{Q}_{V}(\Gamma) & \text { e } & \operatorname{ker} S_{\Gamma_{+}}^{\Gamma}=\operatorname{Im} R_{\Gamma_{+}}^{\Gamma}=\mathcal{P}_{V}(\Gamma) \\
\operatorname{ker} \vec{R}_{\Gamma_{+}}^{\Gamma}=\operatorname{Im} \vec{S}_{\Gamma_{+}}^{\Gamma}=\overrightarrow{\mathcal{Q}}_{V}(\Gamma) & \text { e } & \operatorname{ker} \vec{S}_{\Gamma_{+}}^{\Gamma}=\operatorname{Im} \vec{R}_{\Gamma_{+}}^{\Gamma}=\overrightarrow{\mathcal{P}}_{V}(\Gamma)
\end{array}
$$

A partir dos operadores de Reynolds é possível construir um conjunto finito de geradores para o módulo $\overrightarrow{\mathcal{Q}}_{V}(\Gamma)$ sobre o anel $\mathcal{P}_{V}(\Gamma)$ fixando uma antissimetria arbitrária $\delta$, e conhecendo apenas os geradores para $\mathcal{P}_{V}\left(\Gamma_{+}\right)$e $\overrightarrow{\mathcal{P}}_{V}\left(\Gamma_{+}\right)$. Este processo pode facilitar de forma considerável o estudo de sistemas reversíveis equivariantes, uma vez que conhecendo o grupo $\Gamma_{+}$é possível determinar uma forma geral do sistema através do algoritmo em [2]:

\section{Algoritmo 1.4.1. (Geradores para $\mathcal{Q}_{V}(\Gamma)$ e $\overrightarrow{\mathcal{Q}}_{V}(\Gamma)$ )}

1. Considere um grupo de Lie compacto $\Gamma$, defina um subgrupo normal $\Gamma_{+} \subset \Gamma$ de indice 2 e fixe uma anti-simetria $\delta \in \Gamma_{-}$; 
2. Tome uma base de Hilbert homogênea $\left\{u_{1}, \ldots, u_{s}\right\}$ para $\mathcal{P}_{V}\left(\Gamma_{+}\right)$;

3. Determine um conjunto $\left\{H_{0}, \ldots, H_{r}\right\}$ de geradores homogêneos para $\overrightarrow{\mathcal{P}}_{V}\left(\Gamma_{+}\right)$como módulo sobre $\mathcal{P}_{V}\left(\Gamma_{+}\right)$.

4. Defina $\tilde{u}_{0}=1$. Para $i=1$ até s faça

$$
\tilde{u}_{i}=S\left(u_{i}\right)
$$

5. Para $j=0$ até $r$ e para $i=0$ até s faça

$$
\begin{aligned}
H_{0 j} & =H_{j} ; \\
H_{i j} & =\tilde{u}_{i}(x) H_{j} ; \\
\tilde{H}_{i j} & =\vec{S}\left(H_{i j}\right) .
\end{aligned}
$$

Resultado: Obtenção de um conjunto gerador homogêneo $\left\{\tilde{u}_{1}, \ldots, \tilde{u}_{s}\right\}$ para o módulo $\mathcal{Q}_{V}(\Gamma)$ e de um conjunto gerador homogêneo $\left\{\tilde{H}_{00}, \ldots, \tilde{H}_{s r}\right\}$ para o módulo $\overrightarrow{\mathcal{Q}}_{V}(\Gamma)$, ambos sobre o anel $\mathcal{P}_{V}(\Gamma)$.

Após aplicar o Algoritmo 1.4.1 é necessário determinar uma base de Hilbert para o anel $\mathcal{P}_{V}(\Gamma)$. A obtenção desta base pode ser feita a partir da base de Hilbert de $\mathcal{P}_{V}\left(\Gamma_{+}\right)$ como enunciado no teorema apresentado em [4]:

Teorema 1.4.2. Seja $\Gamma$ um grupo de Lie compacto agindo linearmente em $V$ e seja $\sigma: \Gamma \rightarrow Z_{2}$ o homomorfismo definido em (1.4), com $\Gamma_{+}=\operatorname{ker} \sigma$. Seja $u_{1}, \ldots, u_{s}$ uma base de Hilbert para o anel $\mathcal{P}_{V}\left(\Gamma_{+}\right)$. Então, o conjunto

$$
\left\{R\left(u_{i}\right), S\left(u_{i}\right) S\left(u_{j}\right), \text { para } 1 \leq i, j \leq s\right\}
$$

é uma base de Hilbert para o anel $\mathcal{P}_{V}(\Gamma)$.

A ideia básica do Algoritmo 1.4.1 é facilitar o cálculo da forma geral $\Gamma$-reversívelequivariante a partir do conhecimento de geradores para $\mathcal{P}_{V}\left(\Gamma_{+}\right)$e $\overrightarrow{\mathcal{P}}_{V}\left(\Gamma_{+}\right)$. Mas o processo pode ser ainda mais reduzido. Em [35, Teorema 6.8] é apresentado um processo que permite encontrar geradores para $\overrightarrow{\mathcal{P}}_{V}\left(\Gamma_{+}\right)$através de uma base de Hilbert por uma ação particular de $\Gamma_{+}$em $V \times V$. Apresentamos brevemente este resultado. 
Considere a ação de $\Gamma_{+}$em $V$ e defina a ação de $\Gamma_{+}$em $V \times V, \gamma(x, y)=(\gamma x, \gamma y)$. Seja $u_{1}, \ldots, u_{s}$ uma base de Hilbert para o anel $\mathcal{P}_{V \times V}\left(\Gamma_{+}\right)$e seja

$$
H_{i}(x)=\left(d u_{i}\right)_{y}(x, 0)
$$

onde $(x, y) \in V, 0 \times V, 0$ e $\left(d u_{i}\right)_{y}$ é a derivada parcial de $u_{i}$ em relação a $y$ para $1 \leq i \leq s$. Então, as aplicações $H_{1}, \ldots, H_{s}$ geram o módulo $\overrightarrow{\mathcal{P}}_{V}\left(\Gamma_{+}\right)$sobre o anel $\mathcal{P}_{V}\left(\Gamma_{+}\right)$.

Enfatizamos que sempre que $\Gamma_{+}$for um grupo de Lie compacto finitamente gerado, as bases de Hilbert para $\mathcal{P}_{V}\left(\Gamma_{+}\right)$e $\mathcal{P}_{V \times V}\left(\Gamma_{+}\right)$podem ser obtidas com o programa Singular [55], separadamente, a partir apenas da representação matricial dos geradores de $\Gamma_{+}$.

Observação 1.4.3. Embora a teoria seja feita para grupos de Lie compactos, o Algoritmo 1.4 .1 e o Teorema 1.4.2 podem ser aplicados para grupos não compactos $\Gamma$ desde que haja uma base de Hilbert (finita) para os invariantes e um conjunto gerador finito para os equivariantes segundo a ação de $\Gamma_{+}$.

\subsection{Séries de Hilbert e fórmulas de Molien}

Nesta seção apresentamos resultados relacionados à teoria invariante para as séries de Hilbert de $\mathcal{P}_{V}(\Gamma), \overrightarrow{\mathcal{P}}_{V, W}(\Gamma), \mathcal{Q}_{V}(\Gamma)$ e $\overrightarrow{\mathcal{Q}}_{V}(\Gamma)$. Estas séries são úteis para verificar se o número dos geradores destes espaços em cada grau foram obtidos.

Em [2], os autores obtêm fórmulas para as séries de Hilbert nos casos anti-invariantes e reversíveis equivariantes usando teoria de caracter. A vantagem é que o caracter de uma representação é único e, além disso, o cálculo com caracteres é padrão em alguns pacotes de álgebra computacional, como o GAP [32]. Neste programa há uma função para calcular as séries de Hilbert dos invariantes e dos equivariantes, sendo necessário fornecer apenas os geradores do grupo e o caracter da representação.

Sejam $(\rho, V)$ e $(\eta, W)$ representações de $\Gamma$. Como o anel $\mathcal{P}_{V}$ é uma álgebra graduada sobre $\mathbb{R}$, ou seja,

$$
\mathcal{P}_{V}=\bigoplus_{k=0}^{\infty} \mathcal{P}_{V}^{k}
$$

onde $\mathcal{P}_{V}^{k}$ é o espaço dos germes de polinômios homogêneos $V, 0 \rightarrow V$ de grau $k$. Como a ação de $\Gamma$ em $V$ é linear, se $f \in \mathcal{P}_{V}^{k}$ para algum $k$, então $\gamma \odot f \in \mathcal{P}_{V}^{k}, \forall \gamma \in \Gamma, \forall x \in V, 0$. 
Assim, o anel $\mathcal{P}_{V}(\Gamma)$ tem a estrutura de uma álgebra graduada, dada por

$$
\mathcal{P}_{V}(\Gamma)=\bigoplus_{k=0}^{\infty} \mathcal{P}_{V}^{k}(\Gamma)
$$

onde $\mathcal{P}_{V}^{k}(\Gamma)=\mathcal{P}_{V}(\Gamma) \cap \mathcal{P}_{V}^{k}$. Analogamente, seja $\overrightarrow{\mathcal{P}}_{V, W}$ o espaço dos germes de aplicações polinomiais $V, 0 \rightarrow W$. Então, $\overrightarrow{\mathcal{P}}_{V, W}$ tem a estrutura de um módulo graduado sobre o anel $\mathcal{P}_{V}$

$$
\overrightarrow{\mathcal{P}}_{V, W}=\bigoplus_{k=0}^{\infty} \overrightarrow{\mathcal{P}}_{V, W}^{k}
$$

onde $\overrightarrow{\mathcal{P}}_{V, W}^{k}$ é o espaço dos germes de aplicações polinomiais homogêneas de grau $k$. Naturalmente temos

$$
\overrightarrow{\mathcal{P}}_{V, W}(\Gamma)=\bigoplus_{k=0}^{\infty} \overrightarrow{\mathcal{P}}_{V, W}^{k}(\Gamma)
$$

$\operatorname{com} \overrightarrow{\mathcal{P}}_{V, W}^{k}(\Gamma)=\overrightarrow{\mathcal{P}}_{V, W}(\Gamma) \cap \overrightarrow{\mathcal{P}}_{V, W}^{k}$

A série de Hilbert para o anel $\mathcal{P}_{V}(\Gamma)$ é uma função geradora para a dimensão do espaço vetorial dos polinômios invariantes homogêneos de cada grau e é definida pela série formal

$$
\Phi_{V}^{\Gamma}(t)=\sum_{k=0}^{\infty} \operatorname{dim} \mathcal{P}_{V}^{k}(\Gamma) t^{k}
$$

De forma análoga, a série de Hilbert para $\overrightarrow{\mathcal{P}}_{V, W}(\Gamma)$ é uma função geradora para a dimensão dos espaços vetoriais dos polinômios homogêneos equivariantes de cada grau e é definida pela série formal

$$
\Psi_{V}^{\Gamma}(t)=\sum_{k=0}^{\infty} \operatorname{dim} \overrightarrow{\mathcal{P}}_{V, W}^{k}(\Gamma) t^{k}
$$

Temos ainda

$$
\mathcal{Q}_{V}(\Gamma)=\bigoplus_{k=0}^{\infty} \mathcal{Q}_{V}^{k}(\Gamma) \quad \text { e } \quad \overrightarrow{\mathcal{Q}}_{V}(\Gamma)=\bigoplus_{k=0}^{\infty} \overrightarrow{\mathcal{Q}}_{V}^{k}(\Gamma)
$$

onde $\mathcal{Q}_{V}^{k}(\Gamma)=\mathcal{Q}_{V}(\Gamma) \cap \mathcal{P}_{V}^{k}$ e $\overrightarrow{\mathcal{Q}}_{V}^{k}(\Gamma)=\overrightarrow{\mathcal{Q}}_{V}(\Gamma) \cap \overrightarrow{\mathcal{P}}_{V}^{k}$. As séries de Hilbert para os módulos $\mathcal{Q}_{V}(\Gamma)$ e $\overrightarrow{\mathcal{Q}}_{V}(\Gamma)$, definidas em [2], são dadas por:

$$
\tilde{\Phi}_{V}^{\Gamma}(t)=\sum_{k=0}^{\infty} \operatorname{dim} \mathcal{Q}_{V}^{k}(\Gamma) t^{k} \quad \text { e } \quad \tilde{\Psi}_{V}^{\Gamma}(t)=\sum_{k=0}^{\infty} \operatorname{dim} \overrightarrow{\mathcal{Q}}_{V}^{k}(\Gamma) t^{k} .
$$

Calcular as séries de Hilbert manualmente pode ser uma trabalho árduo. Mas estas séries têm fórmulas explícitas que são dadas em termos da integral de Haar normalizada sobre $\Gamma$. Os próximos dois teoremas nos fornecem este resultado. 
Teorema 1.5.1. (Teorema de Molien) Seja $\Gamma$ um grupo de Lie compacto. Então a série de Hilbert de $\mathcal{P}_{V}(\Gamma)$ é dada por

$$
\Phi_{V}^{\Gamma}(t)=\int_{\Gamma} \frac{1}{\operatorname{det}(1-t \rho(\gamma))}
$$

Veja [56] para a prova no caso finito e [54] para a extensão a um grupo compacto.

A generalização do Teorema de Molien para o caso equivariante é dado em [54]. Primeiramente, lembramos que o caracter $\chi_{V}$ de uma representação $\rho: \Gamma \rightarrow \mathbf{G L}(V)$ é definido como

$$
\chi_{V}: \Gamma \rightarrow \mathbb{R}
$$

onde $\chi_{V}(\gamma)$ é o traço da matriz $\rho(\gamma) \in \mathbf{G L}(V)$.

Observamos que representações $\Gamma$-isomorfas têm o mesmo caracter. Além disso, como a representação de $\Gamma$ é ortogonal, tem-se $\chi_{V}\left(\gamma^{-1}\right)=\chi_{V}(\gamma)$.

Teorema 1.5.2. (Teorema de Molien equivariante) Seja $\Gamma$ um grupo de Lie compacto. Então a série de Hilbert de $\overrightarrow{\mathcal{P}}_{V}(\Gamma)$ é dada por

$$
\Psi_{V}^{\Gamma}(t)=\int_{\Gamma} \frac{\chi_{V}\left(\gamma^{-1}\right)}{\operatorname{det}(1-t \rho(\gamma))}
$$

onde $\chi_{V}$ é o caracter da representação $(\rho, V)$.

Em [61] é feita a generalização do Teorema 1.5.2 para aplicações equivariantes $g$ : $(\rho, V) \rightarrow(\eta, W)$, sendo $\rho$ e $\eta$ representações distintas, ou seja, não $\Gamma$-isomorfas. Neste caso, a série de Hilbert de $\overrightarrow{\mathcal{P}}_{V, W}(\Gamma)$ é dada por

$$
\Psi_{V, W}^{\Gamma}(t)=\int_{\Gamma} \frac{\chi_{W}\left(\gamma^{-1}\right)}{\operatorname{det}(1-t \rho(\gamma))}
$$

onde $\chi_{W}$ é o caracter da representação $(\eta, W)$.

Como já falamos anteriormente, os casos anti-invariantes e reversíveis equivariantes se reduzem ao contexto equivariante. Assim, de (1.20) segue de imediato as fórmulas de Molien para as séries de Hilbert dos módulos $\mathcal{Q}_{V}(\Gamma)$ e $\overrightarrow{\mathcal{Q}}_{V}(\Gamma)$ (veja [2]):

$$
\tilde{\Phi}_{V}^{\Gamma}(t)=\int_{\Gamma} \frac{\sigma(\gamma)}{\operatorname{det}(1-t \rho(\gamma))} \quad \text { e } \quad \tilde{\Psi}_{V}^{\Gamma}(t)=\int_{\Gamma} \frac{\sigma(\gamma) \chi_{V}(\gamma)}{\operatorname{det}(1-t \rho(\gamma))}
$$

Na Seção 2.3 tratamos estas séries para os invariantes e equivariantes no caso particular da ação diagonal de dois grupos. 


$\frac{1}{2}$

\section{Teoria invariante para o produto} semi-direto

Neste capítulo relembramos conceitos sobre o produto semi-direto de dois grupos e introduzimos uma linguagem apropriada para a teoria invariante deste produto. As ferramentas desenvolvidas aqui são usadas no Capítulo 3 e, principalmente, aplicadas no Capítulo 5 para uma classificação de formas normais reversíveis equivariantes. Quando o grupo de simetrias e antissimetrias de um sistema é um produto semi-direto percebemos que a teoria reversível equivariante do produto está diretamente relacionada à teoria de cada grupo, separadamente.

Na Seção 2.1 formalizamos a teoria de representação do produto semi-direto e a teoria reversível equivariante para tal grupo. Na Seção 2.2 apresentamos a teoria invariante para a representação diagonal e resultados sobre a obtenção de uma base de Hilbert para os invariantes e um conjunto gerador para os equivariantes segundo a ação do produto semidireto. A Seção 2.3 é dedicada à obtenção das fórmulas de Molien para os invariantes e equivariantes sob a ação diagonal. 


\subsection{Invariantes para o produto semi-direto}

Dados dois grupos $\Gamma_{1}$ e $\Gamma_{2}$, lembremos que o produto semi-direto $\Gamma_{1} \rtimes \Gamma_{2}$ é o produto direto $\Gamma_{1} \times \Gamma_{2}$ como conjunto munido de uma operação induzida por um homomorfismo

$$
\mu: \Gamma_{2} \rightarrow \operatorname{Aut}\left(\Gamma_{1}\right)
$$

onde

$$
\left(\gamma_{1}, \gamma_{2}\right)_{\cdot \mu}\left(\tau_{1}, \tau_{2}\right)=\left(\gamma_{1} \mu\left(\gamma_{2}\right) \tau_{1}, \gamma_{2} \tau_{2}\right)
$$

Por construção, $\Gamma_{1}$ é um subgrupo normal de $\Gamma_{1} \rtimes \Gamma_{2}$.

Sejam $(\rho, V)$ e $(\eta, V)$ representações de $\Gamma_{1}$ e $\Gamma_{2}$ em um espaço vetorial real de dimensão finita $V$, respectivamente, e considere a operação $\left(\Gamma_{1} \rtimes \Gamma_{2}\right) \times V \rightarrow V$ :

$$
\left(\gamma_{1}, \gamma_{2}\right) v=\gamma_{1}\left(\gamma_{2} v\right)
$$

Apresentamos abaixo nosso primeiro resultado relativo a ações de produtos semidiretos:

Proposição 2.1.1. A operação (2.2) define uma ação do produto semi-direto $\Gamma_{1} \rtimes \Gamma_{2}$ em $V$ se, e somente se, a representação de $\mu\left(\gamma_{2}\right)\left(\gamma_{1}\right)$ é uma conjugação, isto é, $\rho\left(\mu\left(\gamma_{2}\right)\left(\gamma_{1}\right)\right)=$ $\eta\left(\gamma_{2}\right) \rho\left(\gamma_{1}\right) \eta\left(\gamma_{2}\right)^{-1}$.

Demonstração: Segue da definição de ação de grupo que $(2.2)$ define uma ação se, e somente se,

$$
\left(\gamma_{1}, \gamma_{2}\right)\left(\left(\tau_{1}, \tau_{2}\right) v\right)=\left(\left(\gamma_{1}, \gamma_{2}\right) \cdot \mu\left(\tau_{1}, \tau_{2}\right)\right) v
$$

para $\gamma_{1}, \tau_{1} \in \Gamma_{1}, \quad \gamma_{2}, \tau_{2} \in \Gamma_{2}$ e $v \in V$. Da Definição 2.2 segue que

$$
\left(\gamma_{1}, \gamma_{2}\right)\left(\left(\tau_{1}, \tau_{2}\right) v\right)=\gamma_{1}\left(\gamma_{2}\left(\tau_{1}\left(\tau_{2} v\right)\right)\right)
$$

Por outro lado, temos

$$
\left.\left(\left(\gamma_{1}, \gamma_{2}\right) \cdot \mu\left(\tau_{1}, \tau_{2}\right)\right) v=\gamma_{1} \mu\left(\gamma_{2}\right) \tau_{1}\left(\gamma_{2} \tau_{2} v\right)\right)
$$

Assim, em termos de representação, 2.3 ocorre se, e somente se,

$$
\rho\left(\gamma_{1}\right) \eta\left(\gamma_{2}\right) \rho\left(\tau_{1}\right) \eta\left(\tau_{2}\right)=\rho\left(\gamma_{1}\right) \rho\left(\mu\left(\gamma_{2}\right) \tau_{1}\right) \eta\left(\gamma_{2}\right) \eta\left(\tau_{2}\right)
$$


ou seja, se, e somente se,

$$
\eta\left(\gamma_{2}\right) \rho\left(\tau_{1}\right)=\rho\left(\mu\left(\gamma_{2}\right) \tau_{1}\right) \eta\left(\gamma_{2}\right)
$$

Observamos que a igualdade

$$
\rho\left(\gamma_{1}\right) \eta\left(\gamma_{2}\right)=\eta\left(\gamma_{2}\right) \rho\left(\mu\left(\gamma_{2}^{-1}\right)\left(\gamma_{1}\right)\right)
$$

destaca a não comutatividade das ações de $\Gamma_{1}$ e $\Gamma_{2}$ se $\mu$ é não trivial.

Note ainda que quando $\mu$ é o homomorfismo trivial, levando todo elemento de $\Gamma_{2}$ no automorfismo identidade de $\Gamma_{1}$, então $\Gamma_{1} \rtimes \Gamma_{2}$ é o produto direto $\Gamma_{1} \times \Gamma_{2}$. Neste caso, a operação em 2.2 é uma ação se, e somente se, as ações de $\Gamma_{1}$ e $\Gamma_{2}$ comutam. Ainda, se $\Gamma_{1}$ e $\Gamma_{2}$ possuem simetrias e antissimentrias simultaneamente, então para cada $\gamma_{2} \in \Gamma_{2} \mathrm{o}$ automorfismo $\mu\left(\gamma_{2}\right): \Gamma_{1} \rightarrow \Gamma_{1}$ da Proposição 2.1.1 preserva as simetrias de $\Gamma_{1}$, ou seja, $\mu\left(\gamma_{2}\right)\left(\gamma_{1}\right)$ é uma simetria de $\Gamma_{1}$ se, e somente se, $\gamma_{1}$ é simetria em $\Gamma_{1}$.

Neste trabalho assumimos que $\Gamma_{1}$ e $\Gamma_{2}$ admitem um produto semi-direto com uma representação nas condições da Proposição 2.1.1. Note que esta hipótese pode falhar, por exemplo, considere os grupos $\Gamma_{1}=\left\langle\kappa_{1}\right\rangle$ e $\Gamma_{2}=\left\langle\kappa_{2}\right\rangle$ agindo em $\mathbb{R}^{2}$ como $\kappa_{1}(x, y)=(y, x)$ e $\kappa_{2}(x, y)=(x,-y)$. Neste caso, não existe uma representação do produto direto $\Gamma_{1} \times \Gamma_{2} \mathrm{em}$ $\mathbb{R}^{2}$ como dado na Proposição 2.1.1. Porém, existem aplicações em que se deseja trabalhar com o grupo gerado pelas duas involuções anteriores, $\Gamma=\left\langle\kappa_{1}, \kappa_{2}\right\rangle$. Neste caso, o que temos é $\Gamma=\tilde{\Gamma}_{1} \rtimes \tilde{\Gamma}_{2} \operatorname{com} \tilde{\Gamma}_{1}=\left\langle\kappa_{1} \circ \kappa_{2}\right\rangle$ e $\tilde{\Gamma}_{2}=\left\langle\kappa_{2}\right\rangle$.

Ao longo deste trabalho, denotamos por $(\rho \eta, V)$ a representação do produto semi-direto $\Gamma_{1} \rtimes \Gamma_{2}$, proveniente da ação produto definida em (2.2).

Agora, sejam $\Gamma_{1}$ e $\Gamma_{2}$ grupos, não necessariamente compactos, e $\sigma_{1}$ e $\sigma_{2}$ homomorfismos definidos como em (1.4). Introduzimos o homomorfismo $\tilde{\sigma}$ como

$$
\begin{aligned}
\tilde{\sigma}: \Gamma_{1} \rtimes \Gamma_{2} & \rightarrow \mathbf{Z}_{2} \\
\left(\gamma_{1}, \gamma_{2}\right) & \mapsto \sigma_{1}\left(\gamma_{1}\right) \sigma_{2}\left(\gamma_{2}\right)
\end{aligned}
$$

A estrutura de $\tilde{\sigma}$ fornece uma relação entre a teoria invariante para o produto $\Gamma_{1} \rtimes \Gamma_{2}$ e a teoria invariante para cada grupo, $\Gamma_{1}$ e $\Gamma_{2}$, separadamente:

Proposição 2.1.2. Nas condições acima valem as seguintes igualdades:

1) $\mathcal{P}_{V}\left(\Gamma_{1} \rtimes \Gamma_{2}\right)=\mathcal{P}_{V}\left(\Gamma_{1}\right) \cap \mathcal{P}_{V}\left(\Gamma_{2}\right)$; 
2) $\overrightarrow{\mathcal{P}}_{V}\left(\Gamma_{1} \rtimes \Gamma_{2}\right)=\overrightarrow{\mathcal{P}}_{V}\left(\Gamma_{1}\right) \cap \overrightarrow{\mathcal{P}}_{V}\left(\Gamma_{2}\right)$;

3) $\mathcal{Q}_{V}\left(\Gamma_{1} \rtimes \Gamma_{2}\right)=\mathcal{Q}_{V}\left(\Gamma_{1}\right) \cap \mathcal{Q}_{V}\left(\Gamma_{2}\right)$;

4) $\overrightarrow{\mathcal{Q}}_{V}\left(\Gamma_{1} \rtimes \Gamma_{2}\right)=\overrightarrow{\mathcal{Q}}_{V}\left(\Gamma_{1}\right) \cap \overrightarrow{\mathcal{Q}}_{V}\left(\Gamma_{2}\right)$.

Demonstração: Se $f \in \mathcal{P}_{V}\left(\Gamma_{1} \rtimes \Gamma_{2}\right)$, então $f\left(\gamma_{1}\left(\gamma_{2} v\right)\right)=f(v)$. Para $\gamma_{1}=I d$ temos $f\left(\gamma_{2} v\right)=f(v)$, ou seja, $f \in \mathcal{P}_{V}\left(\Gamma_{2}\right)$ e, se $\gamma_{2}=I d$, então $f\left(\gamma_{1} v\right)=f(v)$ implicando em $f \in \mathcal{P}_{V}\left(\Gamma_{1}\right)$. Por outro lado, se $f \in \mathcal{P}_{V}\left(\Gamma_{1}\right) \cap \mathcal{P}_{V}\left(\Gamma_{2}\right)$ segue que $f\left(\gamma_{1}\left(\gamma_{2} v\right)\right)=f\left(\gamma_{2} v\right)=$ $f(v)$.

Os outros itens seguem de forma análoga ao primeiro.

Se $\Gamma_{1}$ possui apenas simetrias, então os itens 3 e 4 da Proposição 2.1 .2 ficam:

$$
\mathcal{Q}_{V}\left(\Gamma_{1} \rtimes \Gamma_{2}\right)=\mathcal{P}_{V}\left(\Gamma_{1}\right) \cap \mathcal{Q}_{V}\left(\Gamma_{2}\right) \quad \text { e } \quad \overrightarrow{\mathcal{Q}}_{V}\left(\Gamma_{1} \rtimes \Gamma_{2}\right)=\overrightarrow{\mathcal{P}}_{V}\left(\Gamma_{1}\right) \cap \overrightarrow{\mathcal{Q}}_{V}\left(\Gamma_{2}\right) .
$$

A Proposição 2.1.2 é usada mais à frente para provar os Lemas 2.2.1 e 2.2.2. Estes lemas, por sua vez, são usados para a dedução de formas normais de sistemas $\Gamma$-reversíveisequivariantes, onde a abordagem algébrica é aplicada a $\mathbf{S} \rtimes \Gamma$ para um grupo conveniente $\mathbf{S}$ relacionado a este sistema. Isto é feito em detalhes no Capítulo 5.

\subsection{Representação diagonal no contexto invariante}

Nesta seção, sejam $\Gamma_{1}$ e $\Gamma_{2}$ grupos quaisquer e $(\rho, V)$ e $(\eta, W)$ representações de $\Gamma_{1}$ e $\Gamma_{2}$, respectivamente, sendo $V$ e $W$ espaços reais de dimensão finita. As ações naturais de $\Gamma_{1}$ e $\Gamma_{2}$ induzidas em $V \times W$ são da forma

$$
\gamma_{1}(v, w)=\left(\gamma_{1} v, w\right) \quad \text { e } \quad \gamma_{2}(v, w)=\left(v, \gamma_{2} w\right)
$$

respectivamente. Temos também a ação natural do grupo $\Gamma_{1} \times \Gamma_{2}$ em $V \times W$ dada pela ação diagonal:

$$
\left(\gamma_{1}, \gamma_{2}\right)(v, w)=\left(\gamma_{1} v, \gamma_{2} w\right)
$$

Neste caso, a representação diagonal de $\left(\gamma_{1}, \gamma_{2}\right) \in \Gamma_{1} \times \Gamma_{2}$ é denotada por $\left(\rho\left(\gamma_{1}\right), \eta\left(\gamma_{2}\right)\right)$ e corresponde à matriz

$$
\left(\begin{array}{cc}
\rho\left(\gamma_{1}\right) & 0 \\
0 & \eta\left(\gamma_{2}\right)
\end{array}\right)
$$


em $\mathbf{G L}(V \times W)$. Com esta formalização apresentamos dois lemas que são usados na obtenção das formas normais do Capítulo 5 .

Lema 2.2.1. Sejam $\Gamma_{1}$ e $\Gamma_{2}$ grupos agindo em $V$ e $W$, respectivamente. Sejam $u_{1}, \ldots, u_{r} e$ $\alpha_{1}, \ldots, \alpha_{s}$ bases de Hilbert para $\mathcal{P}_{V}\left(\Gamma_{1}\right)$ e $\mathcal{P}_{W}\left(\Gamma_{2}\right)$, respectivamente. Então $u_{1}, \ldots u_{r}, \alpha_{1}, \ldots, \alpha_{s}$ é uma base de Hilbert para o anel $\mathcal{P}_{V \times W}\left(\Gamma_{1} \rtimes \Gamma_{2}\right)$.

Lema 2.2.2. Sejam $\Gamma_{1}$ e $\Gamma_{2}$ grupos agindo em $V$ e $W$, respectivamente. Sejam $u_{1}, \ldots, u_{r}$ e $\alpha_{1}, \ldots, \alpha_{s}$ bases de Hilbert para $\mathcal{P}_{V}\left(\Gamma_{1}\right)$ e $\mathcal{P}_{W}\left(\Gamma_{2}\right)$, respectivamente. Sejam $f_{1}, \ldots f_{m}$ geradores para $\overrightarrow{\mathcal{P}}_{V}\left(\Gamma_{1}\right)$ sobre o anel $\mathcal{P}_{V}\left(\Gamma_{1}\right)$, e sejam $g_{1}, \ldots g_{n}$ geradores para $\overrightarrow{\mathcal{P}}_{W}\left(\Gamma_{2}\right)$ sobre o anel $\mathcal{P}_{W}\left(\Gamma_{2}\right)$. Então, o módulo $\overrightarrow{\mathcal{P}}_{V \times W}\left(\Gamma_{1} \rtimes \Gamma_{2}\right)$ é gerado sobre o anel $\mathcal{P}_{V \times W}\left(\Gamma_{1} \rtimes \Gamma_{2}\right)$ pelas aplicações

$$
\left(\begin{array}{c}
f_{i} \\
0_{W}
\end{array}\right) \quad e\left(\begin{array}{c}
0_{V} \\
g_{j}
\end{array}\right), \quad \forall 1 \leq i \leq m \quad \text { e } 1 \leq j \leq n
$$

Demonstração: Seja $H \in \overrightarrow{\mathcal{P}}_{V \times W}\left(\Gamma_{1} \rtimes \Gamma_{2}\right)$. Podemos escrever $H$ como $H=(p, q)$ com $p: V \times W \rightarrow V$ e $q: V \times W \rightarrow W$. Pela equivariância de $H$ temos:

$$
p\left(\gamma_{1} v, \gamma_{2} w\right)=\gamma_{1} p(v, w) \quad \text { e } \quad q\left(\gamma_{1} v, \gamma_{2} w\right)=\gamma_{2} q(v, w)
$$

A primeira condição implica que $p$ é $\Gamma_{1}$-equivariante em $v$ e cada coordenada de $p$ é $\Gamma_{2^{-}}$ invariante em $w$. Para cada $w$ fixado definimos $f_{w}(v)=p(v, w)$ e obtemos $f_{w} \in \overrightarrow{\mathcal{P}}_{V}\left(\Gamma_{1}\right)$. Portanto, $f_{w}(v)=\sum_{i=1}^{m} h_{i}\left(u_{1}(v), \ldots, u_{r}(v), w\right) f_{i}(v)$ sendo cada $h_{i} \Gamma_{1}$-invariante. Mas, cada coordenada de $p$ é $\Gamma_{2}$-invariante em $w$, logo $h_{i}$ depende de funções $\Gamma_{2}$-invariantes em $w$, ou seja,

$$
p(v, w)=\sum_{i=1}^{m} h_{i}\left(u_{1}(v), \ldots, u_{r}(v), \alpha_{1}(w), \ldots, \alpha_{s}(w)\right) f_{i}(v),
$$

$\operatorname{com} h_{i} \in \mathcal{P}_{V \times W}\left(\Gamma_{1} \rtimes \Gamma_{2}\right)$.

De forma análoga, consideramos a segunda condição e provamos que

$$
q(v, w)=\sum_{j=1}^{n} \tilde{h}_{j}\left(u_{1}, \ldots, u_{r}, \alpha_{1}, \ldots, \alpha_{s}\right) g_{j}(w),
$$

$\operatorname{com} \tilde{h}_{j} \in \mathcal{P}_{V \times W}\left(\Gamma_{1} \rtimes \Gamma_{2}\right)$.

Registramos a seguir um exemplo que será usado no Capítulo 5 na construção de formas normais de campos de vetores $\mathbf{S} \times \mathbf{T}^{n}$-reversíveis-equivariantes em $\mathbb{C}^{n+1}$ para casos ressonantes e não ressonantes. Quando $n=1$ então $\mathbf{T}=\mathbf{S}^{1}$. 
Exemplo 2.2.3. Considere a ação do grupo $\mathbf{S}^{1}$ em $\mathbb{C}$ dada por $\theta z=e^{i \theta} z$. É fácil ver que $\mathcal{P}_{\mathbb{C}}\left(\mathbf{S}^{1}\right)=\langle z \bar{z}\rangle$ e $\overrightarrow{\mathcal{P}}_{\mathbb{C}}\left(\mathbf{S}^{1}\right)=\mathcal{P}_{\mathbb{C}}\left(\mathbf{S}^{1}\right)\{z, i z\}$ (veja [35, Capítulo VII, Seções 4 e 5]). A ação diagonal de $\left(\mathbf{S}^{1}\right)^{n}$ em $\mathbb{C}^{n}$ é dada por $\left(\theta_{1}, \ldots, \theta_{n}\right)\left(z_{1}, \ldots z_{n}\right)=\left(e^{i \theta_{1}} z_{1}, \ldots, e^{i \theta_{n}} z_{n}\right)$.

Do Lema 2.2.1. $\left|z_{1}\right|^{2}, \ldots,\left|z_{n}\right|^{2}$ é uma base de Hilbert para $\mathcal{P}_{\mathbb{C}^{n}}\left(\left(\mathbf{S}^{1}\right)^{n}\right)$. Do Lema 2.2.2 obtemos um conjunto de geradores para $\mathcal{P}_{\mathbb{C}^{n}}\left(\left(\mathbf{S}^{1}\right)^{n}\right)$ sobre $\mathcal{P}_{\mathbb{C}^{n}}\left(\left(\mathbf{S}^{1}\right)^{n}\right)$ :

$$
\begin{gathered}
\left(z_{1}, 0,0, \ldots, 0\right),\left(i z_{1}, 0,0, \ldots, 0\right),\left(0, z_{2}, 0, \ldots, 0\right),\left(0, i z_{2}, 0, \ldots, 0\right), \ldots \\
\left(0, \ldots, 0,0, z_{n}\right) \text { e }\left(0, \ldots, 0,0, i z_{n}\right)
\end{gathered}
$$

\subsection{Séries de Hilbert e fórmulas de Molien para a ação diagonal}

Sejam $(\rho, V)$ e $(\eta, W)$ representações de grupos de Lie compactos $\Gamma_{1}$ e $\Gamma_{2}$ em $V$ e $W$, respectivamente, e considere a representação diagonal de $\Gamma_{1} \times \Gamma_{2}$ em $V \times W$ definida em (2.6). Como falamos na Seção 2.2 denotamos por $\left(\rho\left(\gamma_{1}\right), \eta\left(\gamma_{2}\right)\right)$ a representação diagonal $\operatorname{de}\left(\gamma_{1}, \gamma_{2}\right) \in \Gamma_{1} \times \Gamma_{2}$.

Semelhante ao apresentado na Seção 1.5, existem fórmulas explícitas para as séries de Hilbert para os invariantes e equivariantes segundo a ação de $\Gamma_{1} \times \Gamma_{2}$. Registramos nesta seção que estas fórmulas podem ser obtidas a partir das fórmulas para as séries de Hilbert dos invariantes e equivariantes de cada grupo, separadamente.

Os resultados desta seção são ferramentas úteis no estudo por exemplo de sistemas de células acopladas, descritos mais a frente, e também no cálculo de formas normais de sistemas em que o grupo $\mathbf{S}$ definido em (3.8) é compacto e possui uma estrutura de produto cartesiano agindo de forma diagonal num espaço vetorial. No caso da obtenção de formas normais, como já falamos desde o início, a forma obtida é uma série formal e, neste caso, é preciso tomar grau a grau os termos que aparecem na forma normal. Sendo assim, as fórmulas de Molien são úteis para sabermos a quantidade de elementos em cada grau que devemos considerar.

Se $\chi_{V}$ e $\chi_{X}$ são os caracteres das representações $(\rho, V)$ e $(\eta, W)$, respectivamente, então o caracter da representação de $\Gamma_{1} \times \Gamma_{1}$ é dado por

$$
\chi_{V \times W}\left(\gamma_{1}, \gamma_{2}\right)=\chi_{V}\left(\gamma_{1}\right)+\chi_{W}\left(\gamma_{2}\right) .
$$


Proposição 2.3.1. Sejam $\Gamma_{1}$ e $\Gamma_{2}$ grupos de Lie compactos agindo em $V$ e $W$, respectivamente, e considere a ação diagonal de $\Gamma_{1} \times \Gamma_{1}$ em $V \times W$. Sejam $\Phi_{V}^{\Gamma_{1}}$ e $\Phi_{W}^{\Gamma_{2}}$ as séries de Hilbert para $\mathcal{P}_{V}\left(\Gamma_{1}\right)$ e $\mathcal{P}_{W}\left(\Gamma_{2}\right)$, respectivamente, e sejam $\Psi_{V}^{\Gamma_{1}}$ e $\Psi_{W}^{\Gamma_{2}}$ as séries de Hilbert para $\overrightarrow{\mathcal{P}}_{V}\left(\Gamma_{1}\right)$ e $\overrightarrow{\mathcal{P}}_{W}\left(\Gamma_{2}\right)$, respectivamente. Então, as séries de Hilbert para $\mathcal{P}_{V \times W}\left(\Gamma_{1} \times \Gamma_{2}\right)$ e $\overrightarrow{\mathcal{P}}_{V \times W}\left(\Gamma_{1} \times \Gamma_{2}\right)$ são dadas, respectivamente, por

$$
\Phi_{V \times W}^{\Gamma_{1} \times \Gamma_{2}}(t)=\Phi_{\Gamma_{1}}(t) \Phi_{\Gamma_{2}}(t)
$$

$e$

$$
\Psi_{V \times W}^{\Gamma_{1} \times \Gamma_{2}}(t)=\Psi_{V}^{\Gamma_{1}}(t) \Phi_{W}^{\Gamma_{2}}(t)+\Psi_{W}^{\Gamma_{2}}(t) \Phi_{V}^{\Gamma_{1}}(t)
$$

Demonstração: A dedução é feita usando as fórmulas de Molien (Teoremas 1.5.1 e 1.5.2) e a linearidade da integral de Haar:

$$
\begin{aligned}
\Phi_{V \times W}^{\Gamma_{1} \times \Gamma_{2}}(t) & =\int_{\Gamma_{2}}\left(\int_{\Gamma_{1}} \frac{1}{\operatorname{det}\left(1-t\left(\rho\left(\gamma_{1}\right), \eta\left(\gamma_{2}\right)\right)\right)} d \gamma_{1}\right) d \gamma_{2} \\
& =\int_{\Gamma_{2}}\left(\int_{\Gamma_{1}} \frac{1}{\operatorname{det}\left(1-t \rho\left(\gamma_{1}\right)\right) \operatorname{det}\left(1-t \eta\left(\gamma_{2}\right)\right)} d \gamma_{1}\right) d \gamma_{2} \\
& =\int_{\Gamma_{2}}\left(\frac{1}{\operatorname{det}\left(1-t \eta\left(\gamma_{2}\right)\right)} \int_{\Gamma_{1}} \frac{1}{\operatorname{det}\left(1-t \rho\left(\gamma_{1}\right)\right)} d \gamma_{1}\right) d \gamma_{2} \\
& =\int_{\Gamma_{2}}\left(\frac{1}{\operatorname{det}\left(1-t \eta\left(\gamma_{2}\right)\right)} \Phi_{V}^{\Gamma_{1}}(t)\right) d \gamma_{2} \\
& =\Phi_{V}^{\Gamma_{1}}(t) \int_{\Gamma_{2}}\left(\frac{1}{\operatorname{det}\left(1-t \eta\left(\gamma_{2}\right)\right)}\right) d \gamma_{2}=\Phi_{V}^{\Gamma_{1}}(t) \Phi_{W}^{\Gamma_{2}}(t)
\end{aligned}
$$

Para os equivariantes temos

$$
\begin{aligned}
\Psi_{V \times W}^{\Gamma_{1} \times \Gamma_{2}}(t) & =\int_{\Gamma_{2}}\left(\int_{\Gamma_{1}} \frac{\chi_{V \times W}\left(\left(\gamma_{1}, \gamma_{2}\right)^{-1}\right)}{\operatorname{det}\left(1-t\left(\rho\left(\gamma_{1}\right), \eta\left(\gamma_{2}\right)\right)\right)}\right) d \gamma_{2} \\
& =\int_{\Gamma_{2}}\left(\int_{\Gamma_{1}} \frac{\chi_{V}\left(\gamma_{1}^{-1}\right)+\chi_{W}\left(\gamma_{2}^{-1}\right)}{\operatorname{det}\left(1-t \rho\left(\gamma_{1}\right)\right) \operatorname{det}\left(1-t \eta\left(\gamma_{2}\right)\right)} d \gamma_{1}\right) d \gamma_{2} \\
& =\int_{\Gamma_{2}} \frac{1}{\operatorname{det}\left(1-t \rho\left(\gamma_{2}\right)\right)}\left(\int_{\Gamma_{1}} \frac{\chi_{V}\left(\gamma_{1}^{-1}\right)}{\operatorname{det}\left(1-t \rho\left(\gamma_{1}\right)\right)} d \gamma_{1}+\int_{\Gamma_{1}} \frac{\chi_{W}\left(\gamma_{2}^{-1}\right)}{\operatorname{det}\left(1-t \rho\left(\gamma_{1}\right)\right)} d \gamma_{1}\right) d \gamma_{2} \\
& =\int_{\Gamma_{2}} \frac{1}{\operatorname{det}\left(1-t \rho\left(\gamma_{2}\right)\right)}\left(\Psi_{\Gamma_{1}}(t)+\chi_{W}\left(\gamma_{2}^{-1}\right) \Phi_{V}^{\Gamma_{1}}(t)\right) d \gamma_{2} \\
& =\Psi_{V}^{\Gamma_{1}}(t) \int_{\Gamma_{2}} \frac{1}{\operatorname{det}\left(1-t \rho\left(\gamma_{2}\right)\right)} d \gamma_{2}+\Phi_{V}^{\Gamma_{1}}(t) \int_{\Gamma_{2}} \frac{\chi_{W}\left(\gamma_{2}^{-1}\right)}{\operatorname{det}\left(1-t \rho\left(\gamma_{2}\right)\right)} \\
& =\Psi_{V}^{\Gamma_{1}}(t) \Phi_{W}^{\Gamma_{2}}(t)+\Phi_{V}^{\Gamma_{1}}(t) \Psi_{W}^{\Gamma_{2}}(t) .
\end{aligned}
$$


Para ilustrar os resultados apresentados nesta seção, apresentamos um exemplo onde obtemos o número de geradores para o espaço vetorial dos invariantes e equivariantes de cada grau segundo a ação de um produto diagonal.

Exemplo 2.3.2. Considere as ações de $\mathbf{S}^{1}$ e $\mathbf{Z}_{2}$ em $\mathbb{C}$ e $\mathbb{R}^{2}$, respectivamente, dadas por

$$
\theta z=e^{i \theta} z \quad \text { e } \quad \kappa\left(x_{1}, x_{2}\right)=\left(x_{1},-x_{2}\right)
$$

onde $\theta \in \mathbf{S}^{1}$ e $\kappa$ é o gerador de $\mathbf{Z}_{2}$.

Consideramos as fórmulas de Molien para $\mathcal{P}_{\mathbb{C}}\left(\mathbf{S}^{1}\right)$ e $\overrightarrow{\mathcal{P}}_{\mathbb{C}}\left(\mathbf{S}^{1}\right)$ calculadas em [5]:

$$
\Phi_{\mathbb{C}}^{\mathbf{S}^{1}}(t)=\frac{1}{1-t^{2}} \quad \text { e } \quad \Psi_{\mathbb{C}}^{\mathbf{S}^{1}}=\frac{2 t}{1-t^{2}}
$$

Por cálculo direto das definições obtemos as fórmulas de Molien para $\mathcal{P}_{\mathbb{R}^{2}}\left(\mathbf{Z}_{2}\right)$ e $\overrightarrow{\mathcal{P}}_{\mathbb{R}^{2}}\left(\mathbf{Z}_{2}\right)$,

$$
\Phi_{\mathbb{R}^{2}}^{\mathbf{Z}_{2}}(t)=\frac{1}{2}\left(\frac{1}{(1-t)^{2}}+\frac{1}{1-t^{2}}\right) \quad \text { e } \quad \Psi_{\mathbb{R}^{2}}^{\mathbf{Z}_{2}}(t)=\frac{1}{(1-t)^{2}}
$$

Pela Proposição 2.3.1 temos

$$
\begin{aligned}
& \Phi_{\mathbb{C} \times \mathbb{R}^{2}}^{\mathbf{S}^{1}(t)}=\frac{1}{2}\left(\frac{1}{(1-t)^{2}}+\frac{1}{1-t^{2}}\right)\left(\frac{1}{1-t^{2}}\right), \\
& \Psi_{\mathbb{C} \times \mathbb{R}^{2}}^{\mathbf{S}^{1} \times \mathbf{Z}_{2}}(t)=\left(\frac{1}{(1-t)^{2}}\right)\left(\frac{1}{1-t^{2}}\right)+\frac{1}{2}\left(\frac{2 t}{1-t^{2}}\right)\left(\frac{1}{(1-t)^{2}}+\frac{1}{1-t^{2}}\right) .
\end{aligned}
$$

Desenvolvendo estas séries obtemos

$$
\begin{gathered}
\Phi_{\mathbb{C} \times \mathbb{R}^{2}}^{\mathbf{S}^{1} \times \mathbf{Z}_{2}}(t)=1+t+3 t^{2}+3 t^{3}+6 t^{4}+6 t^{5}+10^{6}+\ldots \\
\Psi_{\mathbb{C} \times \mathbb{R}^{2}}^{\mathbf{S}^{1} \times \mathbf{Z}_{2}}(t)=1+4 t+6 t^{2}+12 t^{3}+15 t^{4}+25 t^{5}+\ldots
\end{gathered}
$$

Assim, neste exemplo, para os invariantes temos: 1 gerador de grau 1, 3 geradores de grau 2, 3 geradores de grau 3, 6 geradores de grau 4, e assim por diante. Analogamente, para os equivariantes temos: 4 geradores de grau 1, 6 geradores de grau 2, 12 geradores de grau 3, e assim por diante.

Paralelamente, é conhecido que

$$
\mathcal{P}_{\mathbb{C}}\left(\mathbf{S}^{1}\right)=\langle z \bar{z}\rangle \quad \text { e } \quad \overrightarrow{\mathcal{P}}_{\mathbb{C}}\left(\mathbf{S}^{1}\right)=\mathcal{P}_{\mathbb{C}}\left(\mathbf{S}^{1}\right)\{z, i z\}
$$




$$
\mathcal{P}_{\mathbb{R}^{2}}\left(\mathbf{Z}_{2}\right)=\left\langle x_{1}, x_{2}^{2}\right\rangle \quad \text { e } \quad \overrightarrow{\mathcal{P}}_{\mathbb{R}^{2}}\left(\mathbf{Z}_{2}\right)=\mathcal{P}_{\mathbb{R}^{2}}\left(\mathbf{Z}_{2}\right)\left\{(1,0),\left(0, x_{2}\right)\right\}
$$

Dos Lemas 2.2.1 e 2.2.2 segue que

$$
\begin{gathered}
\mathcal{P}_{\mathbb{C} \times \mathbb{R}^{2}}\left(\mathbf{S}^{1} \times \mathbf{Z}_{2}\right)=\left\langle z \bar{z}, x_{1}, x_{2}^{2}\right\rangle, \\
\overrightarrow{\mathcal{P}}_{\mathbb{C} \times \mathbb{R}^{2}}\left(\mathbf{S}^{1} \times \mathbf{Z}_{2}\right)=\mathcal{P}_{\mathbb{C} \times \mathbb{R}^{2}}\left(\mathbf{S}^{1} \times \mathbf{Z}_{2}\right)\left\{(z, 0,0),(i z, 0,0),(0,1,0),\left(0,0, x_{2}\right)\right\} .
\end{gathered}
$$

Desta forma, fica evidenciado que os coeficientes das séries em 2.12 são as dimensões dos espaços vetoriais dos invariantes e equivariantes de cada grau segundo a ação de $\mathbf{S}^{1} \times \mathbf{Z}_{2}$.

Para a representação diagonal do produto $\Gamma^{N}$ em $V^{N}$, usamos indução sobre $N$ em 2.10) e 2.11 e obtemos:

Corolário 2.3.3. Seja $\Gamma$ um grupo de Lie compacto agindo em $V$, e sejam $\Phi_{\Gamma}$ e $\Psi_{\Gamma}$ as séries de Hilbert para $\mathcal{P}_{V}(\Gamma)$ e $\overrightarrow{\mathcal{P}}_{V}(\Gamma)$, respectivamente. Então,

$$
\Phi_{V^{N}}^{\Gamma^{N}}(t)=\left(\Phi_{V}^{\Gamma}(t)\right)^{N} \quad \text { e } \quad \Psi_{V^{N}}^{\Gamma^{N}}(t)=N \Psi_{V}^{\Gamma}(t)\left(\Phi_{V}^{\Gamma}(t)\right)^{N-1}
$$

Do ponto de vista de aplicações, os Lemas 2.2.1 e 2.2.2, assim como a Proposição 2.3.1. também podem ser úteis para o estudo de sistemas de células acopladas, não necessariamente idênticas, que possuem simetrias internas. A formalização da teoria de sistemas de células acopladas que possuem apenas simetrias pode ser encontrada em [25, 26], e com base nestes trabalhos apresentamos um breve resumo.

Em 25] é observado que a forma natural para um sistema com $N$ células idênticas acopladas com mesmo tipo de acoplamento entre todas as células é

$$
\dot{x}_{j}=f\left(x_{j}\right)+\sum_{i=1}^{N} C(i, j) h\left(x_{i}, x_{j}\right),
$$

com $x_{j} \in V$, para $1 \leq i, j \leq N, f: V \rightarrow V$ representando a dinâmica interna de cada célula, $h: V \times V \rightarrow V$ sendo o tipo de acoplamento entre a célula $i$ e a célula $j$ e $C$ uma matriz de conexão definida como

$$
C(i, j)=\left\{\begin{array}{l}
1, \text { se a célula } i \text { está acoplada à célula } j ; \\
0, \text { caso contrário. }
\end{array}\right.
$$

Nos referimos ao sistema 2.14 como $\dot{x}=F(x)$ para $x=\left(x_{1}, \ldots, x_{N}\right)$ e $F=$ $\left(F_{1}, \ldots, F_{N}\right) \operatorname{com} F_{j}(x)=f\left(x_{j}\right)+\sum_{i=1} C(i, j) h\left(x_{i}, x_{j}\right), \quad 1 \leq i, j \leq N$. 
Agora, discutimos as simetrias de $F$. Existem dois tipos de simetrias para $F$ : simetrias internas e simetrias globais. Com a hipótese de todas as células estarem acopladas 1 a 1 , o grupo de simetrias globais aqui é o grupo de permutações de $N$ elementos $\mathbf{S}_{N}$, de forma que

$$
\tau C \tau^{-1}=C
$$

A ação de $\mathbf{S}_{N}$ em $V^{N}$ é definida como

$$
\tau\left(x_{1}, \ldots x_{N}\right)=\left(x_{\tau^{-1}(1)}, \ldots, x_{\tau^{-1}(N)}\right)
$$

Neste caso, temos

$$
F(\tau x)=\tau F(x), \quad \forall x \in V^{N}, \quad \forall \tau \in \mathbf{S}_{N}
$$

Esta condição de equivariância nos diz que as simetrias de $\mathbf{S}_{N}$ permutam as células sem mudar o sistema de equações.

Dizemos que $\Gamma$ é um grupo de simetrias internas (de cada célula de (2.14)) se $f \in \overrightarrow{\mathcal{P}}_{V}(\Gamma)$ e

$$
h\left(\gamma_{i} x_{i}, x_{j}\right)=h\left(x_{i}, x_{j}\right) \quad \text { e } \quad h\left(x_{i}, \gamma_{j} x_{j}\right)=\gamma_{j} h\left(x_{i}, x_{j}\right)
$$

para cada $\gamma_{i}, \gamma_{j} \in \Gamma, 1 \leq i, j \leq N$.

Desta forma, $F$ é em particular $\Gamma^{N}$-equivariante segundo a ação diagonal de $\Gamma^{N}$ em $V^{N}$ definida em (2.6).

O grupo de simetrias do sistema (2.14) é o produto coroa $\Gamma$ 々 $\mathbf{S}_{N}$, ou seja, o produto semi-direto de $\Gamma^{N}$ por $\mathbf{S}_{N}$. Neste caso, é preciso reconhecer a forma geral das aplicações $\Gamma$ ? $\mathbf{S}_{N}$-equivariantes.

Portanto, da Proposição 2.1.2 o ponto de partida para o estudo de sistemas de células acopladas é determinar a forma geral das aplicações em $\mathcal{P}_{V^{N}}\left(\Gamma^{N}\right)$, e depois impor as simetrias de $\mathbf{S}_{N}$. Para isto, reconhecemos o grupo de simetrias $\Gamma$ de cada célula, determinamos geradores para o módulo $\overrightarrow{\mathcal{P}}_{V}(\Gamma)$ sobre o anel $\mathcal{P}_{V}(\Gamma)$, e usamos os Lemas 2.2.1 e 2.2 .2 para construir os geradores para o módulo $\overrightarrow{\mathcal{P}}_{V^{N}}\left(\Gamma^{N}\right)$ sobre o anel $\mathcal{P}_{V^{N}}\left(\Gamma^{N}\right)$. Em seguida, impomos as simetrias de $\mathbf{S}_{N}$.

É claro que, a forma para se obter estes geradores não é única, porém usando as ferramentas deste capítulo este processo se torna mais direto. Veja por exemplo [24] onde a autora obtém a forma geral de um sistema com $N$ células idênticas acopladas com cada célula $\mathbf{O}(2)$-equivariante, através da definição direta da ação de $\mathbf{O}(2)^{N}$ em $V^{N}$. 
O que observamos é que semelhante ao caso equivariante, também é possível acoplar células que possuem simetrias e antissimetrias. A formalização para este caso é supor que cada célula é $\Gamma$-reversível-equivariante, ou seja, $f \in \overrightarrow{\mathcal{Q}}_{V}(\Gamma)$ e

$$
h\left(\gamma x_{i}, x_{j}\right)=h\left(x_{i}, x_{j}\right) \quad \text { e } \quad h\left(x_{i}, \gamma x_{j}\right)=\sigma\left(\gamma_{i}\right) \gamma_{i} h\left(x_{i}, x_{j}\right)
$$

para cada $\gamma_{i}, \gamma_{j} \in \Gamma, 1 \leq i, j \leq N$, onde $\sigma$ é definido em (1.4).

Para este caso, temos $F\left(\gamma_{1} x_{1}, \ldots, \gamma_{N} x_{N}\right)=\left(\sigma\left(\gamma_{1}\right) \gamma_{1} F_{1}(x), \ldots, \sigma\left(\gamma_{N}\right) \gamma_{N} F_{N}(x)\right)$ para cada $\gamma_{i} \in \Gamma$, ou seja, cada célula é $\Gamma$-equivariante se considerarmos a ação dual de $\Gamma$ na meta. Segue dos Lemas 2.2.1 e 2.2.2 que para conhecermos a forma geral de $F$ basta conhecermos os geradores de $\overrightarrow{\mathcal{Q}}_{V}(\Gamma)$ e $\mathcal{P}_{V}(\Gamma)$. 



$\frac{-10}{3}$

\section{Forma normal reversível equivariante}

A teoria de forma normal tem sido uma importante ferramenta para o estudo local em sistemas dinâmicos sob diferentes aspectos. Temos evidenciado um interesse grande neste método para o estudo, por exemplo, de bifurcações e ocorrência de ciclos limites no contexto reversível equivariante. O fato é o interesse em se poder assumir que as equações estejam numa forma mais simples, obtida através de mudanças de coordenadas em cada grau da expansão da série de Taylor, mudanças estas que preservem propriedades a serem investigadas. Por exemplo, quando o sistema é $\Gamma$-reversível-equivariante então as mudanças de coordenadas devem ser $\Gamma$-equivariantes.

A forma normal introduz, naturalmente, um novo grupo de simetrias que juntamente com o grupo $\Gamma$ forma um produto semi-direto. Usando ferramentas desenvolvidas no Capítulo 2 desenvolvemos um método algébrico para obter a forma normal $\Gamma$-reversívelequivariante de campos de vetores num espaço vetorial real de dimensão finita $V$. Como veremos, este método leva em consideração a ação de $\Gamma$ para simplificar a dedução da forma normal. 
Na Seção 3.1 apresentamos a teoria de forma normal desenvolvida por Belitskii [6] e Elphick [29], e na Seção 3.2 provamos propriedades do operador homológico no contexto reversível equivariante. Finalmente, na Seção 3.3 apresentamos o método para a obtenção da forma normal neste contexto.

\subsection{O método de Belitskii}

Começamos com uma breve introdução sobre o método de Belitskii e seguimos [21]. Nos referimos a [35, 37] para mais detalhes. Remetemos ao Capítulo 1 para relembrar o conceito de germes de aplicações. Seja $h \in \overrightarrow{\mathcal{E}}_{V} \operatorname{com} h(0)=0$, e considere

$$
\dot{x}=h(x), x \in V, 0 \text {. }
$$

Nossos resultados são voltados para uma dedução algébrica para a forma normal de (3.1) que é o primeiro passo no estudo da dinâmica do sistema. O método consiste de sucessivas mudanças de coordenadas na fonte da forma $I+\xi_{k}$ onde $I$ é o germe da identidade e $\xi_{k}$ é um germe de polinômio homogêneo de grau $k$.

A mudança de coordenadas $x=\xi(y)=y+\xi_{k}(y)$ transforma o campo de vetores $h$ no campo conjugado

$$
g(y)=(d \xi)_{x}^{-1} h(\xi(y))
$$

do sistema

$$
\dot{y}=g(y), y \in V, 0 \text {. }
$$

Observamos que

$$
(d \xi)_{x}^{-1}=\left(I+\left(d \xi_{k}\right)_{x}\right)^{-1}=I-\left(d \xi_{k}\right)_{x}+\varphi\left(\left(d \xi_{k}\right)_{x}\right), k \geq 2,
$$

onde $\varphi\left(\left(d \xi_{k}\right)_{x}\right)$ é polinomial e tem grau maior ou igual a $2(k-1)$.

Uma mudança de coordenada do tipo $I+\xi_{k}$ fixa a parte linear $L$, simplifica os termos de ordem $k$ sem alterar os termos de ordem menor ou igual a $k$ e altera termos de ordem maior do que $k$. Note que para cada $k \geq 2, I+\xi_{k}$ é um difeomorfismo numa vizinhança da origem. Ressaltamos aqui que não estamos assumindo que as aplicações sejam analíticas, de forma que o sistema obtido após as mudanças de coordenadas é formalmente conjugado ao sistema original no sentido de que as suas correspondentes séries de Taylor são conjugadas entre si. 
Seja $L$ a linearização de $h$ na origem e considere a expansão em Taylor de $h$ em torno deste ponto

$$
L(x)+h_{2}(x)+h_{3}(x)+\ldots
$$

$\operatorname{com} h_{k} \in \mathcal{P}_{V}^{k}$, para $k \geq 2$.

Fazendo mudanças de coordenadas até grau $k$ em (3.4) e denotando as novas coordenadas novamente por $x$, obtemos

$$
\dot{x}=L x+\sum_{n=2}^{k-1} g_{n}(x)+\tilde{h}_{k}(x)-\left(\left(d \xi_{k}\right)_{(x)} L x-L \xi_{k}(x)\right)+O|x|^{k+1} .
$$

Observe que $\tilde{h}_{k}(x)-\left(\left(d \xi_{k}\right)_{(x)} L x-L \xi_{k}(x)\right)$ é o termo de ordem $k$ de 3.5 .

Agora consideremos o operador homológico $A d_{L}: \overrightarrow{\mathcal{P}}_{V} \rightarrow \overrightarrow{\mathcal{P}}_{V}$ definido por

$$
A d_{L}(p)(x)=(d p)_{(x)} L x-L p(x)
$$

Como $\overrightarrow{\mathcal{P}}_{V}$ é uma álgebra graduada, consideramos o operador $A d_{L}^{k}$ o qual é definido como a restrição de $A d_{L}$ a $\overrightarrow{\mathcal{P}}_{V}^{k}$ já que $A d_{L}$ é linear e preserva a graduação da álgebra $\overrightarrow{\mathcal{P}}_{V}$. Observa-se em (3.5) que o novo sistema pode ser mais simples se os seus termos de grau $k$ são da forma $g_{k}=\tilde{h}_{k}-A d_{L}^{k}\left(\xi_{k}\right)$, para algum $\xi^{k} \in \mathcal{P}_{V}^{k}$, ou seja $g_{k} \in\left(\operatorname{Im} A d_{L}^{k}\right)^{c}$ para algum complementar apropriado.

Obviamente a escolha desse complementar não é única e uma escolha proposta por Belitskii [6] consiste em considerar $\left(\operatorname{Im} A d_{L}^{k}\right)^{c}=\operatorname{ker} A d_{L^{t}}^{k}$ onde $L^{t}$ é a transposta de $L$ :

$$
\overrightarrow{\mathcal{P}}_{V}^{k}=\operatorname{ker} A d_{L^{t}}^{k} \oplus \operatorname{Im} A d_{L}^{k}
$$

Assim, o método consiste em determinar soluções polinomiais $g_{k}$ para $A d_{L^{t}}^{k}=0$. Segue então que o sistema (3.1) é formalmente conjugado a

$$
\dot{x}=L x+g_{2}(x)+g_{3}(x)+\ldots
$$

onde $g_{k} \in \operatorname{ker} A d_{L^{t}}^{k}, k \geq 2$.

Em geral, não é fácil determinar ker $A d_{L^{t}}^{k}$ uma vez que isso envolve resolver uma EDP. Um método alternativo para determinar um complemento foi proposto por Elphick et al. em [29] reconhecendo um grupo de simetrias. Mais precisamente, a partir da linearização $L$, considere

$$
\mathbf{S}=\overline{\left\{e^{s L^{t}}, s \in \mathbb{R}\right\}}
$$


S é um grupo fechado com ação natural em $V$ dada pelo produto de matrizes por vetor. O grupo $\mathbf{S}$ efetivamente introduz simetrias no problema. De fato, os autores provam que

$$
\operatorname{ker} A d_{L^{t}}^{k}=\overrightarrow{\mathcal{P}}_{V}^{k}(\mathbf{S})
$$

e, portanto,

$$
\overrightarrow{\mathcal{P}}_{V}^{k}=\overrightarrow{\mathcal{P}}_{V}^{k}(\mathbf{S}) \oplus A d_{L}^{k}\left(\overrightarrow{\mathcal{P}}_{V}^{k}\right)
$$

(Veja [29, Teorema 2]).

Desta maneira, o método de Elphick permite usar ferramentas da teoria invariante para se calcular a forma normal.

Como veremos no Capítulo 5, quando o interesse é estudar bifurcação de Hopf, por exemplo, $L$ tem autovalores puramente imaginários. Neste caso, o grupo $\mathbf{S}$ é um produto cartesiano de $k$ toros, onde $k$ é o número de autovalores algebricamente independentes da matriz $L$.

\subsection{Propriedades do operador homológico}

No restante deste capítulo consideramos o sistema (3.1), com $h \in \overrightarrow{\mathcal{F}}_{V}(\Gamma)$ e $h(0)=0$ para um grupo de Lie compacto $\Gamma$. Assim, $L=(d h)_{0}$ é $\Gamma$-reversível-equivariante. Os próximos três lemas são úteis para o restante do capítulo.

Lema 3.2.1. O operador homológico é $\Gamma$-reversível-equivariante segundo a ação de $\Gamma$ em $\overrightarrow{\mathcal{P}}_{V}$ definida em 1.10 .

Demonstração: Devemos provar que $\sigma(\gamma) \gamma \star\left(A d_{L}(p)\right)=A d_{L}(\gamma \star p)$. De fato,

$$
\begin{aligned}
\sigma(\gamma) \gamma \star\left(A d_{L}(p)\right)(x) & =\sigma(\gamma) \gamma^{-1}\left((d p)_{(\gamma x)} L(\gamma x)-L p(\gamma x)\right) \\
& =\sigma(\gamma)\left(\gamma^{-1}(d p)_{(\gamma x)} \sigma(\gamma) \gamma L(x)-\gamma^{-1} L p(\gamma x)\right) \\
& =\sigma(\gamma)\left(\sigma(\gamma) \gamma^{-1}(d p)_{(\gamma x)} \gamma L(x)-\sigma\left(\gamma^{-1}\right) L \gamma^{-1} p(\gamma x)\right) \\
& =\gamma^{-1}(d p)_{(\gamma x)} \gamma L(x)-L \gamma^{-1} p(\gamma x)=A d_{L}(\gamma \star p)(x)
\end{aligned}
$$

Lema 3.2.2. O operador homológico $A d_{L}$ permuta as parcelas da decomposição

$$
\overrightarrow{\mathcal{P}}_{V}\left(\Gamma_{+}\right)=\overrightarrow{\mathcal{P}}_{V}(\Gamma) \oplus \overrightarrow{\mathcal{Q}}_{V}(\Gamma)
$$


Demonstração: Se $g(\gamma x)=\gamma g(x)$, temos

$$
\begin{aligned}
A d_{L}(g)(\gamma x) & =(d g)_{(\gamma x)} L(\gamma x)-L g(\gamma x)=\gamma(d g)_{(x)} \gamma^{-1} \sigma(\gamma) \gamma L(x)-\sigma(\gamma) \gamma L g(x) \\
& =\sigma(\gamma) \gamma\left((d g)_{(x)} L-L g(x)\right)=\sigma(\gamma) \gamma A d_{L}(g)(x)
\end{aligned}
$$

e, então $A d_{L}(g) \in \overrightarrow{\mathcal{Q}}_{V}(\Gamma)$. Para provar a próxima afirmação lembramos que $\sigma^{2}(\gamma)=1$ $\forall \gamma \in \Gamma$. Se $g(\gamma x)=\sigma(\gamma) \gamma g(x)$ temos

$$
\begin{aligned}
A d_{L} g(\gamma x) & =(d g)_{(\gamma x)} L(\gamma x)-L g(\gamma x)=\sigma(\gamma) \gamma(d g)_{(x)} \gamma^{-1} \sigma(\gamma) \gamma L(x)-L(\sigma(\gamma) \gamma g(x)) \\
& =\gamma\left((d g)_{(x)} L-L g(x)\right)=\gamma A d_{L} g(x)
\end{aligned}
$$

Da decomposição em (3.11) obtemos

$$
A d_{L}\left(\overrightarrow{\mathcal{P}}_{V}\left(\Gamma_{+}\right)\right)=A d_{L}\left(\overrightarrow{\mathcal{P}}_{V}(\Gamma)\right) \oplus A d_{L}\left(\overrightarrow{\mathcal{Q}}_{V}(\Gamma)\right)
$$

A soma ser direta segue do Lema 3.2.2. Segue também do Lema 3.2 .2 que $\operatorname{Ad}_{L}\left(\overrightarrow{\mathcal{P}}_{V}\left(\Gamma_{+}\right) \subset\right.$ $\overrightarrow{\mathcal{P}}_{V}\left(\Gamma_{+}\right)$. Assim, é possível aplicar os operadores de Reynolds $\vec{R}$ e $\vec{S}$ na decomposição em (3.12). Nesta direção, obtemos o seguinte resultado:

Lema 3.2.3. Os operadores de Reynolds, $\vec{R}$ e $\vec{S}$, satisfazem as igualdades

$$
\vec{R}\left(A d_{L}\left(\overrightarrow{\mathcal{P}}_{V}\left(\Gamma_{+}\right)\right)\right)=A d_{L}\left(\overrightarrow{\mathcal{Q}}_{V}(\Gamma)\right) \quad e \quad \vec{S}\left(A d_{L}\left(\overrightarrow{\mathcal{P}}_{V}\left(\Gamma_{+}\right)\right)\right)=A d_{L}\left(\overrightarrow{\mathcal{P}}_{V}(\Gamma)\right)
$$

Demonstração: Seja $p \in \overrightarrow{\mathcal{P}}_{V}\left(\Gamma_{+}\right)$. Pela definição temos:

$$
\vec{R}\left(A d_{L}(p)\right)=\frac{1}{2}\left(A d_{L}(p)+\delta \star A d_{L}(p)\right) \quad \text { e } \quad \vec{S}\left(A d_{L}(p)\right)=\frac{1}{2}\left(A d_{L}(p)-\delta \star A d_{L}(p)\right) .
$$

Pelo Lema 3.2.1, obtemos

$$
\begin{aligned}
\vec{R}\left(A d_{L}(p)\right) & =\frac{1}{2}\left(A d_{L}(p)-A d_{L}(\delta \star p)\right) \\
& =\frac{1}{2}((d p) L-L p-(d \delta \star p) L+L \delta \star p) \\
& =\left(d \frac{1}{2}(p-\delta \star p)\right) L-L \frac{1}{2}(p-\delta p) \\
& =(d \vec{S}(p)) L-L \vec{S}(p)=A d_{L}(\vec{S}(p))
\end{aligned}
$$


$\mathrm{e}$

$$
\begin{aligned}
\vec{S}\left(A d_{L}(p)\right) & =\frac{1}{2}\left(A d_{L}(p)+A d_{L}(\delta \star p)\right) \\
& =\frac{1}{2}((d p) L-L p+(d \delta \star p) L-L \delta \star p) \\
& =\left(d \frac{1}{2}(p+\delta \star p) L-L \frac{1}{2}(p+\delta \star p)\right. \\
& =(d \vec{R}(p)) L-L \vec{R}(p)=A d_{L}(\vec{R}(p)) .
\end{aligned}
$$

Mas de 1.12 temos $\vec{R}\left(\overrightarrow{\mathcal{P}}_{V}\left(\Gamma_{+}\right)\right)=\overrightarrow{\mathcal{P}}_{V}(\Gamma)$ e $\vec{S}\left(\overrightarrow{\mathcal{P}}_{V}\left(\Gamma_{+}\right)\right)=\overrightarrow{\mathcal{Q}}_{V}(\Gamma)$, e o resultado segue.

\subsection{Forma normal reversível equivariante}

Nesta seção, apresentamos um método algébrico para a obtenção da forma normal de (3.1) com $h \in \overrightarrow{\mathcal{F}}_{V}(\Gamma)$ e $h(0)=0$. Como mencionado antes, isto pode ser feito primeiro resolvendo uma equação diferencial parcial, e depois impondo a existência das simetrias e antissimetrias na forma normal. O resultado que apresentamos aqui fornece um método alternativo, baseado no conhecimento da teoria invariante para $\Gamma$ e $\mathbf{S}$. Exibimos um espaço complementar para o operador homológico $A d_{L}^{k}$ restrito às aplicações $\Gamma$-equivariantes dentro do espaço vetorial das aplicações $\Gamma$-reversíveis-equivariantes. Nosso resultado é reconhecer $\overrightarrow{\mathcal{Q}}_{V}^{k}(\mathbf{S} \rtimes \Gamma)$ como este complemento. Enfatizamos que quando o grupo $\mathbf{S}$ não é compacto, a teoria desenvolvida em [2] ainda pode ser aplicada, desde que exista conjuntos geradores finitos para o anel $\mathcal{P}_{V}(\mathbf{S})$ e para o módulo $\overrightarrow{\mathcal{P}}_{V}(\mathbf{S})$ sobre $\mathcal{P}_{V}(\mathbf{S})$.

Considere o produto semi-direto $\mathbf{S} \rtimes \Gamma$ dos grupo $\mathbf{S}$ e $\Gamma$ onde $\mu: \Gamma \rightarrow \operatorname{Aut}(\mathbf{S})$ de (2.1) é definido por

$$
\mu(\gamma)\left(e^{s L^{t}}\right)=\gamma e^{s L^{t}} \gamma^{-1}
$$

o que corresponde a dizer que $\mu(\gamma)\left(e^{s L^{t}}\right)=e^{\sigma(\gamma) s L^{t}}$. De fato, como $L$ é $\Gamma$-reversívelequivariante e $\gamma^{t}=\gamma^{-1}$, segue que $L^{t}$ também é $\Gamma$-reversível-equivariante e então $\gamma L^{t} \gamma^{-1}=$ $\sigma(\gamma) L^{t}$. Logo, $\gamma e^{L^{t}} \gamma^{-1}=e^{\gamma L^{t} \gamma^{-1}}=e^{\sigma(\gamma) L^{t}}$.

Pela Proposição 2.1.1, este homomorfismo define uma ação de $\mathbf{S} \rtimes \Gamma$ em $V$ :

$$
\left(e^{s L^{t}}, \gamma\right) \cdot v=e^{s L^{t}}(\gamma v)
$$

Neste caso, a igualdade (2.4) corresponde a

$$
e^{s L^{t}}(\gamma v)=\gamma\left(e^{\sigma(\gamma) s L^{t}} v\right)
$$


Teorema 3.3.1. Seja $\Gamma$ um grupo de Lie compacto agindo em $V, \boldsymbol{S}$ definido como em (3.8) e L linear $\Gamma$-reversível-equivariante. Então, para cada $k \geq 2$,

$$
\overrightarrow{\mathcal{Q}}_{V}^{k}(\Gamma)=\overrightarrow{\mathcal{Q}}_{V}^{k}(\boldsymbol{S} \rtimes \Gamma) \oplus A d_{L}^{k}\left(\overrightarrow{\mathcal{P}}_{V}^{k}(\Gamma)\right)
$$

Para a prova do Teorema 3.3.1 apresentamos três lemas. Primeiro, usamos a ação de $\Gamma$ em $\overrightarrow{\mathcal{P}}_{V}$, definida em 1.10, para definir a aplicação $\pi: \overrightarrow{\mathcal{P}}_{V} \rightarrow \overrightarrow{\mathcal{P}}_{V}$ :

$$
\pi(p)=\frac{1}{2}\left(\int_{\Gamma_{+}} \tau \star p d \tau-\int_{\Gamma_{+}}(\delta \tau) \star p d \tau\right),
$$

onde $\int_{\Gamma_{+}}$é a integral de Haar normalizada sobre $\Gamma_{+}$e $\delta$ é uma antissimetria fixada. Note que $\pi$ é uma extensão do operador $\vec{S}$. Mais que isso, eles têm a mesma imagem. De fato, temos:

Lema 3.3.2. A aplicação $\pi: \overrightarrow{\mathcal{P}}_{V}^{k} \rightarrow \overrightarrow{\mathcal{Q}}_{V}^{k}(\Gamma)$ é uma projeção linear a qual preserva a graduação de $\overrightarrow{\mathcal{P}}_{V}$.

Demonstração: Pela linearidade da integral de Haar e da ação de $\Gamma$ segue que $\pi$ é linear e se $p$ tem grau $k$, então o mesmo acontece com $\pi(p)$. Para provar que $\pi(p) \in \overrightarrow{\mathcal{Q}}_{V}^{k}(\Gamma)$, checamos que $\pi(p)=\sigma(\gamma) \gamma \star \pi(p)$ para elementos $\gamma$ em $\Gamma_{+}$e $\delta \Gamma_{+}$separadamente, usando a invariância da integral de Haar à direita e à esquerda e também a normalidade do grupo $\Gamma_{+}$.

Temos,

$$
\begin{aligned}
\sigma(\gamma) \gamma \star(\pi(p)) & =\sigma(\gamma) \gamma \star\left[\frac{1}{2}\left(\int_{\Gamma_{+}} \tau \star(p) d \tau-\int_{\Gamma_{+}}(\delta \tau) \star(p) d \tau\right)\right] \\
& =\frac{1}{2} \sigma(\gamma)\left(\int_{\Gamma_{+}}(\tau \gamma) \star(p) d \tau-\int_{\Gamma_{+}}(\delta \tau \gamma) \star(p) d \tau\right)
\end{aligned}
$$

Se $\gamma \in \Gamma_{+}$então

$$
\begin{aligned}
\sigma(\gamma) \gamma \star \pi(p) & =\frac{1}{2}\left(\int_{\Gamma_{+}}(\tau \gamma) \star p d \tau-\int_{\Gamma_{+}}(\delta \tau \gamma) \star p d \tau\right) \\
& =\frac{1}{2}\left(\int_{\Gamma_{+}} \tau \star p d \tau-\int_{\Gamma_{+}}(\delta \tau) \star p d \tau\right)=\pi(p)
\end{aligned}
$$


Por outro lado, se $\gamma \in \delta \Gamma_{+}$, então $\gamma=\delta \lambda$ para algum $\lambda \in \Gamma_{+}$. Como $\Gamma_{+} \triangleleft \Gamma$ segue que para $\tau \in \Gamma_{+}, \tau \delta=\delta \tilde{\tau}$ para algum $\tilde{\tau} \in \Gamma_{+}$. Assim,

$$
\begin{aligned}
\sigma(\gamma) \gamma \star \pi(p) & =\frac{1}{2}\left(\int_{\Gamma_{+}}-(\tau \gamma) \star p d \tau+\int_{\Gamma_{+}}(\delta \tau \gamma) \star p d \tau\right) \\
& =\frac{1}{2}\left(-\int_{\Gamma_{+}}(\tau \delta \lambda) \star p d \tau+\int_{\Gamma_{+}}(\delta \tau \delta \lambda) \star p d \tau\right) \\
& =\frac{1}{2}\left(-\int_{\Gamma_{+}}(\delta \tilde{\tau} \lambda) \star p d \tilde{\tau}+\int_{\Gamma_{+}}(\overbrace{\delta \delta}^{\delta \Gamma_{+}} \tilde{\tau} \lambda) \star p d \tilde{\tau}\right) \\
& =\frac{1}{2}\left(-\int_{\Gamma_{+}}(\delta \tilde{\tau} \lambda) \star p d \tilde{\tau}+\int_{\Gamma_{+}}(\tilde{\tau} \lambda) \star p d \tilde{\tau}\right) \\
& =\frac{1}{2}\left(\int_{\Gamma_{+}} \tilde{\tau} \star p d \tilde{\tau}-\int_{\Gamma_{+}}(\delta \tilde{\tau}) \star p d \tilde{\tau}\right)=\pi(p) .
\end{aligned}
$$

Logo,

$$
\begin{aligned}
\pi^{2}(p) & =\pi(\pi(p))=\frac{1}{2}\left(\int_{\Gamma_{+}} \tau \star(\pi(p)) d \tau-\int_{\Gamma_{+}}(\delta \tau) \star(\pi(p)) d \tau\right) \\
& =\frac{1}{2}\left(\int_{\Gamma_{+}} \pi(p) d \tau-\int_{\Gamma_{+}}-\pi(p) d \tau\right)=\frac{\pi(p)}{2}\left(\int_{\Gamma_{+}} d \tau-\int_{\Gamma_{+}}-d \tau\right)=\pi(p) .
\end{aligned}
$$

Lema 3.3.3. A projeção $\pi$ definida em (3.17) satisfaz a igualdade $\pi\left(\overrightarrow{\mathcal{P}}_{V}^{k}(\boldsymbol{S})\right)=\overrightarrow{\mathcal{Q}}_{V}^{k}(\boldsymbol{S} \rtimes \Gamma)$.

Demonstração: Se $p \in \overrightarrow{\mathcal{P}}_{V}^{k}(\mathbf{S})$, então $\left(e^{s L^{t}}\right) \star p=p$. Assim, $\pi(p) \in \overrightarrow{\mathcal{Q}}_{V}^{k}(\mathbf{S} \rtimes \Gamma)$ se $\pi(p)=\sigma(\gamma)\left(\gamma e^{\sigma(\gamma) s L^{t}}\right) \star \pi(p)$ para todo $\gamma \in \Gamma$. Temos

$$
\begin{aligned}
\sigma(\gamma)\left(\gamma e^{\sigma(\gamma) s L^{t}}\right) \star \pi(p) & =\sigma(\gamma)\left(\gamma e^{\sigma(\gamma) s L^{t}}\right) \star\left[\frac{1}{2}\left(\int_{\Gamma_{+}} \tau \star(p) d \tau-\int_{\Gamma_{+}}(\delta \tau) \star(p) d \tau\right)\right] . \\
& =\frac{1}{2} \sigma(\gamma)\left(\int_{\Gamma_{+}}\left(\tau \gamma e^{\sigma(\gamma) s L^{t}}\right) \star(p) d \tau-\int_{\Gamma_{+}}\left(\delta \tau \gamma e^{\sigma(\gamma) s L^{t}}\right) \star(p) d \tau\right) .
\end{aligned}
$$

Se $\gamma \in \Gamma_{+}$, então

$$
\begin{aligned}
\sigma(\gamma)\left(\gamma e^{\sigma(\gamma) s L^{t}}\right) \star \pi(p) & =\frac{1}{2}\left(\int_{\Gamma_{+}}\left(\tau \gamma e^{s L^{t}}\right) \star(p) d \tau-\int_{\Gamma_{+}}\left(\delta \tau \gamma e^{s L^{t}}\right) \star(p) d \tau\right) \\
& =\frac{1}{2}\left(\int_{\Gamma_{+}}\left(\tau e^{s L^{t}}\right) \star(p) d \tau-\int_{\Gamma_{+}}\left(\delta \tau e^{s L^{t}}\right) \star(p) d \tau\right) \\
& =\frac{1}{2}\left(\int_{\Gamma_{+}}\left(e^{s L^{t}} \tau\right) \star(p) d \tau-\int_{\Gamma_{+}}\left(e^{\sigma(\delta \tau) s L^{t}} \delta \tau\right) \star(p) d \tau\right) \\
& =\frac{1}{2}\left(\int_{\Gamma_{+}} \tau \star(p) d \tau-\int_{\Gamma_{+}}(\delta \tau) \star(p) d \tau\right)=\pi(p) .
\end{aligned}
$$


Agora, se $\gamma \in \delta \Gamma_{+}$temos $\gamma=\delta \lambda$ para algum $\lambda \in \Gamma_{+}$e, como $\Gamma_{+} \triangleleft \Gamma$ segue que $\delta \tau=\tilde{\tau} \delta$ para algum $\tilde{\tau} \in \Gamma_{+}$. Segue que

$$
\begin{aligned}
\sigma(\gamma)\left(\gamma e^{\sigma(\gamma) s L^{t}}\right) \star \pi(p) & =\frac{1}{2}\left(\int_{\Gamma_{+}}(\overbrace{\delta \tau \gamma}^{\in \Gamma_{+}} e^{-s L^{t}}) \star(p) d \tau-\int_{\Gamma_{+}}(\overbrace{\tau \gamma}^{\in \Gamma_{-}} e^{-s L^{t}}) \star(p) d \tau\right) \\
& =\frac{1}{2}\left(\int_{\Gamma_{+}}\left(e^{-\sigma(\delta \tau \gamma) s L^{t}} \delta \tau \gamma\right) \star(p) d \tau-\int_{\Gamma_{+}}\left(e^{-\sigma(\tau \gamma) s L^{t}} \tau \gamma\right) \star(p) d \tau\right) \\
& =\frac{1}{2}\left(\int_{\Gamma_{+}}\left(e^{-s L^{t}} \delta \tau \gamma\right) \star(p) d \tau-\int_{\Gamma_{+}}\left(e^{s L^{t}} \tau \gamma\right) \star(p) d \tau\right) \\
& =\frac{1}{2}\left(\int_{\Gamma_{+}}(\delta \tau \gamma) \star(p) d \tau-\int_{\Gamma_{+}}(\tau \gamma) \star(p) d \tau\right) \\
& =\frac{1}{2}\left(\int_{\Gamma_{+}}(\delta \tau \delta \lambda) \star(p) d \tau-\int_{\Gamma_{+}}(\tau \delta \lambda) \star(p) d \tau\right) \\
& =\frac{1}{2}\left(\int_{\Gamma_{+}}(\delta \delta \tilde{\tau} \lambda) \star(p) d \tilde{\tau}-\int_{\Gamma_{+}}(\delta \tilde{\tau} \lambda) \star(p) d \tilde{\tau}\right) \\
& =\frac{1}{2}\left(\int_{\Gamma_{+}}(\tilde{\tau}) \star(p) d \tilde{\tau}-\int_{\Gamma_{+}}(\delta \tilde{\tau}) \star(p) d \tilde{\tau}\right)=\pi(p) .
\end{aligned}
$$

Para provar a outra inclusão, seja $g \in \overrightarrow{\mathcal{Q}}_{V}^{k}(\mathbf{S} \rtimes \Gamma)$. Em particular, $g \in \overrightarrow{\mathcal{P}}_{V}^{k}(\mathbf{S})$ e $g=\sigma(\gamma) \gamma \star(g) \forall \gamma \in \Gamma$. Logo,

$\pi(g)=\frac{1}{2}\left(\int_{\Gamma_{+}} \tau \star(g) d \tau-\int_{\Gamma_{+}}(\delta \tau) \star(g) d \tau\right)=\frac{1}{2}\left(\int_{\Gamma_{+}} g d \tau+\int_{\Gamma_{+}} g d \tau\right)=\int_{\Gamma_{+}} g d \tau=g$.

Para a prova do próximo lema, observamos que para $k \geq 2$,

$$
\int_{\Gamma} A d_{L}^{k}(\gamma \star p) d \gamma=A d_{L}^{k} \int_{\Gamma}(\gamma \star p) d \gamma
$$

o que segue diretamente da linearidade de $A d_{L}^{k}$.

Lema 3.3.4. A projeção $\pi$ dada por (3.17) satisfaz a igualdade

$$
\pi\left(A d_{L}\left(\overrightarrow{\mathcal{P}}_{V}\right)\right)=A d_{L}\left(\overrightarrow{\mathcal{P}}_{V}(\Gamma)\right)
$$

Demonstração: Seja $p \in \overrightarrow{\mathcal{P}}_{V}$ e considere a igualdade 3.18). Então, 


$$
\begin{aligned}
\pi\left(A d_{L}(p)\right) & =\frac{1}{2}\left(\int_{\Gamma_{+}} \tau \star\left(A d_{L}(p)\right) d \tau-\int_{\Gamma_{+}}(\delta \tau) \star\left(A d_{L}(p)\right) d \tau\right) \\
& =\frac{1}{2}\left(\int_{\Gamma_{+}} \sigma(\tau) A d_{L}(\tau \star p) d \tau-\int_{\Gamma_{+}} \sigma(\delta \tau) A d_{L}((\delta \tau) \star p) d \tau\right) \\
& =\frac{1}{2}\left(\int_{\Gamma_{+}} A d_{L}(\tau \star p) d \tau+\int_{\Gamma_{+}} A d_{L}((\delta \tau) \star p) d \tau\right) \\
& =\frac{1}{2}\left(A d_{L} \int_{\Gamma_{+}} \tau \star p d \tau+A d_{L} \int_{\Gamma_{+}}(\delta \tau) \star p d \tau\right) \\
& =A d_{L}\left(\frac{1}{2}\left(\int_{\Gamma_{+}} \tau \star p d \tau+\int_{\Gamma_{+}}(\delta \tau) \star p d \tau\right)\right) \\
& =A d_{L}\left(\int_{\Gamma} \tau \star p d \tau\right) .
\end{aligned}
$$

A última igualdade usa o Teorema 1.3.1. Agora, $\int_{\Gamma} \tau \star p d \tau \in \overrightarrow{\mathcal{P}}_{V}(\Gamma)$ e qualquer elemento em $\overrightarrow{\mathcal{P}}_{V}(\Gamma)$ pode ser escrito da forma $\int_{\Gamma} \tau \star p d \tau$, para algum $p \in \overrightarrow{\mathcal{P}}_{V}(\Gamma)$.

Demonstração do Teorema 3.3.1: Aplicamos a projeção $\pi$ dada em 3.17 na igualdade (3.10) e agora usamos os Lemas 3.3.2, 3.3.3 e 3.3.4 para obter

$$
\overrightarrow{\mathcal{Q}}_{V}^{k}(\Gamma)=\overrightarrow{\mathcal{Q}}_{V}^{k}(\mathbf{S} \rtimes \Gamma)+A d_{L}^{k}\left(\overrightarrow{\mathcal{P}}_{V}^{k}(\Gamma)\right) .
$$

Pela Proposição 2.1.2, temos $\overrightarrow{\mathcal{Q}}_{V}^{k}(\mathbf{S} \rtimes \Gamma)=\overrightarrow{\mathcal{P}}_{V}^{k}(\mathbf{S}) \cap \overrightarrow{\mathcal{Q}}_{V}^{k}(\Gamma)$, o qual juntamente com (3.10) nos dá

$$
\overrightarrow{\mathcal{Q}}_{V}^{k}(\mathbf{S} \rtimes \Gamma) \cap A d_{L}^{k}\left(\overrightarrow{\mathcal{P}}_{V}^{k}(\Gamma)\right) \subset \overrightarrow{\mathcal{P}}_{V}^{k}(\mathbf{S}) \cap A d_{L}\left(\overrightarrow{\mathcal{P}}_{V}^{k}\right)=\{0\}
$$

Isto é, a soma em 3.19) é direta, completando assim a prova do Teorema 3.3.1.

De toda a discussão desta seção, o seguinte resultado é uma consequência direta do Teorema 3.3.1.

Teorema 3.3.5. Seja $\Gamma$ um grupo de Lie compacto agindo linearmente em $V$ e seja $h \in \overrightarrow{\mathcal{F}}_{V}(\Gamma)$, com $h(0)=0$ e $L=(d h)_{0}$. Então, o sistema 3.1) é formalmente conjugado a

$$
\dot{x}=L x+g_{2}(x)+g_{3}(x)+\ldots
$$

onde, para cada $k \geq 2, \quad g_{k} \in \overrightarrow{\mathcal{Q}}_{V}^{k}(\boldsymbol{S} \rtimes \Gamma)$. 
Na prática, podemos exibir uma forma geral dos elementos em $\overrightarrow{\mathcal{Q}}_{V}(\mathbf{S} \rtimes \Gamma)$. As principais ferramentas para isto são o Algoritmo 1.4.1 e o Teorema 1.4.2. A partir daí, basta escolher uma aplicação polinomial geral neste módulo até o grau que se deseja truncar a forma normal.

No Capítulo 5 apresentamos exemplos de cálculo de formas normais reversíveis equivariantes pela ação de um grupo do tipo $\mathbf{Z}_{2} \times \mathbf{Z}_{2}$. 



$\frac{m}{4}$

\section{A forma normal de Belitskii-Elphick e o método da transversal completa}

Em teoria de singularidades também há o interesse de escrevermos germes de aplicações em suas formas normais. Esse processo se dá através de uma relação de equivalência, em geral, dada por mudanças de coordenadas na fonte e na meta. A busca por estas formas é conhecida como classificação de germes de aplicações e tem sempre despertado o interesse de muitos pesquisadores. Desta maneira, autores têm desenvolvido ferramentas algébricas que permitem fazer algum tipo de classificação sem precisar conhecer as mudanças de coordenadas. Dentre centenas de trabalhos nesta linha, citamos por exemplo os clássicos trabalhos de Bruce et al. [10], Gaffney e du Plessis [30], Gaffney [31], Wall [59, 60], e em classificação de singularidades aplicada à teoria de bifurcação citamos Golubitsky et al. [34, 35] e Melbourne [48, 49], estes nos contextos com e sem simetrias.

Mas, foi somente em [11] que os autores desenvolvem o chamado método da transversal completa, ferramenta importante para a classificação de germes de aplicações. Mais tarde, Kirk [40] desenvolve um pacote no programa Maple - Transversal - que implementa este 
44 Capítulo 4-A forma normal de Belitskii-Elphick e o método da transversal completa

método. Em [19] Castro e du Plessis comentam sobre o método da transversal completa para germes de aplicações no contexto equivariante.

O objetivo deste capítulo é apresentar nossa percepção de que a forma normal que tratamos nesta tese está estritamente relacionada ao método da transversal completa. A motivação para esta análise se deu primeiramente no caso $\Gamma$-reversível-equivariante, que é o contexto central a que se dedicam os resultados desta tese. Durante o estudo, entretanto, notamos a validade dos resultados mesmo no contexto sem simetrias. Assim, trazemos na Seção 4.2 o estudo no caso sem simetrias e na Seção 4.3 o estudo no caso reversível equivariante. Na Seção 4.1 apresentamos conceitos básicos da teoria de singularidades.

\subsection{Preliminares}

Nesta seção, apresentamos resultados básicos da teoria de singularidades para o desenvolvimento deste capítulo. As referências seguidas aqui são [34, 58].

$\mathrm{O}$ anel $\mathcal{E}_{V}$ é um anel local comutativo e seu ideal maximal é dado por

$$
\mathcal{M}=\left\{f \in \mathcal{E}_{V}, f(0)=0\right\},
$$

onde $V$ é um espaço vetorial real de dimensão finita.

Seja $\mathcal{M}^{k}$ a $k$-ésima potência de $\mathcal{M}$, ou ainda, o espaço dos germes de funções cujos polinômios de Taylor de ordem $k$ na origem são identicamente nulos.

Para $h \in \mathcal{M} \overrightarrow{\mathcal{E}}_{V}$, chamamos o $k$-jato de $h$, e denotamos por $j^{k} h$, o polinômio de Taylor de grau $k$ em 0 de $h(x+a)-h(a)$. Denotamos por $J^{k}$ o espaço dos $k$-jatos dos germes de aplicações polinomiais $g: V, 0 \rightarrow V$ onde cada componente de $g$ é um polinômio de grau menor ou igual a $k$ e $g(0)=0$.

Observação 4.1.1. O espaço $J^{k}$ é isomorfo a um quociente de $\mathcal{E}_{V}$-módulos:

$$
J^{k} \cong \frac{\mathcal{M} \overrightarrow{\mathcal{E}}_{V}}{\mathcal{M}^{k+1} \overrightarrow{\mathcal{E}}_{V}} .
$$

Os métodos tratados aqui para a classificação de germes através de uma relação de equivalência são feitos grau a grau na expansão em série de Taylor do germe a ser estudado. Por isso, uma das principais ideias é substituir o espaço dos germes $\mathcal{M} \overrightarrow{\mathcal{E}}_{V}$ pelo 
espaço dos k-jatos $J^{k}$, para cada k. Isto fica evidenciado em detalhes na Seção 4.2. Lembramos que, nesta tese não estamos trabalhando com aplicações necessariamente analíticas e, neste caso, o sistema obtido após as mudanças de coordenadas é formalmente conjugado ao sistema original, ou seja, as suas correspondentes séries de Taylor são conjugadas entre si.

Agora introduzimos a relação de equivalência em questão seguindo a notação usada por Golubitsky et al. [34, Capítulos II e III].

Definição 4.1.2. Sejam $g$ e $h$ germes de aplicações em $\overrightarrow{\mathcal{E}}_{V}$. Dizemos que $g$ e $h$ são equivalentes se existe um germe de difeomorfismo $X \in \mathcal{M} \overrightarrow{\mathcal{E}}_{V}$ e um germe $S: V, 0 \rightarrow$ $\mathbf{G L}(V)$ tal que

$$
g(x)=S(x) h(X(x))
$$

Vamos denotar por $\tilde{\mathcal{K}}$ o conjunto das equivalências

$$
\tilde{\mathcal{K}}=\left\{(S, X): X \in \mathcal{M} \overrightarrow{\mathcal{E}}_{V} \text { germe de difeomorfismo e } S: V, 0 \rightarrow \mathbf{G L}(V)\right\} .
$$

É definido em $\tilde{\mathcal{K}}$ uma operação que lhe dá estrutura de um produto semi-direto (ver [3]), sendo também um subgrupo geométrico do grupo de contato geral segundo a formulação de Damon [23]. A ação de $\tilde{\mathcal{K}}$ em $\overrightarrow{\mathcal{E}}_{V}$, fica, então,

$$
((S, X) \cdot h)(x):=S(x) h(X(x)) .
$$

Neste caso, a órbita de $h$ segundo a ação de $\tilde{\mathcal{K}}$ é dada por

$$
\tilde{\mathcal{K}} \cdot h=\{(S, X) \cdot h,(S, X) \in \tilde{\mathcal{K}}\}
$$

e o espaço tangente $T \tilde{\mathcal{K}} \cdot h$ à orbita de $h$ é definido como o espaço dos elementos da forma

$$
\frac{d}{d t} \phi(x, t)_{\mid t=0}
$$

para uma família a um parâmetro $t \in \mathbb{R}, 0, \phi(x, t)=S(x, t) h(X(x, t)) \operatorname{com} S(x, 0)=I$ e $X(x, 0)=x$. Um cálculo direto nos dá

$$
T \tilde{\mathcal{K}} \cdot h=\left\{S h+(d h) X, S, X \in \overrightarrow{\mathcal{E}}_{V}\right\}
$$

Um desdobramento a $s$-parâmetros de $g \in \overrightarrow{\mathcal{E}}_{V}$ é um germe $G: V \times \mathbb{R}^{s}, 0 \rightarrow V$ tal que

$$
G(x, 0)=g(x)
$$


46 Capítulo 4-A forma normal de Belitskii-Elphick e o método da transversal completa

Estabelecemos agora uma relação entre um desdobramento $G$ a $s$-parâmetros e um desdobramento $H$ a $l$-parâmetros. Denotamos $\alpha=\left(\alpha_{1}, \ldots, \alpha_{l}\right) \in \mathbb{R}^{l}$ e $\beta=\left(\beta_{1}, \ldots, \beta_{s}\right) \in$ $\mathbb{R}^{s}$.

Definição 4.1.3. Sejam $G$ e $H$ desdobramentos de $h$ com parâmetros $\alpha \in \mathbb{R}^{l}$ e $\beta \in \mathbb{R}^{s}$, respectivamente. Dizemos que $G$ se fatora através de $H$ se existem germes de aplicações $C^{\infty}, S: V \times \mathbb{R}^{s}, 0 \rightarrow \mathbf{G L}(V), \quad X: V \times \mathbb{R}^{s}, 0 \rightarrow V$ e $A: \mathbb{R}^{s}, 0 \rightarrow \mathbb{R}^{l}$ tal que

$$
G(x, \beta)=S(x, \beta) H(X(x, \beta), A(\beta)),
$$

onde, $S(x, 0)=I, \quad X(x, 0)=x \quad$ e $A(0)=0$.

Definição 4.1.4. Um desdobramento $H$ de $h$ é versal se qualquer outro desdobramento de $h$ se fatora através de $H$. Um desdobramento versal é chamado miniversal se possui um número mínimo de parâmetros e esse número é chamado de codimensão de $g$.

Um desdobramento versal de um germe apresenta todas as possíveis deformações deste germe com a variação do seu parâmetro. O reconhecimento de um desdobramento versal é dado por um resultado algébrico essencial da teoria de singularidades ([34, Teorema 2.3]):

Teorema 4.1.5. (Teorema do desdobramento versal) Seja $h \in \overrightarrow{\mathcal{E}}_{V}$ e seja $H$ um desdobramento de h a l parâmetros. Então $H$ é um desdobramento versal de $h$ se, e somente se,

$$
\overrightarrow{\mathcal{E}}_{V}=T \tilde{\mathcal{K}} \cdot h+\mathbb{R}\left\{\frac{\partial H}{\partial \alpha_{1}}(x, 0), \ldots, \frac{\partial H}{\partial \alpha_{l}}(x, 0)\right\}
$$

Como consequência direta do teorema acima, segue que para determinar um desdobramento miniversal para um germe $h$ basta exibirmos um complemento para $T \tilde{\mathcal{K}} \cdot h$ em $\overrightarrow{\mathcal{E}}_{V}([34$, Corolário 2.5$])$ :

Corolário 4.1.6. Seja $h \in \overrightarrow{\mathcal{E}}_{V}$ e $W \subset \overrightarrow{\mathcal{E}}_{V}$ um subespaço vetorial satisfazendo

$$
\overrightarrow{\mathcal{E}}_{V}=T \tilde{\mathcal{K}} \cdot h \oplus W .
$$

Se $\omega_{1}, \ldots, \omega_{r}$ é uma base para $W$, então

$$
H(x, \alpha)=h(x)+\sum_{j=1}^{r} \alpha_{j} \omega_{j}(x)
$$

é um desdobramento miniversal a r parâmetros de $h$.

Para um grupo de Lie compacto $\Gamma$, observamos que os resultados apresentados nesta seção são adaptados para o contexto $\Gamma$-equivariante em [35, Capítulo XIV] e para o contexto $\Gamma$-reversível-equivariante em [3]. 


\subsection{O contexto sem simetrias}

Considere os germes de difeomorfismos $\xi: V, 0 \rightarrow V$ do tipo $\xi=I+\tilde{\xi}$, onde $I$ é o germe da identidade e $\tilde{\xi} \in \bigoplus^{l \geq k} \overrightarrow{\mathcal{P}}_{V}^{k}, k \geq 2$. Seja

$$
\mathcal{G}=\left\{\xi=I+\xi_{k}, \quad \tilde{\xi} \in \bigoplus^{l \geq k} \overrightarrow{\mathcal{P}}_{V}^{k}, \quad k \geq 2\right\} .
$$

Consideramos a ação de $\mathcal{G}$ em $\mathcal{M} \overrightarrow{\mathcal{E}}_{V}$ dada por

$$
(\xi \cdot h)(x)=(d \xi)_{\xi(x)}^{-1} h(\xi(x)), \xi \in \mathcal{G}, h \in \mathcal{M} \overrightarrow{\mathcal{E}}_{V}
$$

Por esta equivalência, dois germes $g, h \in \mathcal{M} \overrightarrow{\mathcal{E}}_{V}$ são equivalentes se existe $\xi \in \mathcal{G}$ tal que

$$
g(x)=(d \xi)_{\xi(x)}^{-1} h(\xi(x))
$$

O grupo $\mathcal{G}$ pode ser identificado com o subgrupo de $\tilde{\mathcal{K}}$ dado pelos pares $\left((d \xi)_{x}^{-1}, \xi\right)$, com $\xi \in \mathcal{G}$. O espaço tangente $T \mathcal{G} \cdot h$ à órbita de $h$ é então definido a partir desta identificação. Notemos que a relação (4.9) fica como em (4.2) e coincide com 3.2.

Como mencionamos na Observação 4.1.1, a classificação de germes considera mudanças de coordenadas que lidam grau a grau para a obtenção de uma forma normal. Para isto, vamos denotar por $J^{k} \mathcal{G}$ o grupo dos $k$-jatos de $\mathcal{G}$, ou seja, $\left\{j^{k} \xi, \xi \in \mathcal{G}\right\}$, e considerar a ação de $J^{k} \mathcal{G}$ em $J^{k}$ para cada $k \geq 2$ induzida por (4.8), a saber

$$
j^{k} \xi \cdot\left(j^{k} h\right)(x)=j^{k}(\xi \cdot h)(x), \quad \xi \in \mathcal{G}, \quad h \in \mathcal{M} \overrightarrow{\mathcal{E}}_{V}
$$

O método da transversal completa é aplicado para subgrupos do grupo de contato geral cujos elementos tenham 1-jato igual ao germe da identidade ([11, Proposição 2.2]). O teorema a seguir apresenta o método para a ação de $J^{k} \mathcal{G}$ em $J^{k}$ :

Teorema 4.2.1. (Transversal completa para jatos) Para $k \geq 1$ fixado, sejam $h \in J^{k} e$ $W \subset \overrightarrow{\mathcal{P}}_{V}^{k+1}$ um subespaço satisfazendo

$$
\mathcal{M}^{k+1} \overrightarrow{\mathcal{E}}_{V} \subset W+T \mathcal{G} \cdot h+\mathcal{M}^{k+2} \overrightarrow{\mathcal{E}}_{V}
$$

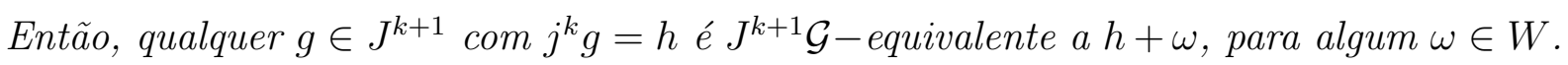


48 Capítulo 4-A forma normal de Belitskii-Elphick e o método da transversal completa

Nosso objetivo agora é apresentar a estreita relação deste método com o resultado de Elphick dado na Seção 3.1

Para $h \in \overrightarrow{\mathcal{E}}_{V}$ vamos definir o operador linear $A d_{h}: \overrightarrow{\mathcal{E}}_{V} \rightarrow \overrightarrow{\mathcal{E}}_{V}$

$$
A d_{h}(\xi)(x)=(d \xi)_{x} h(x)-(d h)_{x} \xi(x)
$$

Consideramos também a restrição $A d_{h}^{k}=\left.A d_{h}\right|_{\overrightarrow{\mathcal{P}}_{V}^{k}}$. Observe que $A d_{h}$ é uma generalização do operador homológico definido em (3.6).

Escrevendo $h$ como $h=L+\tilde{h}, L=(d h)_{0}$, pela linearidade do operador linear segue que

$$
A d_{h}^{k}\left(\xi_{k}\right)=A d_{L}^{k}\left(\xi_{k}\right)+A d_{\tilde{h}}^{k}\left(\xi_{k}\right)
$$

$\xi_{k} \in \overrightarrow{\mathcal{P}}_{V}^{k}$

Assim, seguimos com o primeiro resultado:

Proposição 4.2.2. O espaço tangente à órbita de $h \in \mathcal{M} \overrightarrow{\mathcal{E}}_{V}$ é dado por

$$
T \mathcal{G} \cdot h=\left\{A d_{h}(\tilde{\xi})+\varphi\left(-(d \tilde{\xi})_{x}\right) h, \quad \tilde{\xi} \in \bigoplus^{l \geq k} \overrightarrow{\mathcal{P}}_{V}^{l}, \varphi\left((d \tilde{\xi})_{x}\right) \text { como em (4.13), } k \geq 2\right\} .
$$

Demonstração: Seja $\xi(\cdot, t)$ uma família a 1 parâmetro em $\mathcal{G}, \xi(x, t)=x+\tilde{\xi}(x, t)$, com $\xi(x, 0)=x$. Tomando

$$
\phi(x, t)=(d \xi)_{\xi(x, t)}^{-1} h(\xi(x, t))
$$

todo elemento de $T \mathcal{G} \cdot h$ tem a forma

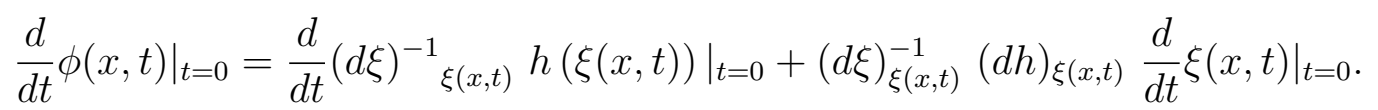

Então,

$$
\begin{aligned}
\frac{d}{d t} \phi(x, 0) & =\frac{d}{d t}(d \xi)_{\xi(x, 0)}^{-1} h(\xi(x, 0))+(d \xi)_{\xi(x, 0)}^{-1}(d h)_{\xi(x, 0)} \frac{d}{d t} \xi(x, 0) \\
& =\frac{d}{d t}(d \xi)_{x}^{-1} h(\xi(x, 0))+(d \xi)_{x}^{-1}(d h)_{x} \frac{d}{d t} \xi(x, 0) \\
& =\frac{d}{d t}(d \xi)_{x}^{-1} h(x)+(d h)_{x} \frac{d}{d t} \xi(x, 0) .
\end{aligned}
$$

$\operatorname{Mas} \frac{d}{d t} \xi(x, 0)=\frac{d}{d t} \tilde{\xi}(x, 0) \quad$ e $\quad(d \xi)_{\xi(x, t)}=I+\left(d \xi_{k}\right)_{\xi(x, t)}$. Semelhante a 3.3) temos

$$
(d \xi)_{\xi(x, t)}^{-1}=I-(d \tilde{\xi})_{\xi(x, t)}+\varphi\left((d \tilde{\xi})_{(x, t)}\right)
$$


com grau de $\varphi\left((d \tilde{\xi})_{(x, t)}\right.$ maior ou igual a $k$.

Logo,

$$
\frac{d}{d t}(d \tilde{\xi})_{\xi(x, t)}^{-1}=-\frac{d}{d t}(d \tilde{\xi})_{\xi(x, t)}+\varphi\left(\frac{d}{d t}(d \tilde{\xi})_{\xi(x, t)}\right)
$$

Portanto,

$$
\frac{d}{d t} \phi(x, 0)=\left(-\frac{d}{d t}(d \tilde{\xi})_{x}+\varphi\left(\frac{d}{d t}(d \tilde{\xi})_{x}\right)\right) h(x)+(d h)_{x} \frac{d}{d t} \tilde{\xi}(x, 0) .
$$

Reescrevendo,

$$
\frac{d}{d t}(d \tilde{\xi})_{x} \equiv(d \tilde{\xi})_{x}, \quad \varphi\left(\frac{d}{d t}(d \tilde{\xi})_{x}\right) \equiv \varphi\left((d \tilde{\xi})_{x}\right) \quad \text { e } \quad \frac{d}{d t} \tilde{\xi}(x, 0) \equiv \tilde{\xi}(x),
$$

segue que todo elemento de $T \mathcal{G} \cdot h$ é da forma

$$
-(d \tilde{\xi})_{x} h(x)+(d h)_{x} \tilde{\xi}(x)+\varphi\left((d \tilde{\xi})_{x}\right) h(x)
$$

$\operatorname{com} \varphi\left((d \tilde{\xi})_{x}\right)$ como em 4.13 de grau maior ou igual a $k$.

Mas $-(d \tilde{\xi})_{x} h(x)+(d h)_{x} \tilde{\xi}(x)=A d_{h}(-\tilde{\xi})(x)$, para $\tilde{\xi} \in \bigoplus^{l \geq k} \overrightarrow{\mathcal{P}}_{V}^{l}$ qualquer. Logo, o resultado segue.

Agora, seja $h \in J^{k}, L=(d h)_{0}$, e considere o grupo $\mathbf{S}$ definido em (3.8). Considere ainda, para cada $k \geq 2$, a decomposição dada em (3.10), a saber,

$$
\overrightarrow{\mathcal{P}}_{V}^{k}=\overrightarrow{\mathcal{P}}_{V}^{k}(\mathbf{S}) \oplus \operatorname{Im} A d_{L}^{k}
$$

Seguimos então com o principal resultado desta seção, que é uma consequência da prova da Proposição 4.2 .2 .

Teorema 4.2.3. Para $k \geq 1$ fixado, seja $h \in J^{k}$ e considere o subespaço $\overrightarrow{\mathcal{P}}_{V}^{k+1}(\boldsymbol{S}) \subset \overrightarrow{\mathcal{P}}_{V}^{k+1}$. Então,

$$
\mathcal{M}^{k+1} \overrightarrow{\mathcal{E}}_{V} \subset \overrightarrow{\mathcal{P}}_{V}^{k+1}(\boldsymbol{S})+T \mathcal{G} \cdot h+\mathcal{M}^{k+2} \overrightarrow{\mathcal{E}}_{V}
$$

Demonstração: Seja $g \in \mathcal{M}^{k+1} \overrightarrow{\mathcal{E}}_{V}$. Consideremos a expansão em Taylor de $g$

$$
g=g_{k+1}+g_{k+2}+g_{k+3}+\ldots
$$

Note que $g_{l} \in \mathcal{M}^{k+2} \overrightarrow{\mathcal{E}}_{V}$ para $l \geq k+2$.

Por 4.15 temos

$$
g_{k+1}=q_{k+1}+p_{k+1},
$$


50 Capítulo 4-A forma normal de Belitskii-Elphick e o método da transversal completa

$\operatorname{com} q_{k+1} \in \overrightarrow{\mathcal{P}}_{V}^{k+1}(\mathbf{S})$ e $p_{k+1} \in \operatorname{Im} A d_{L}^{k+1}$.

Assim, $p_{k+1}=A d_{L}^{k+1}\left(\xi^{k+1}\right)$ para algum $\xi_{k+1} \in \overrightarrow{\mathcal{P}}_{V}^{k+1}$. Para este $\xi_{k+1}$, tome $\varphi\left(-\left(d \xi_{k+1}\right)_{x}\right)$ como em 4.13). Escrevemos $h=L+\tilde{h}$, com $L=(d h)_{0}$ para obter

$$
\begin{aligned}
g_{k+1} & =q_{k+1}+p_{k+1}+A d_{\tilde{h}}\left(\xi_{k+1}\right)+\varphi\left(-\left(d \xi_{k+1}\right)_{x}\right) h-\left(A d_{\tilde{h}}\left(\xi_{k+1}\right)+\varphi\left(-\left(d \xi_{k+1}\right)_{x}\right) h\right) \\
& =q_{k+1}+A d_{L}^{k+1}\left(\xi_{k+1}\right)+A d_{\tilde{h}}\left(\xi_{k+1}\right)+\varphi\left(-\left(d \xi_{k+1}\right)_{x}\right) h-\left(A d_{\tilde{h}}\left(\xi_{k+1}\right)+\varphi\left(-\left(d \xi_{k+1}\right)_{x}\right) h\right) \\
& =q_{k+1}+A d_{h}\left(\xi_{k+1}\right)+\varphi\left(-\left(d \xi_{k+1}\right)_{x}\right) h-\left(A d_{\tilde{h}}\left(\xi_{k+1}\right)+\varphi\left(-\left(d \xi_{k+1}\right)_{x}\right) h\right) .
\end{aligned}
$$

Mas, $q_{k+1} \in \overrightarrow{\mathcal{P}}_{V}^{k+1}(\mathbf{S})$. Ainda, $A d_{h}\left(\xi_{k+1}\right)+\varphi\left(-\left(d \xi_{k+1}\right)_{x}\right) h \in T \mathcal{G} \cdot h$ pela Proposição 4.2.2. Além disso, pela definição do operador linear e $\tilde{h}$, segue também que

$$
A d_{\tilde{h}}\left(\xi_{k+1}\right)+\varphi\left(-\left(d \xi_{k+1}\right)_{x}\right) h \in \mathcal{M}^{k+2} \overrightarrow{\mathcal{E}}_{V}
$$

De fato, $\varphi\left(-\left(d \xi_{k+1}\right)_{x}\right)$ tem grau maior ou igual a $2 k \geq k+2$ pois $k \geq 2$. Logo $g_{k+1} \in \overrightarrow{\mathcal{P}}_{V}^{k}(\mathbf{S})+T \mathcal{G} \cdot h+\mathcal{M}^{k+2} \overrightarrow{\mathcal{E}}_{V}$

Observação 4.2.4. A escolha de um subespaço $W$ que satisfaça as condições do Teorema 4.2.1 não é única. Porém, pela decomposição dada por Elphick 4.15), $\overrightarrow{\mathcal{P}}_{V}^{k}(\boldsymbol{S})$ é o espaço $W$ de menor dimensão que satisfaz o Teorema 4.2.1. Portanto, o reconhecimento da transversal $\overrightarrow{\mathcal{P}}_{V}^{k}(\boldsymbol{S})$ no Teorema 4.2.3 é uma forma alternativa para a obtenção da forma normal de Belitskii-Elphick via o método da transversal completa.

\subsection{O contexto reversível equivariante}

Seja $\Gamma$ um grupo de Lie compacto e considere $\sigma$ definido em 1.4. O objetivo desta seção é adaptar o método da seção anterior no contexto $\Gamma$-reversível-equivariante. Lembramos que se $\sigma$ é trivial então os resultados que apresentamos nesta seção se reduzem ao caso $\Gamma$-equivariante.

Considere as mudanças de coordenadas $\xi: V, 0 \rightarrow V$ do tipo $\xi=I+\tilde{\xi}$, onde $I$ é o germe da identidade e $\tilde{\xi} \in \bigoplus^{l \geq k} \overrightarrow{\mathcal{P}}_{V}^{l}(\Gamma), k \geq 2$. Seja

$$
\tilde{\mathcal{G}}=\left\{\xi=I+\tilde{\xi}, \quad \tilde{\xi} \in \bigoplus^{l \geq k} \overrightarrow{\mathcal{P}}_{V}^{l}(\Gamma), \quad k \geq 2\right\} .
$$

Então $\tilde{\mathcal{G}}$ é um subgrupo de $\mathcal{G}$ e a ação de $\tilde{\mathcal{G}}$ em $\overrightarrow{\mathcal{F}}_{V}(\Gamma)$ é definida como em 4.8). 
Portanto, os germes $g, h \in \overrightarrow{\mathcal{F}}_{V}(\Gamma)$ são $\Gamma$-equivalentes se existe um germe $\xi \in \tilde{\mathcal{G}}$ tal que

$$
g(x)=(d \xi)_{\xi(x)}^{-1} h(\xi(x))
$$

Como o contexto agora é reversível equivariante, as mudanças de coordenadas são feitas grau a grau em aplicações em $\overrightarrow{\mathcal{F}}_{V}(\Gamma)$ que se anulam na origem. Daí, definimos o espaço $J^{k}\left(\Gamma_{\sigma}\right)$ como o espaço dos $k$-jatos $\Gamma$-reversíveis-equivariantes. Observe que se $h \in \overrightarrow{\mathcal{F}}_{V}(\Gamma)$ então $j^{k} h \in J^{k}\left(\Gamma_{\sigma}\right)$ para $k \geq 1$.

Para cada $k \geq 1$ denotamos por $\overrightarrow{\mathcal{F}}_{V k+1}(\Gamma)$ o espaço $\mathcal{M}^{k+1} \overrightarrow{\mathcal{E}}_{V} \cap \overrightarrow{\mathcal{F}}_{V}(\Gamma)$. Como na Observação 4.1.1, a ideia principal neste contexto é trabalharmos com o espaço dos $k$-jatos $J^{k}\left(\Gamma_{\sigma}\right)$ para cada $k$.

Para isto, denotamos por $J^{k} \tilde{\mathcal{G}}$ o grupo dos $k$-jatos de $\tilde{\mathcal{G}}$ para cada $k \geq 1$, ou seja, $\left\{j^{k} \xi, \xi \in \tilde{\mathcal{G}}\right\}$, e consideramos a ação de $J^{k} \tilde{\mathcal{G}}$ em $J^{k}\left(\Gamma_{\sigma}\right)$ induzida por 4.8:

$$
j^{k} \xi \cdot\left(j^{k} h\right)(x)=j^{k}(\xi \cdot h)(x), \quad \xi \in \tilde{\mathcal{G}}, \quad h \in \overrightarrow{\mathcal{F}}_{V}(\Gamma), \quad h(0)=0 .
$$

Em [19], embora sem dar detalhes, Castro e du Plessis sugerem a versão equivariante do Teorema 4.2.1. Verificamos aqui que a versão reversível equivariante para este teorema é direta se considerarmos um subgrupo do grupo de contato geral formado por mudanças de coordenadas $\Gamma$-equivariantes cujos elementos tenham 1 -jato igual ao germe da identidade. Em seguida, interceptamos os espaços no Teorema 4.2.1 com $\overrightarrow{\mathcal{F}}_{V}(\Gamma)$.

Nesta direção, enunciamos a versão reversível equivariante para jatos segundo a ação de $J^{k} \tilde{\mathcal{G}}$ em $J^{k}\left(\Gamma_{\sigma}\right)$ :

Teorema 4.3.1. (Transversal completa para jatos reversivel equivariante) Para $k \geq 1$ fixado, sejam $h \in J^{k}\left(\Gamma_{\sigma}\right)$ e $W \subset \overrightarrow{\mathcal{Q}}_{V}^{k+1}(\Gamma)$ um subespaço satisfazendo

$$
\overrightarrow{\mathcal{F}}_{V k+1}(\Gamma) \subset W+T \tilde{\mathcal{G}} \cdot h+\overrightarrow{\mathcal{F}}_{V k+2}(\Gamma)
$$

Então, qualquer $g \in J^{k+1}\left(\Gamma_{\sigma}\right)$ com $j^{k} g=h$ é $J^{k+1} \tilde{\mathcal{G}}$-equivalente a $h+\omega$, para algum $\omega \in W$.

O que fazemos agora é determinar o espaço tangente $T \tilde{\mathcal{G}} \cdot h$ à órbita de $h \in \overrightarrow{\mathcal{F}}_{V}(\Gamma)$, $h(0)=0$. O processo é análogo ao que fizemos na seção anterior. 
52 Capítulo 4-A forma normal de Belitskii-Elphick e o método da transversal completa

Proposição 4.3.2. O espaço tangente à órbita de $h \in \overrightarrow{\mathcal{F}}_{V}(\Gamma)$ com $h(0)=0$ é dado por

$$
T \tilde{\mathcal{G}} \cdot h=\left\{A d_{h}(\tilde{\xi})+\varphi\left((d \tilde{\xi})_{x}\right) h, \quad \tilde{\xi} \in \bigoplus^{l \geq k} \overrightarrow{\mathcal{P}}_{V}^{l}(\Gamma), \varphi\left((d \tilde{\xi})_{x}\right) \text { como em (4.13), } k \geq 2\right\} .
$$

A demonstração desta proposição é análoga à demonstração da Proposição 4.2.2. Observamos aqui que, neste caso, para cada $x \in V, 0$ a aplicação $\varphi\left((d \tilde{\xi})_{x}\right)$ é $\Gamma$-equivariante.

Seja $h \in J^{k}\left(\Gamma_{\sigma}\right), L=(d h)_{0}$. Considere ainda o grupo $\mathbf{S}$ definido em $(3.8)$ associado a $L$. Apresentamos explicitamente um espaço que satisfaz as condições do Teorema 4.3.1 para a ação do grupo $\tilde{\mathcal{G}}$. Para isto, remetemos à Seção 3.3 para o enunciado a seguir:

Teorema 4.3.3. Para cada $k \geq 1$ fixado, seja $h \in J^{k}\left(\Gamma_{\sigma}\right)$ e considere o subespaço $\overrightarrow{\mathcal{Q}}_{V}^{k+1}(\boldsymbol{S} \rtimes \Gamma) \subset \overrightarrow{\mathcal{Q}}_{V}^{k+1}(\Gamma)$. Então,

$$
\overrightarrow{\mathcal{F}}_{V k+1}(\Gamma) \subset \overrightarrow{\mathcal{Q}}_{V}^{k+1}(\boldsymbol{S} \rtimes \Gamma)+T \tilde{\mathcal{G}} \cdot h+\overrightarrow{\mathcal{F}}_{V k+2}(\Gamma) .
$$

A prova deste teorema segue os passos da demonstração do Teorema 4.2.3. Para tanto, usamos a decomposição

$$
\overrightarrow{\mathcal{Q}}_{V}^{k+1}(\Gamma)=\overrightarrow{\mathcal{Q}}_{V}^{k+1}(\mathbf{S} \rtimes \Gamma) \oplus A d_{L}^{k+1}\left(\overrightarrow{\mathcal{P}}_{V}^{k+1}(\Gamma)\right),
$$

dada pelo Teorema 3.3.1. Em seguida, análogo à condição (4.16) observamos que para $k \geq 2$,

$$
A_{\tilde{h}}\left(\xi_{k+1}\right)+\varphi\left(-\left(d \xi_{k+1}\right)_{x}\right) h \in \overrightarrow{\mathcal{F}}_{V_{k+2}}(\Gamma),
$$

uma vez que $\tilde{h}, h$ são $\Gamma$-reversíveis-equivariantes, $\xi_{k+1},\left(d \xi_{k+1}\right)_{x}$ são $\Gamma$-equivariantes.

Encerramos este capítulo com uma observação:

Observação 4.3.4. O Teorema 4.3.3 é a versão reversivel equivariante para o Teorema 4.2.3. No contexto sem simetrias a transversal completa pode ser escolhida como sendo o espaço $\mathcal{P}_{V}^{k}(\boldsymbol{S})$ e, como vimos na Observação 4.2.4, este espaço é o de menor dimensão. De forma análoga, pela decomposição (4.19), $\overrightarrow{\mathcal{Q}}_{V}^{k}(\boldsymbol{S} \rtimes \Gamma)$ é o espaço $W$ de menor dimensão que satisfaz as condições do Teorema 4.3.1. 


\begin{tabular}{l|l|}
\hline & Capítulo \\
\cline { 2 - 2 } & 5 \\
\hline
\end{tabular}

\section{Campos de vetores}

\section{$\mathbf{Z}_{2} \times \mathbf{Z}_{2}$-reversíveis-equivariantes}

Este capítulo é dedicado a aplicações do método que desenvolvemos no Capítulo 3 para obtenção da forma normal. Calculamos formas normais de campos de vetores cuja linearização na origem tem uma parte nilpotente e uma parte com autovalores puramente imaginários. O interesse do nosso estudo para esta classe de campos de vetores é da sua frequência na teoria de sistemas dinâmicos; em particular, na investigação de ocorrência de órbitas periódicas, ciclos homoclínicos e heteroclínicos em sistemas reversíveis. Neste caso, é viável e facilitador utilizar o método que desenvolvemos no Capítulo 3 , uma vez que, o grupo $\mathbf{S}$ definido em (3.8) tem uma forma bem específica, a qual dependerá da ressonância ou não ressonância do sistema.

Em [44], por exemplo, os autores usam o método de Belitskii para chegar a formas normais para o estudo de ocorrência de famílias de órbitas periódicas de duas classes especiais de sistemas reversíveis, sendo uma ressonante, outra não ressonante. Para o caso ressonante, a forma normal naquele trabalho é truncada em um baixo grau, e os 
cálculos ficam cada vez mais complicados para valores crescentes deste grau. Vamos usar nosso método algébrico neste exemplo, ilustrando como ele fornece de maneira direta a forma normal explicitando sua forma geral. Em consequência, obtemos diretamente a forma normal de grau qualquer que se deseje, ou necessite, truncar a série de Taylor do campo de vetores em questão. Sendo assim, resultados que apresentamos neste capítulo generalizam algumas formas normais obtidas em [44].

Mais especificamente, estamos interessados em deduzir formas normais para sistemas do tipo

$$
\dot{x}=h(x)
$$

$\operatorname{com} h: \mathbb{R}^{2 n+2}, 0 \rightarrow \mathbb{R}^{2 n+2}, h \in C^{\infty}, h(0)=0$ cuja linearização $(d h)_{0}$ na origem tem matriz da forma

$$
L=\left(\begin{array}{ccccccc}
0 & 1 & & & & & \\
0 & 0 & & & & 0 & \\
& & 0 & \omega_{1} & & & \\
& & -\omega_{1} & 0 & & & \\
& & & & \ddots & & \\
& 0 & & & & 0 & \omega_{n} \\
& & & & & -\omega_{n} & 0
\end{array}\right)
$$

para certos valores reais $\omega_{1}, \ldots, \omega_{n}$ maiores que zero tal que $\omega_{i}^{2} \neq \omega_{j}^{2}$ para $i \neq j$ em $\{1, \ldots, n\}$. Assumimos que em (5.1) há duas antissimetrias que são involuções lineares que geram um grupo abeliano. Sob esta consideração, o primeiro passo é investigar quais são os possíveis pares de involuções que anti-comutam com $L$ dada em (5.2). A Seção 5.1 é dedicada à classificação de tais pares.

Na Seção 5.2 descrevemos o processo para o cálculo de formas normais considerando a ação deste grupo gerado pelas involuções. A Seção 5.3 é dedicada para o cálculo da forma explícita das formas normais provenientes das diferentes possibilidades dos pares de involuções obtidos na Seção 5.1, primeiramente sem ressonância e, em seguida, com ressonância. 


\subsection{Pares de involuções que anti-comutam com o campo de vetores}

Uma involução é um germe de difeomorfismo $\varphi: \mathbb{R}^{n}, 0 \rightarrow \mathbb{R}^{n}, 0$ que satisfaz $\varphi \circ \varphi=I$. Denotamos por $\operatorname{Fix}(\varphi)$ o espaço de pontos fixos de $\varphi$,

$$
\operatorname{Fix}(\varphi)=\left\{x \in \mathbb{R}^{n}, 0, \varphi(x)=x\right\}
$$

Definição 5.1.1. Dois pares de involuções $\left(\varphi_{1}, \varphi_{2}\right)$ e $\left(\bar{\varphi}_{1}, \bar{\varphi}_{2}\right)$ em $\mathbb{R}^{n}, 0$ são equivalentes se existe um germe de difeomorfismo $H$ de $\mathbb{R}^{n}, 0$ tal que $\bar{\varphi}_{i}=H \circ \varphi_{i} \circ H^{-1}$ para $i=1,2$.

Sejam $\mathbf{Z}_{2}^{\phi}$ e $\mathbf{Z}_{2}^{\psi}$ os grupos gerados pelas involuções lineares $\phi$ e $\psi$, respectivamente, e seja $\langle\phi, \psi\rangle=\mathbf{Z}_{2}^{\phi} \times \mathbf{Z}_{2}^{\psi}$ para $\phi \neq \psi$.

Como mencionamos no início deste capítulo, começamos pela busca das possíveis involuções $\varphi$ de $\mathbb{R}^{2 n+2}, 0$ tais que

$$
\varphi L=-L \varphi
$$

com $L$ definida em (5.2). Um cálculo direto leva a involução $\varphi$ ter sua forma matricial dada por

$$
\varphi=\left(\begin{array}{ccc}
\varphi_{0} & & 0 \\
& \ddots & \\
0 & & \varphi_{n}
\end{array}\right)
$$

onde

$$
\varphi_{0}=\left(\begin{array}{cc}
a_{0} & 0 \\
0 & -a_{0}
\end{array}\right) \quad \text { e } \quad \varphi_{i}=\left(\begin{array}{cc}
a_{i} & b_{i} \\
b_{i} & -a_{i}
\end{array}\right)
$$

com $a_{0}^{2}=1$ e $a_{i}^{2}+b_{i}^{2}=1$ para $1 \leq i \leq n$. Assim, cada $\varphi_{i}$ é uma reflexão de ordem 2 para $0 \leq i \leq n$. Então $\operatorname{dim} \operatorname{Fix}\left(\varphi_{i}\right)=1$ para $0 \leq i \leq n$ e $\operatorname{dim} \operatorname{Fix}(\varphi)=n+1$.

Sejam $(\phi, \psi)$ e $(\bar{\phi}, \bar{\psi})$ pares de involuções lineares que geram um grupo abeliano com pré-formas normais como em (5.4). Então, $(\phi, \psi)$ e $(\bar{\phi}, \bar{\psi})$ são equivalentes se, e somente se, existe um isomorfismo linear $H$ de ordem $2 n+2$ tal que

$$
\bar{\phi}=H \phi H^{-1} \quad \text { e } \quad \bar{\psi}=H \psi H^{-1}
$$


Como o nosso objetivo é preservar a parte linear do sistema, devemos ter $H L=L H$. Assim, $H$ tem matriz da forma

$$
\left(\begin{array}{ccc}
H_{0} & & 0 \\
& \ddots & \\
0 & & H_{n}
\end{array}\right)
$$

sendo $H_{i}$ matrizes invertíveis de ordem 2 que dão a equivalência entre $\left(\phi_{i}, \psi_{i}\right)$ e $\left(\bar{\phi}_{i}, \bar{\psi}_{i}\right)$ para $0 \leq i \leq n$.

Portanto, a classificação de pares de involuções lineares em $\mathbb{R}^{2 n+2}$ que comutam e satisfazem (5.3) se reduz à classificação de pares de reflexões em $\mathbb{R}^{2}$ que comutam.

Devido às restrições em (5.5) segue que se $\operatorname{Fix}\left(\phi_{i}\right)$ e $\operatorname{Fix}\left(\psi_{i}\right)$ são transversais, $0 \leq i \leq n$, usando [46, Teorema 6.2] temos $\left(\phi_{i}, \psi_{i}\right)$ equivalente ao par

$$
\left(\begin{array}{cc}
1 & 0 \\
0 & -1
\end{array}\right),\left(\begin{array}{cc}
-1 & 0 \\
0 & 1
\end{array}\right)
$$

Caso contrário, se $\operatorname{Fix}\left(\phi_{i}\right)$ e $\operatorname{Fix}\left(\psi_{i}\right)$ não são transversais então eles coincidem e, portanto, $\phi_{i}=\psi_{i}$ para $0 \leq i \leq n$.

Assim, fixando $\phi_{i}=\left(\begin{array}{cc}1 & 0 \\ 0 & -1\end{array}\right)$ para todo $0 \leq i \leq n$, as possibilidades para $\psi_{i}$, $0 \leq i \leq n$, são uma das formas em (5.7).

Então, existem $2^{n}$ pares de involuções $(\phi, \psi)(\operatorname{com} \phi \neq \psi)$ tal que $\langle\phi, \psi\rangle=\mathbf{Z}_{2}^{\phi} \times \mathbf{Z}_{2}^{\psi}$, a saber

$$
\phi=\left(\begin{array}{ccccc}
1 & & & & \\
& -1 & & 0 & \\
& & \ddots & & \\
& 0 & & 1 & \\
& & & & -1
\end{array}\right) \text { e } \psi=\left(\begin{array}{ccccc}
a_{0} & & & & \\
& -a_{0} & & 0 & \\
& & \ddots & & \\
& & & a_{n} & \\
& & & & -a_{n}
\end{array}\right) \text {, }
$$

com $a_{i}= \pm 1$ para todo $0 \leq i \leq n$.

Quando $a_{i}=1,0 \leq i \leq n$ temos $\phi=\psi$. Para este caso, as formas normais determinadas nas próximas seções correspondem às formas normais $\mathbf{Z}_{2}^{\phi}$-reversíveis-equivariantes. 


\subsection{Cálculo da forma normal}

Sejam $\phi$ e $\psi$ involuções lineares num espaço real $V$ de dimensão finita e considere a ação de $\Gamma=\mathbf{Z}_{2}^{\phi} \times \mathbf{Z}_{2}^{\psi}$ em $V$ dada em (2.2). Suponha ainda que $\phi$ e $\psi$ sejam reversibilidades. Neste caso, temos $\Gamma_{+}=\langle\phi \circ \psi\rangle$.

Pelo Algoritmo 1.4 .1 é possível determinar geradores para o módulo $\overrightarrow{\mathcal{Q}}_{V}(\Gamma)$ sobre o anel $\mathcal{P}_{V}(\Gamma)$, uma vez conhecida uma base de Hilbert para o anel $\mathcal{P}_{V}\left(\Gamma_{+}\right)$e fixando uma antissimetria $\delta$ de $\Gamma$. O que mostramos aqui é que este processo pode ser simplificado ainda mais quando o grupo é gerado por um par de involuções que comutam. Para isto, remetemos ao Capítulo 1 e relembramos a definição de representação dual.

Sejam $(\rho, V)$ e $(\eta, V)$ as representações canônicas de $\mathbf{Z}_{2}^{\phi}$ e $\mathbf{Z}_{2}^{\psi}$, respectivamente, e consideremos a representação produto $(\rho \eta, V)$ de $\mathbf{Z}_{2}^{\phi} \times \mathbf{Z}_{2}^{\psi}$ dada em 2.2 .

Agora, consideremos $\sigma_{1}: \mathbf{Z}_{2}^{\phi} \rightarrow \mathbf{Z}_{2}$ e $\sigma_{2}: \mathbf{Z}_{2}^{\psi} \rightarrow \mathbf{Z}_{2}$ os homomorfismos definidos em (1.4), e então $\sigma_{1}(\phi)=\sigma_{2}(\psi)=-1$.

O objetivo é determinar geradores para $\overrightarrow{\mathcal{Q}}_{(\rho \eta, V)}\left(\mathbf{Z}_{2}^{\phi} \times \mathbf{Z}_{2}^{\psi}\right)$ sobre o anel $\mathcal{P}_{(\rho \eta, V)}\left(\mathbf{Z}_{2}^{\phi} \times \mathbf{Z}_{2}^{\psi}\right)$. Para isto, consideremos também a representação dual $\left(\rho_{\sigma_{1}}, V\right)$ de $\mathbf{Z}_{2}^{\phi}$ definida em (1.8).

Como vimos na Seção 1.3 , o caso reversível equivariante é um caso equivariante se tomarmos na meta a representação dual, ou seja,

$$
\overrightarrow{\mathcal{Q}}_{(\rho, V)}\left(\mathbf{Z}_{2}^{\phi}\right)=\overrightarrow{\mathcal{P}}_{(\rho, V),\left(\rho_{\sigma_{1}}, V\right)}\left(\mathbf{Z}_{2}^{\phi}\right)
$$

Portanto, o processo de obtenção dos geradores para $\overrightarrow{\mathcal{Q}}_{(\rho \eta, V)}\left(\mathbf{Z}_{2}^{\phi} \times \mathbf{Z}_{2}^{\psi}\right)$ e de uma base de Hilbert para $\mathcal{P}_{(\rho \eta, V)}\left(\mathbf{Z}_{2}^{\phi} \times \mathbf{Z}_{2}^{\psi}\right)$ é dado por:

1. Tome $\Gamma=\mathbf{Z}_{2}^{\phi}$ e fixe a antissimetria $\delta=\phi$. Então, $\Gamma_{+}=\{I\}$. Tome uma base de Hilbert para $\mathcal{P}_{(\rho, V)}\left(\Gamma_{+}\right)$e um conjunto de geradores para $\overrightarrow{\mathcal{P}}_{(\rho, V)}\left(\Gamma_{+}\right)$.

2. Aplique o Algoritmo 1.4.1 para calcular geradores para $\overrightarrow{\mathcal{Q}}_{(\rho, V)}(\Gamma)$ sobre $\mathcal{P}_{(\rho, V)}(\Gamma)$. Aplique o Teorema 1.4 .2 para calcular uma base de Hilbert para $\mathcal{P}_{(\rho, V)}(\Gamma)$.

3. Tome $\tilde{\Gamma}=\mathbf{Z}_{2}^{\phi} \times \mathbf{Z}_{2}^{\psi}$ e fixe a antissimetria $\delta=\psi$. Considere a representação dual de $\mathbf{Z}_{2}^{\phi}$ em $V$. Então, $\tilde{\Gamma}_{+}=\mathbf{Z}_{2}^{\phi}$. Considere os geradores para $\mathcal{P}_{(\rho, V)}\left(\Gamma_{+}\right)$e $\overrightarrow{\mathcal{P}}_{(\rho, V),\left(\rho_{\sigma_{1}}, V\right)}\left(\Gamma_{+}\right)$ obtidos no item 2. Aplique o Algoritmo 1.4 .1 para obter geradores para $\overrightarrow{\mathcal{Q}}_{(\rho \eta, V)}(\tilde{\Gamma})$. 
4. Considere a base de Hilbert para $\mathcal{P}_{(\rho, V)}\left(\Gamma_{+}\right)$calculada no item 2 e fixe a antissimetria $\delta=\psi$. Aplique o Teorema 1.4 .2 para obter geradores para $\mathcal{P}_{(\rho \eta, V)}(\tilde{\Gamma})$.

O que enfatizamos aqui é que, pelos passos acima, podemos aplicar o Algoritmo 1.4.1 duas vezes de forma conveniente para determinarmos geradores para aplicações $\mathbf{Z}_{2}^{\phi} \times$ $\mathbf{Z}_{2}^{\psi}$-reversíveis-equivariantes. A principal vantagem é que o ponto de partida para a construção destes geradores é determinar geradores para os invariantes e equivariantes pelo grupo $\Gamma_{+}=\{I\}$.

Notamos que este processo se generaliza para uma $s$-upla de involuções lineares que comutam, ou seja, se $\left\langle\phi_{1}, \ldots, \phi_{s}\right\rangle=\mathbf{Z}_{1}^{\phi_{1}} \times \ldots \times \mathbf{Z}_{s}^{\phi_{s}}$, e cada $\phi_{i}$ é uma reversibilidade, então aplicamos o Algoritmo $1.4 .1 s$ vezes para determinar geradores para $\overrightarrow{\mathcal{Q}}_{V}\left(\mathbf{Z}_{1}^{\phi_{1}} \times \ldots \times \mathbf{Z}_{s}^{\phi_{s}}\right)$.

Este processo auxilia de forma significativa o cálculo de formas normais de sistemas reversíveis equivariantes sob a ação de um grupo do tipo $\mathbf{Z}_{2}^{\phi} \times \mathbf{Z}_{2}^{\psi}$. Como vemos a seguir, este cálculo pode ser feito de forma sistemática em alguns passos.

Pelos Teoremas 3.3.1 e 3.3.5, para determinar a forma normal $\mathbf{Z}_{2}^{\phi} \times \mathbf{Z}_{2}^{\psi}$-reversívelequivariante de 5.1$)$ com $L=(d h)_{0}$, é preciso calcular geradores para $\overrightarrow{\mathcal{Q}}_{(\rho \eta, V)}\left(\mathbf{S} \rtimes\left(\mathbf{Z}_{2}^{\phi} \times\right.\right.$ $\left.\mathbf{Z}_{2}^{\psi}\right)$ ) e uma base de Hilbert para o anel $\mathcal{P}_{(\rho \eta, V)}\left(\mathbf{S} \rtimes\left(\mathbf{Z}_{2}^{\phi} \times \mathbf{Z}_{2}^{\psi}\right)\right)$, onde $\mathbf{S}$ é o grupo definido em (3.8). O procedimento que apresentamos a seguir facilita a obtenção destes geradores. A principal vantagem, neste caso, é que o ponto de partida é determinar geradores para os invariantes e equivariantes pela ação de $\mathbf{S}$. Para isto, seguimos os seguintes passos:

1. Tome $\Gamma=\mathbf{S} \rtimes \mathbf{Z}_{2}^{\phi}$ e fixe a antissimetria $\delta=\phi$. Então, $\Gamma_{+}=\mathbf{S}$. Tome uma base de Hilbert para $\mathcal{P}_{V}(\mathbf{S})$ e um conjunto de geradores para $\overrightarrow{\mathcal{P}}_{V}(\mathbf{S})$ como módulo sobre $\mathcal{P}_{V}(\mathbf{S})$.

2. Aplique o Algoritmo 1.4 .1 para obter geradores para $\overrightarrow{\mathcal{Q}}_{(\rho, V)}\left(\mathbf{S} \rtimes \mathbf{Z}_{2}^{\phi}\right)$. Aplique o Teorema 1.4 .2 para obter uma base de Hilbert para $\mathcal{P}_{(\rho, V)}\left(\mathbf{S} \rtimes \mathbf{Z}_{2}^{\phi}\right)$.

3. Tome $\tilde{\Gamma}=\mathbf{S} \rtimes\left(\mathbf{Z}_{2}^{\phi} \times \mathbf{Z}_{2}^{\psi}\right)$ e fixe a antissimetria $\delta=\psi$. Considere a representação dual de $\mathbf{Z}_{2}^{\phi}$ em $V$. Então $\tilde{\Gamma}_{+}=\mathbf{S} \rtimes \mathbf{Z}_{2}^{\phi}$. Considere os geradores para $\mathcal{P}_{(\rho, V)}\left(\tilde{\Gamma}_{+}\right)$e $\overrightarrow{\mathcal{P}}_{(\rho, V),\left(\rho_{\sigma_{1}}, V\right)}\left(\tilde{\Gamma}_{+}\right)$obtidos no item 2. Aplique o Algoritmo 1.4.1 para obter geradores $\operatorname{para} \overrightarrow{\mathcal{Q}}_{(\rho \eta, V)}(\tilde{\Gamma})$.

4. Considere a base de Hilbert para $\mathcal{P}_{(\rho, V)}\left(\tilde{\Gamma}_{+}\right)$calculada no item 2 e fixe a antissimetria $\delta=\psi$. Aplique o Teorema 1.4 .2 para obter uma base de Hilbert para $\mathcal{P}_{(\rho, V)}(\tilde{\Gamma})$. 


\subsection{Formas normais $Z_{2} \times Z_{2}$-reversíveis-equivariantes}

Nesta seção consideramos o sistema (5.1) com $(d h)_{0}$ como em 5.2. Usamos os resultados da Seção 5.2 para calcular formas normais $\mathbf{Z}_{2}^{\phi} \times \mathbf{Z}_{2}^{\psi}$-reversíveis-equivariantes para as diferentes possibilidades de $\phi$ e $\psi$ como em (5.8).

Seja $L=D+N$ a decomposição de Jordan-Chevalley para $L$, onde $D$ é a parte semisimples de $L, N$ é a parte nilpotente de $L$ e $D N=N D$. Suponha que os autovalores não nulos de $L$ sejam puramente imaginários. Então, o grupo $\mathbf{S}$ definido em (3.8) tem uma estrutura particular:

Teorema 5.3.1. [35, Proposição XVI 5.7] Seja $L=D+N$ a decomposição de JordanChevalley de L. Se $N=0$, então $\mathbf{S}=\mathbf{T}^{k}$. Se $N \neq 0$, então $\mathbf{S}=\mathbf{R} \times \mathbf{T}^{k}$, onde $k$ é o número de autovalores algebricamente independentes de $L, \mathbf{T}^{k}=\overline{\left\{e^{s D}, s \in \mathbb{R}\right\}} e$ $\mathbf{R}=\left\{e^{s N^{t}}, s \in \mathbb{R}\right\}$.

\subsubsection{Caso não ressonante}

Nesta subseção obtemos a forma normal de (5.1), com $(d h)_{0}$ definida em $(5.2)$ e $\omega_{1}, \ldots, \omega_{n}$ algebricamente independentes. Neste caso, o sistema (5.1) é chamado de não ressonante.

Considere o grupo S definido em (3.8). Pelo Teorema 5.3.1 temos

$$
\mathbf{S}=\mathbf{R} \times \mathbf{T}^{n}, \quad \text { onde } \mathbf{R} \cong\left\{\left(\begin{array}{ll}
1 & 0 \\
s & 1
\end{array}\right), s \in \mathbb{R}\right\}
$$

Observe que o grupo S é não compacto. Porém, como já falamos no Capítulo 1, podemos ainda aplicar o Algoritmo 1.4.1 uma vez que haja um conjunto finito de geradores para os invariantes e equivariantes pela ação de $\mathbf{S}$.

Usamos aqui coordenadas complexas $(x, z)=\left(x_{1}, x_{2}, z_{1}, \ldots, z_{n}\right) \in \mathbb{R}^{2} \times \mathbb{C}^{n}$. A ação de $\mathbf{S}$ em $\mathbb{R}^{2} \times \mathbb{C}^{n}$ é diagonal e é dada a partir das ações de $\mathbf{R}$ em $\mathbb{R}^{2}$ e $\mathbf{T}^{n}$ em $\mathbb{C}^{n}$, respectivamente:

$$
s\left(x_{1}, x_{2}\right)=\left(x_{1}, s x_{1}+x_{2}\right) \text { e }\left(\theta_{1}, \ldots, \theta_{n}\right)\left(z_{1}, \ldots, z_{n}\right)=\left(e^{i \theta_{1}} z_{1}, \ldots, e^{i \theta_{n}} z_{n}\right),
$$

para $s \in \mathbf{R}$ e $\left(\theta_{1}, \ldots, \theta_{n}\right) \in \mathbf{T}^{n}$. 
As ações de $\mathbf{Z}_{2}^{\phi}$ e $\mathbf{Z}_{2}^{\psi}$ em $\mathbb{R}^{2} \times \mathbb{C}^{n}$ ficam

$$
\phi\left(x_{1}, x_{2}, z_{1}, \ldots, z_{n}\right)=\left(x_{1},-x_{2}, \bar{z}_{1}, \ldots, \bar{z}_{n}\right),
$$

e,

$$
\psi\left(x_{1}, x_{2}, z_{1}, \ldots, z_{n}\right)=\left(a_{0} x_{1},-a_{0} x_{2}, a_{1} \bar{z}_{1}, \ldots, a_{n} \bar{z}_{n}\right) .
$$

Para a ação de $\mathbf{R}$ em $\mathbb{R}^{2}$, um cálculo simples mostra que $\overrightarrow{\mathcal{P}}_{\mathbb{R}^{2}}(\mathbf{R})$ é gerado por

$$
\left\{\left(x_{1}, x_{2}\right),(0,1)\right\}
$$

sobre o anel $\mathcal{P}_{\mathbb{R}^{2}}(\mathbf{R})=\left\langle x_{1}\right\rangle$.

Para $\tilde{\Gamma}=\mathbf{S} \rtimes\left(\mathbf{Z}_{2}^{\phi} \rtimes \mathbf{Z}_{2}^{\psi}\right)$, segue dos Teoremas 3.3.1 e 3.3.5, que precisamos calcular o

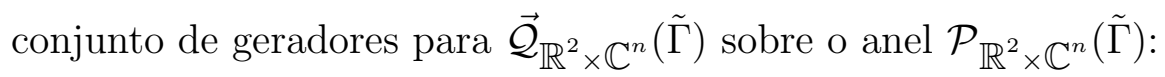

1. Seja $\Gamma=\mathbf{S} \rtimes \mathbf{Z}_{2}^{\phi}$ e fixe a antissimetria $\delta=\phi$. Temos $\Gamma_{+}=\mathbf{S}$. Considerando os geradores obtidos no Exemplo 2.2.3 e em (5.13), segue do Lema 2.2.1 que

$$
u_{1}(x, z)=x_{1}, u_{2}(x, z)=\left|z_{1}\right|^{2}, \ldots, u_{n+1}(x, z)=\left|z_{n}\right|^{2}
$$

é uma base de Hilbert para o anel $\mathcal{P}_{\mathbb{R}^{2} \times \mathbb{C}^{n}}\left(\Gamma_{+}\right)$, e do Lema 2.2 .2 que

$$
\begin{gathered}
\left(x_{1}, x_{2}, 0, \ldots, 0\right),(0,1,0, \ldots, 0),\left(0,0, z_{1}, \ldots, 0\right),\left(0,0, i z_{1}, \ldots, 0\right), \ldots, \\
\left(0,0,0, \ldots, z_{n}\right),\left(0,0,0, \ldots, i z_{n}\right)
\end{gathered}
$$

são geradores para $\overrightarrow{\mathcal{P}}_{\mathbb{R}^{2} \times \mathbb{C}^{n}}\left(\Gamma_{+}\right)$.

2. Agora, aplicamos o Algoritmo 1.4.1 para obter geradores para $\overrightarrow{\mathcal{Q}}_{\mathbb{R}^{2} \times \mathbb{C}^{n}}(\Gamma)$ sobre $\mathcal{P} \mathbb{R}^{2} \times \mathbb{C}^{n}(\Gamma)$ :

$H_{0}(x, z)=(0,1,0, \ldots, 0)$,

$H_{1}(x, z)=\left(0,0, i z_{1}, \ldots, 0\right)$,

$\vdots$

$H_{n}(x, z)=\left(0,0,0, \ldots, i z_{n}\right)$.

Usando os operadores de Reynolds $R$ e $S$, segue do Teorema 1.4 .2 que $\mathcal{P}_{\mathbb{R}^{2} \times \mathbb{C}^{n}}(\Gamma)$ tem a mesma base de Hilbert do anel $\mathcal{P}_{\mathbb{R}^{2} \times \mathbb{C}^{n}}\left(\Gamma_{+}\right)$, uma vez que, $R\left(u_{i}\right)=u_{i}$ e $S\left(u_{i}\right)=0$ para $1 \leq i \leq n$. 
3. Tome $\tilde{\Gamma}=\mathbf{S} \rtimes\left(\mathbf{Z}_{2}^{\phi} \times \mathbf{Z}_{2}^{\psi}\right)$ e fixe a antissimetria $\delta=\psi$. Considere a representação dual de $\mathbf{Z}_{2}^{\phi}$ em $\mathbb{R}^{2} \times \mathbb{C}^{n}$. Então, $\tilde{\Gamma}_{+}=\mathbf{S} \rtimes \mathbf{Z}_{2}^{\phi}$.

Considere os geradores para $\overrightarrow{\mathcal{P}}_{\mathbb{R}^{2} \times \mathbb{C}^{n}}\left(\tilde{\Gamma}_{+}\right)$e $\mathcal{P}_{\mathbb{R}^{2} \times \mathbb{C}^{n}}\left(\tilde{\Gamma}_{+}\right)$obtidos no passo 2 e aplique o Algoritmo 1.4.1.

- Tome $\tilde{u}_{0}(x, z)=1$. Temos, $\tilde{u}_{1}(x, z)=\left(\frac{1-a_{0}}{2}\right) u_{1} \quad$ e $\quad \tilde{u}_{i+1}(x, z)=0$ para $1 \leq i \leq n$.

- Faça $H_{0 j}(x, z)=H_{j}(x, z)$ e $H_{1 j}(x, z)=\left(\frac{1-a_{0}}{2}\right) u_{1}(x, z) H_{j}(x, z)$ para $0 \leq j \leq n$.

- Faça $\tilde{H}_{0 j}(x, z)$ e $\tilde{H}_{1 j}(x, z)$ para $0 \leq j \leq n$ :

$$
\begin{aligned}
& \tilde{H}_{00}(x, z)=\left(\frac{1+a_{0}}{2}\right) H_{0}(x, z), \\
& \tilde{H}_{0 l}(x, z)=H_{l}(x, z), \\
& \tilde{H}_{10}(x, z)=\left(\frac{1-a_{0}}{2}\right) u_{1}(x, z) H_{0}(x, z), \\
& \tilde{H}_{1 l}(x, z)=0 \text { para } 1 \leq l \leq n .
\end{aligned}
$$

Estes são os geradores para $\overrightarrow{\mathcal{Q}}_{\mathbb{R}^{2} \times \mathbb{C}^{n}}(\tilde{\Gamma})$ sobre $\mathcal{P}_{\mathbb{R}^{2} \times \mathbb{C}^{n}}(\tilde{\Gamma})$.

4. Para determinar a base de Hilbert para $\mathcal{P}_{\mathbb{R}^{2} \times \mathbb{C}^{n}}(\tilde{\Gamma})$ considere a base de Hilbert para

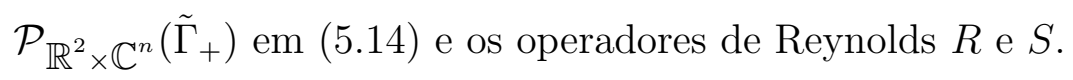

Como $R\left(u_{1}\right)=\left(\frac{1+a_{0}}{2}\right) u_{1}, R\left(u_{i+1}\right)=u_{i+1}, S\left(u_{1}\right)=\tilde{u}_{1}$ e $S\left(u_{i+1}\right)=0$, segue do Teorema 1.4 .2 que

$$
\tilde{u}_{1}^{2}(x, z)=\left(\frac{1-a_{0}}{2}\right) u_{1}^{2}(x, z), \quad\left(\frac{1+a_{0}}{2}\right) u_{1}(x, z), \quad u_{l+1}(x, z), \quad 1 \leq i \leq n,
$$

formam uma base de Hilbert para $\mathcal{P}_{\mathbb{R}^{2} \times \mathbb{C}^{n}}(\tilde{\Gamma})$.

Note que todos os geradores dependem apenas de $a_{0}$. Isso acontece porque cada $u_{i+1}$, $1 \leq i \leq n$, é invariante na coordenada $z$ pela ação de $\delta=\psi$. Como $a_{0}^{2}=1$ há dois casos para considerar: 
Se $a_{0}=-1$, então a forma normal $\mathbf{Z}_{2}^{\phi} \times \mathbf{Z}_{2}^{\psi}$-reversível-equivariante em coordenadas complexas é dada por

$$
\begin{aligned}
& \dot{x}_{1}=x_{2} \\
& \dot{x}_{2}=x_{1} f_{0}\left(x_{1}^{2},\left|z_{1}\right|^{2}, \ldots,\left|z_{n}\right|^{n}\right) \\
& \dot{z}_{1}=-i \omega_{1} z_{1}+i z_{1} f_{1}\left(x_{1}^{2},\left|z_{1}\right|^{2}, \ldots,\left|z_{n}\right|^{n}\right) \\
& \dot{z}_{2}=-i \omega_{2} z_{2}+i z_{2} f_{2}\left(x_{1}^{2},\left|z_{1}\right|^{2}, \ldots,\left|z_{n}\right|^{n}\right) \\
& \vdots \\
& \dot{z}_{n}=-i \omega_{2} z_{n}+i z_{n} f_{n}\left(x_{1}^{2},\left|z_{1}\right|^{2}, \ldots,\left|z_{n}\right|^{n}\right),
\end{aligned}
$$

$\operatorname{para} f_{i}: \mathbb{R}^{n+1}, 0 \rightarrow \mathbb{R}, 0 \leq i \leq n$.

Se $a_{0}=1$, então $\phi=\psi$ e, neste caso, obtemos a forma normal $\mathbf{Z}_{2}^{\phi}$-reversívelequivariante em coordenadas complexas:

$$
\begin{aligned}
& \dot{x}_{1}=x_{2} \\
& \dot{x}_{2}=f_{0}\left(x_{1},\left|z_{1}\right|^{2}, \ldots,\left|z_{n}\right|^{n}\right) \\
& \dot{z}_{1}=-i \omega_{1} z_{1}+i z_{1} f_{1}\left(x_{1},\left|z_{1}\right|^{2}, \ldots,\left|z_{n}\right|^{n}\right) \\
& \dot{z}_{2}=-i \omega_{2} z_{2}+i z_{2} f_{2}\left(x_{1},\left|z_{1}\right|^{2}, \ldots,\left|z_{n}\right|^{n}\right) \\
& \vdots \\
& \dot{z}_{n}=-i \omega_{2} z_{n}+i z_{n} f_{n}\left(x_{1},\left|z_{1}\right|^{2}, \ldots,\left|z_{n}\right|^{n}\right),
\end{aligned}
$$

para $f_{i}: \mathbb{R}^{n+1}, 0 \rightarrow \mathbb{R}, 0 \leq i \leq n$.

Observação 5.3.2. A forma normal (5.16) foi obtida por Lima e Teixeira em [44]. Os autores usam o método clássico desenvolvido por Belitskii sem simetrias para obter uma pré forma normal e impõem a reversibilidade de $\boldsymbol{Z}_{2}^{\phi}$ a posteriori. Enfatizamos a importância deste exemplo em nosso estudo já que foi o motivador para a obtenção do Teorema 3.3.1, resultado geral para grupos de Lie compactos.

\subsubsection{Ressonância do tipo $\left(n_{1}: n_{2}\right)$ em $\mathbb{R}^{2} \times \mathbb{C}^{2}$}

Nesta subseção calculamos as formas normais $\mathbf{Z}_{2}^{\phi} \times \mathbf{Z}_{2}^{\psi}$-reversíveis-equivariantes de (5.1) para $n=2$ quando $n_{1} \omega_{2}-n_{2} \omega_{1}=0, n_{1}, n_{2} \in \mathbb{N}$. Sob estas condições, o sistema (5.1) é chamado de $\left(n_{1}: n_{2}\right)$-ressonante. Observamos aqui que a dedução da forma normal via 
o cálculo do ker $A d_{L^{t}}^{k}$ se torna complicada à medida que os valores de $n_{1}$ e $n_{2}$ se tornam maiores. De fato, isto é visto em [44] e [50], onde os autores lidam com alguns valores particulares de $n_{1}$ e $n_{2}$. Com o Teorema 3.3.5 contornamos este problema e encontramos formas normais para quaisquer valores de $n_{1}$ e $n_{2}$.

Pelo Teorema 5.3.1, neste caso temos $\mathbf{S}=\mathbf{R} \times \mathbf{T}$. Aqui, usamos coordenadas $(x, z)=$ $\left(x_{1}, x_{2}, z_{1}, z_{2}\right) \in \mathbb{R}^{2} \times \mathbb{C}^{2}$, nas quais a ação de $\mathbf{R}$ em $\mathbb{R}^{2}$ é dada em (5.10) e a ação de $\mathbf{T}$ em $\mathbb{C}^{2}$ é da forma:

$$
\theta\left(z_{1}, z_{2}\right)=\left(e^{i n_{1} \theta} z_{1}, e^{i n_{2} \theta} z_{2}\right)
$$

Invariantes e equivariantes segundo a ação de T em (5.17) são dados em [35, Teorema 4.2]:

$$
\left|z_{1}\right|^{2},\left|z_{2}\right|^{2}, \operatorname{Re}\left(z_{1}^{n_{2}} \bar{z}_{2}^{n_{1}}\right), \operatorname{Im}\left(z_{1}^{n_{2}} \bar{z}_{2}^{n_{1}}\right)
$$

e,

$$
\begin{gathered}
\left(z_{1}, 0\right),\left(z_{1} i, 0\right),\left(\bar{z}_{1}^{n_{2}-1} z_{2}^{n_{1}}, 0\right),\left(\bar{z}_{1}^{n_{2}-1} z_{2}^{n_{1}} i, 0\right),\left(0, z_{2}\right),\left(0, z_{2} i\right) \\
\left(0, z_{1}^{n_{2}} \bar{z}_{2}^{n_{1}-1}\right),\left(0, z_{1}^{n_{2}} \bar{z}_{2}^{n_{1}-1} i\right)
\end{gathered}
$$

respectivamente.

As ações de $\phi$ e $\psi$ em $\mathbb{R}^{2} \times \mathbb{C}^{2}$ são dadas em (5.11) e (5.12) para $n=2$.

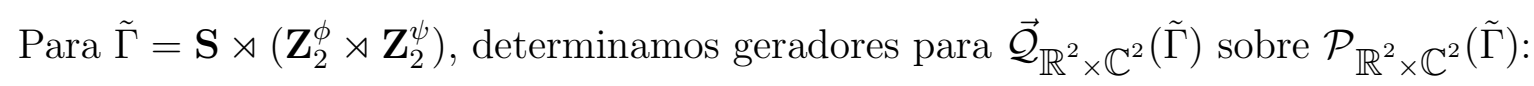

1. Seja $\Gamma=\mathbf{S} \rtimes \mathbf{Z}_{2}^{\phi}$ e fixe a antissimetria $\delta=\phi$. Temos $\Gamma_{+}=\mathbf{S}$. Do Lema 2.2.1 segue que

$$
\begin{gathered}
u_{1}(x, z)=x_{1}, \quad u_{2}(x, z)=\left|z_{1}\right|^{2}, \quad u_{3}(x, z)=\left|z_{2}\right|^{2}, \\
u_{4}(x, z)=\operatorname{Re}\left(z_{1}^{n_{2}} \bar{z}_{2}^{n_{1}}\right) \quad \text { e } \quad u_{5}(x, z)=\operatorname{Im}\left(z_{1}^{n_{2}} \bar{z}_{2}^{n_{1}}\right)
\end{gathered}
$$

é uma base de Hilbert para $\mathcal{P}_{\mathbb{R}^{2} \times \mathbb{C}^{2}}\left(\Gamma_{+}\right)$. Do Lema 2.2 .2 segue que os geradores para $\overrightarrow{\mathcal{P}}_{\mathbb{R}^{2} \times \mathbb{C}^{2}}\left(\Gamma_{+}\right)$são dados por:

$$
\begin{gathered}
\left(x_{1}, x_{2}, 0,0\right),(0,1,0,0),\left(0,0, z_{1}, 0\right),\left(0,0, i z_{1}, 0\right),\left(0,0, \bar{z}_{1}^{n_{2}-1} z_{2}^{n_{1}}, 0\right), \\
\left(0,0, i \bar{z}_{1}^{n_{2}-1} z_{2}^{n_{1}}, 0\right),\left(0,0,0, z_{2}\right),\left(0,0,0, i z_{2}\right),\left(0,0,0, z_{1}^{n_{2}} \bar{z}_{2}^{n_{1}-1}\right),\left(0,0,0, i z_{1}^{n_{2}} \bar{z}_{2}^{n_{1}-1}\right) .
\end{gathered}
$$

2. Agora, aplicamos o Algoritmo 1.4 .1 e obtemos geradores para $\overrightarrow{\mathcal{Q}}_{\mathbb{R}^{2} \times \mathbb{C}^{2}}(\Gamma)$ sobre $\mathcal{P}_{\mathbb{R}^{2} \times \mathbb{C}^{2}}(\Gamma)$ : 


$$
\begin{aligned}
& H_{0}(x, z)=(0,1,0,0), \\
& H_{1}(x, z)=\left(x_{1} \operatorname{Im}\left(z_{1}^{n_{2}} \bar{z}_{2}^{n_{1}}\right), x_{2} \operatorname{Im}\left(z_{1}^{n_{2}} \bar{z}_{2}^{n_{1}}\right), 0,0\right), \\
& H_{2}(x, z)=\left(0,0, i z_{1}, 0\right), \\
& H_{3}(x, z)=\left(0,0, i \bar{z}_{1}^{n_{2}-1} z_{2}^{n_{1}}, 0\right), \\
& H_{4}(x, z)=\left(0,0, z_{1} \operatorname{Im}\left(z_{1}^{n_{2}} \bar{z}_{2}^{n_{1}}\right), 0\right), \\
& H_{5}(x, z)=\left(0,0, \bar{z}_{1}^{n_{2}-1} z_{2}^{n_{1}} \operatorname{Im}\left(z_{1}^{n_{2}} \bar{z}_{2}^{n_{1}}\right), 0\right), \\
& H_{6}(x, z)=\left(0,0,0, i z_{2}\right), \\
& H_{7}(x, z)=\left(0,0,0, i z_{1}^{n_{2}} \bar{z}_{2}^{n_{1}-1}\right), \\
& H_{8}(x, z)=\left(0,0,0, z_{2} \operatorname{Im}\left(z_{1}^{n_{2}} \bar{z}_{2}^{n_{1}}\right)\right), \\
& H_{9}(x, z)=\left(0,0,0, z_{1}^{n_{2}} \bar{z}_{2}^{n_{1}-1} \operatorname{Im}\left(z_{1}^{n_{2}} \bar{z}_{2}^{n_{1}}\right)\right) .
\end{aligned}
$$

Usando os operadores de Reynolds $R, S$ e o Teorema 1.4.2, obtemos

$$
u_{1}, \quad u_{2}, u_{3}, \quad u_{4}
$$

dados em 5.20), como base de Hilbert para $\mathcal{P}_{\mathbb{R}^{2} \times \mathbb{C}^{2}}(\Gamma)$.

De fato, $R\left(u_{i}\right)=u_{i}, R\left(u_{5}\right)=0, S\left(u_{i}\right)=0, S\left(u_{5}\right)=u_{5}$ para $1 \leq i \leq 4$. Note que $u_{5}^{2}=u_{2}^{n_{2}} u_{3}^{n_{1}}-u_{4}^{2}$. Assim, o resultado segue.

3. Seja $\tilde{\Gamma}=\mathbf{S} \rtimes\left(\mathbf{Z}_{2}^{\phi} \times \mathbf{Z}_{2}^{\psi}\right)$ e fixe a antissimetria $\delta=\psi$. Considere a representação dual de $\mathbf{Z}_{2}^{\phi}$ em $\mathbb{R}^{2} \times \mathbb{C}^{2}$. Então, $\tilde{\Gamma}_{+}=\mathbf{S} \rtimes \mathbf{Z}_{2}^{\phi}$. Considere os geradores para $\mathcal{P}_{\mathbb{R}^{2} \times \mathbb{C}^{2}\left(\tilde{\Gamma}_{+}\right)}$ e $\overrightarrow{\mathcal{P}}_{\mathbb{R}^{2} \times \mathbb{C}^{2}}\left(\tilde{\Gamma}_{+}\right)$calculados no item 2 e aplique o Algoritmo 1.4 .1 novamente:

- Faça $\tilde{u}_{0}(x, z)=1$. Temos,

$$
\begin{aligned}
& \tilde{u}_{1}(x, z)=\left(\frac{1-a_{0}}{2}\right) u_{1}(x, z), \quad \tilde{u}_{2}(x, z)=\tilde{u}_{3}(x, z)=0 \quad \mathrm{e} \\
& \tilde{u}_{4}(x, z)=\left(\frac{1-a_{1}^{n_{2}} a_{2}^{n_{1}}}{2}\right) u_{4}(x, z) .
\end{aligned}
$$

- Faça

$$
\begin{aligned}
& H_{0 j}(x, z)=H_{j}(x, z), H_{1 j}(x, z)=\left(\frac{1-a_{0}}{2}\right) u_{1}(x, z) H_{j}(x, z) \mathrm{e} \\
& H_{4 j}(x, z)=\left(\frac{1-a_{1}^{n_{2}} a_{2}^{n_{1}}}{2}\right) u_{4}(x, z) H_{j}(x, z) \text { para } 0 \leq j \leq 9 .
\end{aligned}
$$


- Calcule $\tilde{H}_{0 j}(x, z), \tilde{H}_{1 j}(x, z)$ e $\tilde{H}_{4 j}(x, z)$ para $0 \leq j \leq 9$ :

$$
\begin{aligned}
& \tilde{H}_{00}(x, z)=\left(\frac{1+a_{0}}{2}\right) H_{0}(x, z), \\
& \tilde{H}_{0 l}(x, z)=\left(\frac{1+a_{1}^{n_{2}} a_{2}^{n_{1}}}{2}\right) H_{l}(x, z), \\
& \tilde{H}_{0 k}=H_{k}(x, z), \\
& \tilde{H}_{10}(x, z)=\left(\frac{1-a_{0}}{2}\right) u_{1}(x, z) H_{0}(x, z), \\
& \tilde{H}_{1 l}(x, z)=\left(\frac{1+a_{0} a_{1}^{n_{2}} a_{2}^{n_{1}}}{2}\right)\left(\frac{1-a_{0}}{2}\right) u_{1}(x, z) H_{l}(x, z), \\
& \tilde{H}_{1 k}(x, z)=0, \\
& \tilde{H}_{40}(x, z)=\left(\frac{1-a_{0}}{2}\right)\left(\frac{1-a_{1}^{n_{2}} a_{2}^{n_{1}}}{2}\right) u_{4}(x, z) H_{0}(x, z), \\
& \tilde{H}_{4 l}(x, z)=\left(\frac{1-a_{1}^{n_{2}} a_{2}^{n_{1}}}{2}\right) u_{4}(x, z) H_{l}(x, z) \\
& \tilde{H}_{4 k}(x, z)=0, \text { para } l=1,3,4,7,8 \text { e } k=2,5,6,9 .
\end{aligned}
$$

Estes são os geradores para $\overrightarrow{\mathcal{Q}}_{\mathbb{R}^{2} \times \mathbb{C}^{2}}(\tilde{\Gamma})$ sobre $\mathcal{P}_{\mathbb{R}^{2} \times \mathbb{C}^{2}}(\tilde{\Gamma})$.

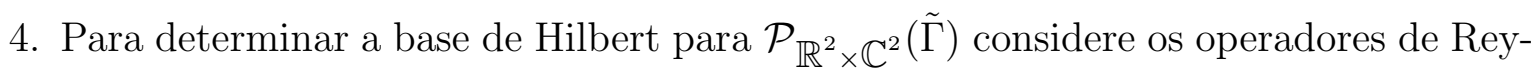
nolds $R, S$ e a base de Hilbert para $\mathcal{P}_{\mathbb{R}^{2} \times \mathbb{C}^{2}}\left(\tilde{\Gamma}_{+}\right)$em (5.21).

Como $R\left(u_{1}\right)=\left(\frac{1+a_{0}}{2}\right) u_{1}, \quad R\left(u_{2}\right)=u_{2}, \quad R\left(u_{3}\right)=u_{3}, \quad R\left(u_{4}\right)=\left(\frac{1+a_{1}^{n_{2}} a_{2}^{n_{1}}}{2}\right) u_{4}$, $S\left(u_{1}\right)=\tilde{u}_{1}, \quad S\left(u_{2}\right)=S\left(u_{3}\right)=0$ e $S\left(u_{4}\right)=\tilde{u}_{4}$, segue do Teorema 1.4 .2 que

$$
\left(\frac{1+a_{0}}{2}\right) u_{1}, u_{2}, u_{3},\left(\frac{1+a_{1}^{n_{2}} a_{2}^{n_{1}}}{2}\right) u_{4}, \tilde{u}_{1}^{2}, \tilde{u}_{4}^{2} \text { e } \tilde{u}_{1} \tilde{u}_{4}
$$

formam uma base de Hilbert para $\mathcal{P}_{\mathbb{R}^{2} \times \mathbb{C}^{2}}(\tilde{\Gamma})$.

Para $a_{i}= \pm 1, \quad i=1,2,3$, consideramos todas as possibilidades e encontramos 4 tipos de formas normais. Os tipos estão descritos na Tabela 5.1 com os respectivos geradores na Tabela 5.2. Observe na Tabela 5.1 que o Tipo $A$ recai no caso $\mathbf{Z}_{2}^{\phi}$-reversível-equivariante (puramente reversível, sem simetrias não triviais).

Resumimos o que aparece acima: 


\begin{tabular}{|c|c|c|c|}
\hline \multirow[t]{7}{*}{$a_{0}=1$} & $a_{1}=a_{2}=1$ & - & Tipo A \\
\hline & \multirow{2}{*}{$a_{1}=a_{2}=-1$} & $n_{1}+n_{2}$ par & Tipo A \\
\hline & & $n_{1}+n_{2}$ ímpar & Tipo B \\
\hline & \multirow[t]{2}{*}{$a_{1}=-a_{2}=1$} & $n_{1}$ par & Tipo A \\
\hline & & $n_{1}$ ímpar & Tipo B \\
\hline & \multirow[t]{2}{*}{$a_{1}=-a_{2}=-1$} & $n_{2}$ par & Tipo A \\
\hline & & $n_{2}$ ímpar & Tipo B \\
\hline \multirow[t]{7}{*}{$a_{0}=-1$} & $a_{1}=a_{2}=1$ & - & Tipo C \\
\hline & \multirow[t]{2}{*}{$a_{1}=a_{2}=-1$} & $n_{1}+n_{2}$ par & Tipo C \\
\hline & & $n_{1}+n_{2}$ ímpar & Tipo D \\
\hline & \multirow[t]{2}{*}{$a_{1}=-a_{2}=1$} & $n_{1}$ par & Tipo C \\
\hline & & $n_{1}$ ímpar & Tipo D \\
\hline & \multirow[t]{2}{*}{$a_{1}=-a_{2}=-1$} & $n_{2}$ par & Tipo C \\
\hline & & $n_{2}$ ímpar & Tipo D \\
\hline
\end{tabular}

Tabela 5.1: Formas normais de campos $\mathbf{Z}_{2} \times \mathbf{Z}_{2}$-reversíveis-equivariantes

\begin{tabular}{|c|c|c|}
\hline & $\overrightarrow{\mathcal{Q}}_{\mathbb{R}^{2} \times \mathbb{C}^{2}}(\tilde{\Gamma})$ & $\mathcal{P}_{\mathbb{R}^{2} \times \mathbb{C}^{2}(\tilde{\Gamma})}$ \\
\hline Tipo A & $H_{j}, 0 \leq j \leq 9$ & $u_{1}, u_{2}, u_{3}, u_{4}$ \\
\hline Tipo B & $H_{k}, u_{4} H_{l}$ para $k=0,2,5,6,9$ e $l=1,3,4,7,8$ & $u_{1}, u_{2}, u_{3}, u_{4}^{2}$ \\
\hline Tipo C & $u_{1} H_{0}, H_{k}$ para $1 \leq k \leq 9$ & $u_{1}^{2}, u_{2}, u_{3}, u_{4}$ \\
\hline Tipo D & $H_{k}, u_{1} H_{l}, u_{4} H_{l}$ & $u_{1}^{2}, u_{2}, u_{3}, u_{4}^{2}, u_{1} u_{4}$ \\
& para $k=2,5,6,9$ e $l=0,1,3,4,7,8$ & $u_{1}^{2}, u_{2}, u_{3}, u_{4}^{2}, u_{1} u_{4}$ \\
\hline
\end{tabular}

Tabela 5.2: Geradores em $\mathbb{R}^{2} \times \mathbb{C}^{2}$

Teorema 5.3.3. Seja $\dot{x}=h(x)$ um sistema $\mathbf{Z}_{2}^{\phi} \times \mathbf{Z}_{2}^{\psi}$-reversivel-equivariante, com $L=$ $(d h)_{0}$ definida em (5.2) para $n=2$ e $n_{1} \omega_{2}-n_{2} \omega_{1}=0$. Então, este sistema é formalmente conjugado a um dos sistemas abaixo:

Tipo A:

$$
\begin{aligned}
& \dot{x}_{1}=x_{2}+x_{1} \operatorname{Im}\left(z_{1}^{n_{2}} \bar{z}_{2}^{n_{1}}\right) f_{0}(X) \\
& \dot{x}_{2}=f_{1}(X)+x_{2} \operatorname{Im}\left(z_{1}^{n_{2}} \bar{z}_{2}^{n_{1}}\right) f_{0}(X) \\
& \dot{z}_{1}=-i \omega_{1} z_{1}+i z_{1} f_{2}(X)+i \bar{z}_{1}^{n_{2}-1} z_{2}^{n_{2}} f_{3}(X)+z_{1} \operatorname{Im}\left(z_{1}^{n_{2}} \bar{z}_{2}^{n_{1}}\right) f_{4}(X)+\bar{z}_{1}^{n_{2}-1} z_{2}^{n_{2}} \operatorname{Im}\left(z_{1}^{n_{2}} \bar{z}_{2}^{n_{1}}\right) f_{5}(X) \\
& \dot{z}_{2}=-i \omega_{2} z_{2}+i z_{2} f_{6}(X)+i z_{1}^{n_{2}} \bar{z}_{2}^{n_{1}-1} f_{7}(X)+z_{2} \operatorname{Im}\left(z_{1}^{n_{2}} \bar{z}_{2}^{n_{1}}\right) f_{8}(X)+z_{1}^{n_{2}} \bar{z}_{2}^{n_{1}-1} \operatorname{Im}\left(z_{1}^{n_{2}} \bar{z}_{2}^{n_{1}}\right) f_{9}(X)
\end{aligned}
$$


para $f_{i}: \mathbb{R}^{4}, 0 \rightarrow \mathbb{R}, 0 \leq i \leq 9, X=\left(x_{1},\left|z_{1}\right|^{2},\left|z_{2}\right|^{2}, \operatorname{Re}\left(z_{1}^{n_{2}} \bar{z}_{2}^{n_{1}}\right)\right)$.

Tipo B:

$$
\begin{aligned}
\dot{x}_{1}= & x_{2}+x_{1} \operatorname{Re}\left(z_{1}^{n_{2}} \bar{z}_{2}^{n_{1}}\right) \operatorname{Im}\left(z_{1}^{n_{2}} \bar{z}_{2}^{n_{1}}\right) f_{0}(X) \\
\dot{x}_{2}= & f_{1}(X)+x_{2} \operatorname{Re}\left(z_{1}^{n_{2}} \bar{z}_{2}^{n_{1}}\right) \operatorname{Im}\left(z_{1}^{n_{2}} \bar{z}_{2}^{n_{1}}\right) f_{0}(X) \\
\dot{z}_{1}= & -i \omega_{1} z_{1}+i z_{1} f_{2}(X)+\bar{z}_{1}^{n_{2}-1} z_{2}^{n_{2}} \operatorname{Im}\left(z_{1}^{n_{2}} \bar{z}_{2}^{n_{1}}\right) f_{3}(X)+i \bar{z}_{1}^{n_{2}-1} z_{2}^{n_{2}} \operatorname{Re}\left(z_{1}^{n_{2}} \bar{z}_{2}^{n_{1}}\right) f_{4}(X)+ \\
& +z_{1} \operatorname{Re}\left(z_{1}^{n_{2}} \bar{z}_{2}^{n_{1}}\right) \operatorname{Im}\left(z_{1}^{n_{2}} \bar{z}_{2}^{n_{1}}\right) f_{5}(X) \\
\dot{z}_{2}= & -i \omega_{2} z_{2}+i z_{2} f_{6}(X)+z_{1}^{n_{2}} \bar{z}_{2}^{n_{1}-1} \operatorname{Im}\left(z_{1}^{n_{2}} \bar{z}_{2}^{n_{1}}\right) f_{7}(X)+i z_{1}^{n_{2}} \bar{z}_{2}^{n_{1}-1} \operatorname{Re}\left(z_{1}^{n_{2}} \bar{z}_{2}^{n_{1}}\right) f_{8}(X)+ \\
& +z_{2} \operatorname{Re}\left(z_{1}^{n_{2}} \bar{z}_{2}^{n_{1}}\right) \operatorname{Im}\left(z_{1}^{n_{2}} \bar{z}_{2}^{n_{1}}\right) f_{9}(X)
\end{aligned}
$$

para $f_{i}: \mathbb{R}^{4}, 0 \rightarrow \mathbb{R}, 0 \leq i \leq 9, X=\left(x_{1},\left|z_{1}\right|^{2},\left|z_{2}\right|^{2}, \operatorname{Re}^{2}\left(z_{1}^{n_{2}} \bar{z}_{2}^{n_{1}}\right)\right)$

Tipo C:

$$
\begin{aligned}
& \dot{x}_{1}=x_{2}+x_{1} \operatorname{Im}\left(z_{1}^{n_{2}} \bar{z}_{2}^{n_{1}}\right) f_{0}(X) \\
& \dot{x}_{2}=x_{1} f_{1}(X)+x_{2} \operatorname{Im}\left(z_{1}^{n_{2}} \bar{z}_{2}^{n_{1}}\right) f_{0}(X) \\
& \dot{z}_{1}=-i \omega_{1} z_{1}+i z_{1} f_{2}(X)+i \bar{z}_{1}^{n_{2}-1} z_{2}^{n_{2}} f_{3}(X)+z_{1} \operatorname{Im}\left(z_{1}^{n_{2}} \bar{z}_{2}^{n_{1}}\right) f_{4}(X)+\bar{z}_{1}^{n_{2}-1} z_{2}^{n_{2}} \operatorname{Im}\left(z_{1}^{n_{2}} \bar{z}_{2}^{n_{1}}\right) f_{5}(X) \\
& \dot{z}_{2}=-i \omega_{2} z_{2}+i z_{2} f_{6}(X)+i z_{1}^{n_{2}} \bar{z}_{2}^{n_{1}-1} f_{7}(X)+z_{2} \operatorname{Im}\left(z_{1}^{n_{2}} \bar{z}_{2}^{n_{1}}\right) f_{8}(X)+z_{1}^{n_{2}} \bar{z}_{2}^{n_{1}-1} \operatorname{Im}\left(z_{1}^{n_{2}} \bar{z}_{2}^{n_{1}}\right) f_{9}(X) \\
& \text { para } f_{i}: \mathbb{R}^{4}, 0 \rightarrow \mathbb{R}, 0 \leq i \leq 9, X=\left(x_{1}^{2},\left|z_{1}\right|^{2},\left|z_{2}\right|^{2}, \operatorname{Re}\left(z_{1}^{n_{2}} \bar{z}_{2}^{n_{1}}\right)\right) .
\end{aligned}
$$

\section{Tipo D:}

$$
\begin{aligned}
\dot{x}_{1}= & x_{2}+x_{1}^{2} \operatorname{Im}\left(z_{1}^{n_{2}} \bar{z}_{2}^{n_{1}}\right) f_{0}(X)+x_{1} \operatorname{Re}\left(z_{1}^{n_{2}} \bar{z}_{2}^{n_{1}}\right) \operatorname{Im}\left(z_{1}^{n_{2}} \bar{z}_{2}^{n_{1}}\right) f_{1}(X) \\
\dot{x}_{2}= & \left.x_{1} f_{2}(X)+x_{1} x_{2} \operatorname{Im}\left(z_{1}^{n_{2}} \bar{z}_{2}^{n_{1}}\right) f_{0}(X)+\operatorname{Re}\left(z_{1}^{n_{2}} \bar{z}_{2}^{n_{1}}\right)\right) f_{3}(X)+x_{2} \operatorname{Re}\left(z_{1}^{n_{2}} \bar{z}_{2}^{n_{1}}\right) \operatorname{Im}\left(z_{1}^{n_{2}} \bar{z}_{2}^{n_{1}}\right) f_{1}(X) \\
\dot{z}_{1}= & -i \omega_{1} z_{1}+i z_{1} f_{4}(X)+\bar{z}_{1}^{n_{2}-1} z_{2}^{n_{2}} \operatorname{Im}\left(z_{1}^{n_{2}} \bar{z}_{2}^{n_{1}}\right) f_{5}(X)+i x_{1} \bar{z}_{1}^{n_{2}-1} z_{2}^{n_{2}} f_{6}(X)+ \\
& +x_{1} z_{1} \operatorname{Im}\left(z_{1}^{n_{2}} \bar{z}_{2}^{n_{1}}\right) f_{7}(X)+i \bar{z}_{1}^{n_{2}-1} z_{2}^{n_{2}} \operatorname{Re}\left(z_{1}^{n_{2}} \bar{z}_{2}^{n_{1}}\right) f_{8}(X)+z_{1} \operatorname{Re}\left(z_{1}^{n_{2}} \bar{z}_{2}^{n_{1}}\right) \operatorname{Im}\left(z_{1}^{n_{2}} \bar{z}_{2}^{n_{1}}\right) f_{9}(X)+ \\
\dot{z}_{2}= & -i \omega_{2} z_{2}+i z_{2} f_{10}(X)+z_{1}^{n_{2}} \bar{z}_{2}^{n_{1}-1} \operatorname{Im}\left(z_{1}^{n_{2}} \bar{z}_{2}^{n_{1}}\right) f_{11}(X)+i x_{1} z_{1}^{n_{2}} \bar{z}_{2}^{n_{1}-1} f_{12}(X)+ \\
& +x_{1} z_{2} \operatorname{Im}\left(z_{1}^{n_{2}} \bar{z}_{2}^{n_{1}}\right) f_{13}(X)+i z_{1}^{n_{2}} \bar{z}_{2}^{n_{1}-1} \operatorname{Re}\left(z_{1}^{n_{2}} \bar{z}_{2}^{n_{1}}\right) f_{14}(X)+z_{2} \operatorname{Re}\left(z_{1}^{n_{2}} \bar{z}_{2}^{n_{1}}\right) \operatorname{Im}\left(z_{1}^{n_{2}} \bar{z}_{2}^{n_{1}}\right) f_{15}(X)
\end{aligned}
$$

para $f_{i}: \mathbb{R}^{4}, 0 \rightarrow \mathbb{R}, 0 \leq i \leq 15, X=\left(x_{1}^{2},\left|z_{1}\right|^{2},\left|z_{2}\right|^{2}, \operatorname{Re}^{2}\left(z_{1}^{n_{2}} \bar{z}_{2}^{n_{1}}\right), x_{1} \operatorname{Re}\left(z_{1}^{n_{2}} \bar{z}_{2}^{n_{1}}\right)\right)$ 


\subsubsection{Ressonância do tipo $\left(n_{1}: n_{2}: 0\right)$ em $\mathbb{R}^{2} \times \mathbb{C}^{3}$}

Nesta subseção calculamos as formas normais $\mathbf{Z}_{2}^{\phi} \times \mathbf{Z}_{2}^{\psi}$-reversíveis-equivariantes de (5.1) para $n=3$. Aqui, supomos que $n_{1} \omega_{2}-n_{2} \omega_{1}=0, n_{1}, n_{2} \in \mathbb{N}$, e que $\omega_{3}$ é algebricamente independente com relação a $\omega_{1}$ e $\omega_{2}$. Neste caso, o sistema é chamado $\left(n_{1}: n_{2}: 0\right)$-ressonante. Enfatizamos novamente que através do Teorema 3.3 .5 determinamos formas normais para quaisquer valores de $n_{1}$ e $n_{2}$.

Pelo Teorema 5.3.1, $\mathbf{S}=\mathbf{R} \times \mathbf{T} \times \mathbf{T}$. Em coordenadas complexas $(x, z)=\left(x_{1}, x_{2}, z_{1}, z_{2}, z_{3}\right) \in$ $\mathbb{R}^{2} \times \mathbb{C}^{3}$ a ação de $\mathbf{S}$ é conhecida através da ação de $\mathbf{R}$ em $\mathbb{R}^{2}$ dada em $(5.10)$, e das ações de $\mathbf{T}$ em $\mathbb{C}_{2}$ e $\mathbf{T}$ em $\mathbb{C}$ :

$$
\theta\left(z_{1}, z_{2}\right)=\left(e^{i n_{1} \theta} z_{1}, e^{i n_{2} \theta} z_{2}\right) \quad \text { e } \theta z_{3}=e^{i \theta} z_{3}
$$

Considere ainda as ações de $\mathbf{Z}_{2}^{\phi}$ e $\mathbf{Z}_{2}^{\psi}$ em $\mathbb{R}^{2} \times \mathbb{C}^{3}$ dadas em (5.11) e (5.12), respectivamente, para $n=3$.

Para $\tilde{\Gamma}=\mathbf{S} \rtimes\left(\mathbf{Z}_{2}^{\phi} \times \mathbf{Z}_{2}^{\psi}\right)$, vamos determinar um conjunto de geradores para $\overrightarrow{\mathcal{Q}}_{\mathbb{R}^{2} \times \mathbb{C}^{3}}(\tilde{\Gamma})$

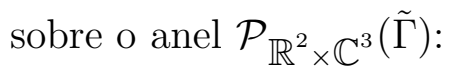

1. Seja $\Gamma=\mathbf{S} \rtimes \mathbf{Z}_{2}^{\phi}$ e fixe a antissimetria $\delta=\phi$. Temos $\Gamma_{+}=\mathbf{S}$. Considere os geradores dados no Exemplo 2.2 .3 e em (5.13), 5.18), 5.19.

Pelo Lema 2.2.1,

$$
\begin{gathered}
u_{1}(x, z)=x_{1}, \quad u_{2}(x, z)=\left|z_{1}\right|^{2}, \quad u_{3}(x, z)=\left|z_{2}\right|^{2}, \quad u_{4}(x, z)=\operatorname{Re}\left(z_{1}^{n_{2}} \bar{z}_{2}^{n_{1}}\right), \\
u_{5}(x, z)=\operatorname{Im}\left(z_{1}^{n_{2}} \bar{z}_{2}^{n_{1}}\right) \text { e } u_{6}(x, z)=\left|z_{3}\right|^{2}
\end{gathered}
$$

é uma base de Hilbert para $\mathcal{P}_{\mathbb{R}^{2} \times \mathbb{C}^{3}}\left(\Gamma_{+}\right)$.

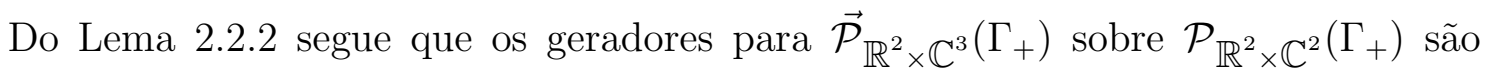
dados por:

$$
\begin{gathered}
\left(x_{1}, x_{2}, 0,0,0\right),(0,1,0,0,0),\left(0,0, z_{1}, 0,0\right),\left(0,0, i z_{1}, 0,0\right),\left(0,0, \bar{z}_{1}^{n_{2}-1} z_{2}^{n_{1}}, 0,0\right), \\
\left(0,0, i \bar{z}_{1}^{n_{2}-1} z_{2}^{n_{1}}, 0,0\right),\left(0,0,0, z_{2}, 0\right),\left(0,0,0, i z_{2}, 0\right),\left(0,0,0, z_{1}^{n_{2}} \bar{z}_{2}^{n_{1}-1}, 0\right) \\
\left(0,0,0, i z_{1}^{n_{2}} \bar{z}_{2}^{n_{1}-1}, 0\right),\left(0,0,0,0, z_{3}\right),\left(0,0,0,0, i z_{3}\right) .
\end{gathered}
$$


2. Agora, aplicamos o Algoritmo 1.4 .1 e obtemos geradores para $\overrightarrow{\mathcal{Q}}_{\mathbb{R}^{2} \times \mathbb{C}^{3}}(\Gamma)$ sobre $\mathcal{P}_{\mathbb{R}^{2} \times \mathbb{C}^{3}}(\Gamma)$ :

$$
\begin{aligned}
& H_{0}(x, z)=(0,1,0,0,0) \\
& H_{1}(x, z)=\left(x_{1} \operatorname{Im}\left(z_{1}^{n_{2}} \bar{z}_{2}^{n_{1}}\right), x_{2} \operatorname{Im}\left(z_{1}^{n_{2}} \bar{z}_{2}^{n_{1}}\right), 0,0,0\right) \\
& H_{2}(x, z)=\left(0,0, i z_{1}, 0,0\right) \\
& H_{3}(x, z)=\left(0,0, i \bar{z}_{1}^{n_{2}-1} z_{2}^{n_{1}}, 0,0\right) \\
& H_{4}(x, z)=\left(0,0, z_{1} \operatorname{Im}\left(z_{1}^{n_{2}} \bar{z}_{2}^{n_{1}}\right), 0,0\right) \\
& H_{5}(x, z)=\left(0,0, \bar{z}_{1}^{n_{2}-1} z_{2}^{n_{1}} \operatorname{Im}\left(z_{1}^{n_{2}} \bar{z}_{2}^{n_{1}}\right), 0,0\right), \\
& H_{6}(x, z)=\left(0,0,0, i z_{2}, 0\right) \\
& H_{7}(x, z)=\left(0,0,0, i z_{1}^{n_{2}} \bar{z}_{2}^{n_{1}-1}, 0\right) \\
& H_{8}(x, z)=\left(0,0,0, z_{2} \operatorname{Im}\left(z_{1}^{n_{2}} \bar{z}_{2}^{n_{1}}\right), 0\right) \\
& H_{9}(x, z)=\left(0,0,0, z_{1}^{n_{2}} \bar{z}_{2}^{n_{1}-1} \operatorname{Im}\left(z_{1}^{n_{2}} \bar{z}_{2}^{n_{1}}\right), 0\right), \\
& H_{10}(x, z)=\left(0,0,0,0, i z_{3}\right) \\
& H_{11}(x, z)=\left(0,0,0,0, z_{3} \operatorname{Im}\left(z_{1}^{n_{2}} \bar{z}_{2}^{n_{1}}\right)\right) .
\end{aligned}
$$

Usando os operadores de Reynolds $R, S$ e o Teorema 1.4.2, obtemos uma base de Hilbert para $\mathcal{P}_{\mathbb{R}^{2} \times \mathbb{C}^{2}}(\Gamma)$ :

$$
u_{1}, u_{2}, u_{3}, u_{4} \text { e } u_{6}
$$

De fato, já que $R\left(u_{i}\right)=u_{i}, R\left(u_{5}\right)=0, S\left(u_{i}\right)=0$ e $S\left(u_{5}\right)=u_{5}$ para $i=1,2,3,4,6$. Como $u_{5}^{2}=u_{2}^{n_{2}} u_{3}^{n_{1}}-u_{4}^{2}$, o resultado segue.

3. Seja $\tilde{\Gamma}=\mathbf{S} \rtimes\left(\mathbf{Z}_{2}^{\phi} \times \mathbf{Z}_{2}^{\psi}\right)$ e fixe a antissimetria $\delta=\psi$. Considere a representação

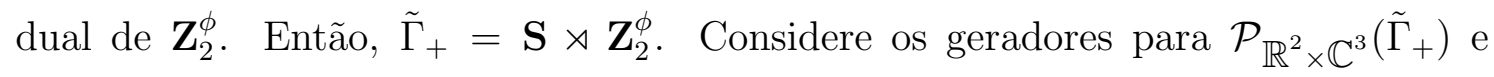
$\overrightarrow{\mathcal{P}}_{\mathbb{R}^{2} \times \mathbb{C}^{3}}\left(\tilde{\Gamma}_{+}\right)$calculados no item 2 e aplique o Algoritmo 1.4.1.

- Faça $\tilde{u}_{0}(x, z)=1$. Temos,

$$
\begin{aligned}
& \tilde{u}_{1}(x, z)=\left(\frac{1-a_{0}}{2}\right) u_{1}(x, z), \quad \tilde{u}_{2}(x, z)=\tilde{u}_{3}(x, z)=\tilde{u}_{6}(x, z)=0 \quad \mathrm{e} \\
& \tilde{u}_{4}(x, z)=\left(\frac{1-a_{1}^{n_{2}} a_{2}^{n_{1}}}{2}\right) u_{4}(x, z) .
\end{aligned}
$$


- Faça

$$
\begin{aligned}
& H_{0 j}(x, z)=H_{j}(x, z), H_{1 j}(x, z)=\left(\frac{1-a_{0}}{2}\right) u_{1}(x, z) H_{j}(x, z) \text { e } \\
& H_{4 j}(x, z)=\left(\frac{1-a_{1}^{n_{2}} a_{2}^{n_{1}}}{2}\right) u_{4}(x, z) H_{j}(x, z) \text { para } 0 \leq j \leq 9 .
\end{aligned}
$$

- Calcule $\tilde{H}_{0 j}(x, z), \tilde{H}_{1 j}(x, z)$ e $\tilde{H}_{4 j}(x, z)$ para $0 \leq j \leq 11$ :

$$
\begin{aligned}
& \tilde{H}_{00}(x, z)=\left(\frac{1+a_{0}}{2}\right) H_{0}(x, z), \\
& \tilde{H}_{0 l}(x, z)=\left(\frac{1+a_{1}^{n_{2}} a_{2}^{n_{1}}}{2}\right) H_{l}, \\
& \tilde{H}_{0 k}(x, z)=H_{k}(x, z), \\
& \tilde{H}_{10}(x, z)=\left(\frac{1-a_{0}}{2}\right) u_{1}(x, z) H_{0}(x, z), \\
& \tilde{H}_{1 l}(x, z)=\left(\frac{1+a_{0} a_{1}^{n_{2}} a_{2}^{n_{1}}}{2}\right)\left(\frac{1-a_{0}}{2}\right) u_{1}(x, z) H_{l}(x, z), \\
& \tilde{H}_{1 k}(x, z)=0, \\
& \tilde{H}_{40}(x, z)=\left(\frac{1-a_{0}}{2}\right)\left(\frac{1-a_{1}^{n_{2}} a_{2}^{n_{1}}}{2}\right) u_{4}(x, z) H_{0}(x, z), \\
& \tilde{H}_{4 l}(x, z)=\left(\frac{1-a_{1}^{n_{2}} a_{2}^{n_{1}}}{2}\right) u_{4}(x, z) H_{l}(x, z), \\
& \tilde{H}_{4 k}(x, z)=0 \text { para } l=1,3,4,7,8,11 \text { e } k=2,5,6,9,10 .
\end{aligned}
$$

Portanto esses são os geradores para $\overrightarrow{\mathcal{Q}}_{\mathbb{R}^{2} \times \mathbb{C}^{3}}(\tilde{\Gamma})$ sobre $\mathcal{P}_{\mathbb{R}^{2} \times \mathbb{C}^{3}}(\tilde{\Gamma})$.

4. Para determinar a base de Hilbert para $\mathcal{P}_{\mathbb{R}^{2} \times \mathbb{C}^{3}}(\tilde{\Gamma})$ considere a base de Hilbert para $\mathcal{P}_{\mathbb{R}^{2} \times \mathbb{C}^{3}}\left(\tilde{\Gamma}_{+}\right)$em 5.22 e os operadores $R, S$.

Como $R\left(u_{1}\right)=\left(\frac{1+a_{0}}{2}\right) u_{1}, \quad R\left(u_{2}\right)=u_{2}, \quad R\left(u_{3}\right)=u_{3}, \quad R\left(u_{4}\right)=\left(\frac{1+a_{1}^{n_{2}} a_{2}^{n_{1}}}{2}\right) u_{4}$ , $S\left(u_{1}\right)=\tilde{u}_{1}, S\left(u_{2}\right)=S\left(u_{3}\right)=0$ e $S\left(u_{4}\right)=\tilde{u}_{4}$, segue do Teorema 1.4 .2 que

$$
\left(\frac{1+a_{0}}{2}\right) u_{1}, \quad u_{2}, \quad u_{3}, \quad\left(\frac{1+a_{1}^{n_{2}} a_{2}^{n_{1}}}{2}\right) u_{4}, \quad \tilde{u}_{1}^{2}, \quad \tilde{u}_{4}^{2}, \quad u_{6} \text { e } \tilde{u}_{1} \tilde{u}_{4} .
$$

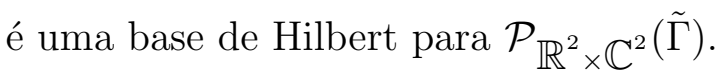

Agora, note que $a_{0}^{2}=a_{1}^{2}=a_{2}^{2}=1$. Como os geradores não dependem de $a_{3}$ então consideramos apenas as possibilidades para $a_{0}, a_{1}$ e $a_{2}$, e encontramos 4 tipos de formas normais descritos na Tabela 5.1 e com os geradores na Tabela 5.3 .

Portanto, segue o resultado: 


\begin{tabular}{|c|c|c|}
\hline & $\overrightarrow{\mathcal{Q}}_{\mathbb{R}^{2} \times \mathbb{C}^{2}(\tilde{\Gamma})}$ & $\mathcal{P}_{\mathbb{R}^{2} \times \mathbb{C}^{2}(\tilde{\Gamma})}$ \\
\hline Tipo A & $H_{j}, 0 \leq j \leq 11$ & $u_{1}, u_{2}, u_{3}, u_{4}, u_{6}$ \\
\hline Tipo B & $H_{k}, u_{4} H_{l}$ & $u_{1}, u_{2}, u_{3}, u_{4}^{2}, u_{6}$ \\
& para $k=0,2,5,6,9,10$ e $l=1,3,4,7,8,11$ & \\
\hline Tipo C & $u_{1} H_{0}, H_{k}$ para $1 \leq k \leq 11$ & $u_{1}^{2}, u_{2}, u_{3}, u_{4}, u_{6}$ \\
\hline Tipo D & $H_{k}, u_{1} H_{l}, u_{4} H_{l}$ & $u_{1}^{2}, u_{2}, u_{3}, u_{4}^{2}, u_{1} u_{4}, u_{6}$ \\
& para $k=2,5,6,9,10$ e $l=0,1,3,4,7,8,11$ & \\
\hline
\end{tabular}

Tabela 5.3: Geradores em $\mathbb{R}^{2} \times \mathbb{C}^{3}$

Teorema 5.3.4. Seja $\dot{x}=h(x)$ um sistema $\mathbf{Z}_{2}^{\phi} \times \mathbf{Z}_{2}^{\psi}$-reversível-equivariante, com $L=$ $(d h)_{0}$ definida em (5.2) para $n=3 e\left(n_{1}: n_{2}: 0\right)$-ressonante. Então, este sistema é formalmente conjugado a um dos sistemas abaixo:

Tipo A:

$$
\begin{aligned}
& \dot{x}_{1}=x_{2}+x_{1} \operatorname{Im}\left(z_{1}^{n_{2}} \bar{z}_{2}^{n_{1}}\right) f_{0}(X) \\
& \dot{x}_{2}=f_{1}(X)+x_{2} \operatorname{Im}\left(z_{1}^{n_{2}} \bar{z}_{2}^{n_{1}}\right) f_{0}(X) \\
& \dot{z}_{1}=-i \omega_{1} z_{1}+i z_{1} f_{2}(X)+i \bar{z}_{1}^{n_{2}-1} z_{2}^{n_{2}} f_{3}(X)+z_{1} \operatorname{Im}\left(z_{1}^{n_{2}} \bar{z}_{2}^{n_{1}}\right) f_{4}(X)+\bar{z}_{1}^{n_{2}-1} z_{2}^{n_{2}} \operatorname{Im}\left(z_{1}^{n_{2}} \bar{z}_{2}^{n_{1}}\right) f_{5}(X) \\
& \dot{z}_{2}=-i \omega_{2} z_{2}+i z_{2} f_{6}(X)+i z_{1}^{n_{2}} \bar{z}_{2}^{n_{1}-1} f_{7}(X)+z_{2} \operatorname{Im}\left(z_{1}^{n_{2}} \bar{z}_{2}^{n_{1}}\right) f_{8}(X)+z_{1}^{n_{2}} \bar{z}_{2}^{n_{1}-1} \operatorname{Im}\left(z_{1}^{n_{2}} \bar{z}_{2}^{n_{1}}\right) f_{9}(X), \\
& \dot{z}_{3}=-i \omega_{3} z_{3}+i z_{3} f_{10}(X)+z_{3} \operatorname{Im}\left(z_{1}^{n_{2}} \bar{z}_{2}^{n_{1}}\right) f_{11}(X) \\
& \text { para } f_{i}: \mathbb{R}^{5}, 0 \rightarrow \mathbb{R}, i=0, \ldots, 11, X=\left(x_{1},\left|z_{1}\right|^{2},\left|z_{2}\right|^{2}, \operatorname{Re}\left(z_{1}^{n_{2}} \bar{z}_{2}^{n_{1}}\right),\left|z_{3}\right|^{2}\right) .
\end{aligned}
$$

Tipo B:

$$
\begin{aligned}
& \dot{x}_{1}= x_{2}+x_{1} \operatorname{Re}\left(z_{1}^{n_{2}} \bar{z}_{2}^{n_{1}}\right) \operatorname{Im}\left(z_{1}^{n_{2}} \bar{z}_{2}^{n_{1}}\right) f_{0}(X) \\
& \dot{x}_{2}= f_{1}(X)+x_{2} \operatorname{Re}\left(z_{1}^{n_{2}} \bar{z}_{2}^{n_{1}}\right) \operatorname{Im}\left(z_{1}^{n_{2}} \bar{z}_{2}^{n_{1}}\right) f_{0}(X) \\
& \dot{z}_{1}=-i \omega_{1} z_{1}+i z_{1} f_{2}(X)+\bar{z}_{1}^{n_{2}-1} z_{2}^{n_{2}} \operatorname{Im}\left(z_{1}^{n_{2}} \bar{z}_{2}^{n_{1}}\right) f_{3}(X)+i \bar{z}_{1}^{n_{2}-1} z_{2}^{n_{2}} \operatorname{Re}\left(z_{1}^{n_{2}} \bar{z}_{2}^{n_{1}}\right) f_{4}(X)+ \\
&+z_{1} \operatorname{Re}\left(z_{1}^{n_{2}} \bar{z}_{2}^{n_{1}}\right) \operatorname{Im}\left(z_{1}^{n_{2}} \bar{z}_{2}^{n_{1}}\right) f_{5}(X) \\
& \dot{z}_{2}=-i \omega_{2} z_{2}+i z_{2} f_{6}(X)+z_{1}^{n_{2}} \bar{z}_{2}^{n_{1}-1} \operatorname{Im}\left(z_{1}^{n_{2}} \bar{z}_{2}^{n_{1}}\right) f_{7}(X)+i z_{1}^{n_{2}} \bar{z}_{2}^{n_{1}-1} \operatorname{Re}\left(z_{1}^{n_{2}} \bar{z}_{2}^{n_{1}}\right) f_{8}(X)+ \\
&+z_{2} \operatorname{Re}\left(z_{1}^{n_{2}} \bar{z}_{2}^{n_{1}}\right) \operatorname{Im}\left(z_{1}^{n_{2}} \bar{z}_{2}^{n_{1}}\right) f_{9}(X) \\
& \dot{z}_{3}=-i \omega_{3} z_{3}+i z_{3} f_{10}(X)+z_{3} \operatorname{Re}\left(z_{1}^{n_{2}} \bar{z}_{2}^{n_{1}}\right) \operatorname{Im}\left(z_{1}^{n_{2}} \bar{z}_{2}^{n_{1}}\right) f_{11}(X) \\
& \text { para } f_{i}: \mathbb{R}^{5}, 0 \rightarrow \mathbb{R}, i=0, \ldots, 11, X=\left(x_{1},\left|z_{1}\right|^{2},\left|z_{2}\right|^{2}, \operatorname{Re}^{2}\left(z_{1}^{n_{2}} \bar{z}_{2}^{n_{1}}\right),\left|z_{3}\right|^{2}\right) .
\end{aligned}
$$


Tipo $C$ :

$$
\begin{aligned}
& \dot{x}_{1}=x_{2}+x_{1} \operatorname{Im}\left(z_{1}^{n_{2}} \bar{z}_{2}^{n_{1}}\right) f_{0}(X) \\
& \dot{x}_{2}=x_{1} f_{1}(X)+x_{2} \operatorname{Im}\left(z_{1}^{n_{2}} \bar{z}_{2}^{n_{1}}\right) f_{0}(X) \\
& \dot{z}_{1}=-i \omega_{1} z_{1}+i z_{1} f_{2}(X)+i \bar{z}_{1}^{n_{2}-1} z_{2}^{n_{2}} f_{3}(X)+z_{1} \operatorname{Im}\left(z_{1}^{n_{2}} \bar{z}_{2}^{n_{1}}\right) f_{4}(X)+\bar{z}_{1}^{n_{2}-1} z_{2}^{n_{2}} \operatorname{Im}\left(z_{1}^{n_{2}} \bar{z}_{2}^{n_{1}}\right) f_{5}(X) \\
& \dot{z}_{2}=-i \omega_{2} z_{2}+i z_{2} f_{6}(X)+i z_{1}^{n_{2}} \bar{z}_{2}^{n_{1}-1} f_{7}(X)+z_{2} \operatorname{Im}\left(z_{1}^{n_{2}} \bar{z}_{2}^{n_{1}}\right) f_{8}(X)+z_{1}^{n_{2}} \bar{z}_{2}^{n_{1}-1} \operatorname{Im}\left(z_{1}^{n_{2}} \bar{z}_{2}^{n_{1}}\right) f_{9}(X) \\
& \dot{z}_{3}=-i \omega_{3} z_{3}+i z_{3} f_{10}(X)+z_{3} \operatorname{Im}\left(z_{1}^{n_{2}} \bar{z}_{2}^{n_{1}}\right) f_{11}(X) \\
& \text { para } f_{i}: \mathbb{R}^{5}, 0 \rightarrow \mathbb{R}, i=0, \ldots, 11, X=\left(x_{1}^{2},\left|z_{1}\right|^{2},\left|z_{2}\right|^{2}, \operatorname{Re}\left(z_{1}^{n_{2}} \bar{z}_{2}^{n_{1}}\right),\left|z_{3}\right|^{2}\right) .
\end{aligned}
$$

Tipo D:

$$
\begin{aligned}
\dot{x}_{1}= & \left.x_{2}+x_{1}^{2} \operatorname{Im}\left(z_{1}^{n_{2}} \bar{z}_{2}^{n_{1}}\right) f_{0}(X)+x_{1} \operatorname{Re}\left(z_{1}^{n_{2}} \bar{z}_{2}^{n_{1}}\right)\right) \operatorname{Im}\left(z_{1}^{n_{2}} \bar{z}_{2}^{n_{1}}\right) f_{1}(X) \\
\dot{x}_{2}= & \left.\left.x_{1} f_{2}(X)+x_{1} x_{2} \operatorname{Im}\left(z_{1}^{n_{2}} \bar{z}_{2}^{n_{1}}\right) f_{0}(X)+\operatorname{Re}\left(z_{1}^{n_{2}} \bar{z}_{2}^{n_{1}}\right)\right) f_{3}(X)+x_{2} \operatorname{Re}\left(z_{1}^{n_{2}} \bar{z}_{2}^{n_{1}}\right)\right) \operatorname{Im}\left(z_{1}^{n_{2}} \bar{z}_{2}^{n_{1}}\right) f_{1}(X) \\
\dot{z}_{1}= & -i \omega_{1} z_{1}+i z_{1} f_{4}(X)+\bar{z}_{1}^{n_{2}-1} z_{2}^{n_{2}} \operatorname{Im}\left(z_{1}^{n_{2}} \bar{z}_{2}^{n_{1}}\right) f_{5}(X)+i x_{1} \bar{z}_{1}^{n_{2}-1} z_{2}^{n_{2}} f_{6}(X)+ \\
& \left.\left.+x_{1} z_{1} \operatorname{Im}\left(z_{1}^{n_{2}} \bar{z}_{2}^{n_{1}}\right) f_{7}(X)+i \bar{z}_{1}^{n_{2}-1} z_{2}^{n_{2}} \operatorname{Re}\left(z_{1}^{n_{2}} \bar{z}_{2}^{n_{1}}\right)\right) f_{8}(X)+z_{1} \operatorname{Re}\left(z_{1}^{n_{2}} \bar{z}_{2}^{n_{1}}\right)\right) \operatorname{Im}\left(z_{1}^{n_{2}} \bar{z}_{2}^{n_{1}}\right) f_{9}(X) \\
\dot{z}_{2}= & -i \omega_{2} z_{2}+i z_{2} f_{10}(X)+z_{1}^{n_{2}} \bar{z}_{2}^{n_{1}-1} \operatorname{Im}\left(z_{1}^{n_{2}} \bar{z}_{2}^{n_{1}}\right) f_{11}(X)+i x_{1} z_{1}^{n_{2}} \bar{z}_{2}^{n_{1}-1} f_{12}(X)+ \\
& +x_{1} z_{2} \operatorname{Im}\left(z_{1}^{n_{2}} \bar{z}_{2}^{n_{1}}\right) f_{13}(X)+i z_{1}^{n_{2}} \bar{z}_{2}^{n_{1}-1} \operatorname{Re}\left(z_{1}^{n_{2}} \bar{z}_{2}^{n_{1}}\right) f_{14}(X)+z_{2} \operatorname{Re}\left(z_{1}^{n_{2}} \bar{z}_{2}^{n_{1}}\right) \operatorname{Im}\left(z_{1}^{n_{2}} \bar{z}_{2}^{n_{1}}\right) f_{15}(X) \\
\dot{z}_{3}= & -i \omega_{3} z_{3}+i z_{3} f_{16}(X)+x_{1} z_{3} \operatorname{Im}\left(z_{1}^{n_{2}} \bar{z}_{2}^{n_{1}}\right) f_{17}(X)+z_{3} \operatorname{Re}\left(z_{1}^{n_{2}} \bar{z}_{2}^{n_{1}}\right) \operatorname{Im}\left(z_{1}^{n_{2}} \bar{z}_{2}^{n_{1}}\right) f_{18}(X) \\
\text { para } & f_{i}: \mathbb{R}^{5}, 0 \rightarrow \mathbb{R}, i=0, \ldots, 18, X=\left(x_{1}^{2},\left|z_{1}\right|^{2},\left|z_{2}\right|^{2}, \operatorname{Re}{ }^{2}\left(z_{1}^{n_{2}} \bar{z}_{2}^{n_{1}}\right), x_{1} \operatorname{Re}\left(z_{1}^{n_{2}} \bar{z}_{2}^{n_{1}}\right),\right. \\
\left.\left|z_{3}\right|^{2}\right) . &
\end{aligned}
$$

Note que o valor de $a_{3}$ não influencia na forma normal. Isto ocorre porque $a_{3}$ está na parte de $\psi$ que age na parte algebricamente independente de $L$. Assim, o resultado desta subseção é generalizado para sistemas em $\mathbb{R}^{2} \times \mathbb{C}^{n}$ na subseção a seguir.

\subsubsection{Ressonância do tipo $\left(n_{1}: n_{2}: 0\right)$ em $\mathbb{R}^{2} \times \mathbb{C}^{n}$}

Considere o sistema (5.1), $n_{1} \omega_{2}-n_{2} \omega_{1}=0$ e $\omega_{3}, \ldots, \omega_{n}$ algebricamente independentes. Neste caso, o sistema (5.1) é chamado $\left(n_{1}: n_{2}: 0\right)$-ressonante. Note que com estas condições estamos considerando $n \geq 3$.

Consideramos coordenadas $(x, z)=\left(x_{1}, x_{2}, z_{1}, \ldots, z_{n}\right) \in \mathbb{R}^{2} \times \mathbb{C}^{n}$, temos $\mathbf{S}=\mathbf{R} \times \mathbf{T}^{n-1}$. A ação de $\mathbf{S}$ em $\mathbb{R}^{2} \times \mathbb{C}^{n}$ é conhecida através das ações de $\mathbf{R}$ em $\mathbb{R}^{2}$ dada em (5.10), e as 
ações de $\mathbf{T}$ em $\mathbb{C}^{2}$ e $\mathbb{C}$ dadas por

$$
\theta\left(z_{1}, z_{2}\right)=\left(e^{i n_{1} \theta} z_{1}, e^{i n_{2} \theta} z_{2}\right) \quad \text { e } \quad \theta z_{i}=e^{i \theta} z_{i}, 3 \leq i \leq n
$$

respectivamente.

Para este caso, também obtemos 4 tipos de formas normais (Tabela 5.1) com os geradores de $\overrightarrow{\mathcal{Q}}_{\mathbb{R}^{2} \times \mathbb{C}^{n}}\left(\mathbf{S} \rtimes\left(\mathbf{Z}^{\phi} \times \mathbf{Z}_{2}^{\psi}\right)\right)$ e $\mathcal{P}_{\mathbb{R}^{2} \times \mathbb{C}^{n}}\left(\mathbf{S} \rtimes\left(\mathbf{Z}^{\phi} \times \mathbf{Z}_{2}^{\psi}\right)\right)$ exibidos na Tabela 5.4. onde:

$$
\begin{aligned}
& u_{1}(x, z)=x_{1}, u_{2}(x, z)=\left|z_{1}\right|^{2}, u_{3}(x, z)=\left|z_{2}\right|^{2}, u_{4}(x, z)=\operatorname{Re}\left(z_{1}^{n_{2}} \bar{z}_{2}^{n_{1}}\right), u_{5}=\operatorname{Im}\left(z_{1}^{n_{2}} \bar{z}_{2}^{n_{1}}\right), \\
& u_{6}(x, z)=\left|z_{3}\right|^{2}, u_{7}(x, z)=\left|z_{4}\right|^{2}, \ldots, u_{n+3}(x, z)=\left|z_{n}\right|^{2}, \\
& H_{0}(x, z)=(0,1,0, \ldots, 0) \\
& H_{1}(x, z)=\left(x_{1} \operatorname{Im}\left(z_{1}^{n_{2}} \bar{z}_{2}^{n_{1}}\right), x_{2} \operatorname{Im}\left(z_{1}^{n_{2}} \bar{z}_{2}^{n_{1}}\right), 0, \ldots, 0\right), \\
& H_{2}(x, z)=\left(0,0, i z_{1}, 0, \ldots, 0\right) \text {, } \\
& H_{3}(x, z)=\left(0,0, i \bar{z}_{1}^{n_{2}-1} z_{2}^{n_{1}}, 0, \ldots, 0\right) \text {, } \\
& H_{4}(x, z)=\left(0,0, z_{1} \operatorname{Im}\left(z_{1}^{n_{2}} \bar{z}_{2}^{n_{1}}\right), 0, \ldots, 0\right), \\
& H_{5}(x, z)=\left(0,0, \bar{z}_{1}^{n_{2}-1} z_{2}^{n_{1}} \operatorname{Im}\left(z_{1}^{n_{2}} \bar{z}_{2}^{n_{1}}\right), 0, \ldots, 0\right), \\
& H_{6}(x, z)=\left(0,0,0, i z_{2}, 0, \ldots, 0\right) \text {, } \\
& H_{7}(x, z)=\left(0,0,0, i z_{1}^{n_{2}} \bar{z}_{2}^{n_{1}-1}, 0, \ldots, 0\right) \text {, } \\
& H_{8}(x, z)=\left(0,0,0, z_{2} \operatorname{Im}\left(z_{1}^{n_{2}} \bar{z}_{2}^{n_{1}}\right), 0, \ldots, 0\right), \\
& H_{9}(x, z)=\left(0,0,0, z_{1}^{n_{2}} \bar{z}_{2}^{n_{1}-1} \operatorname{Im}\left(z_{1}^{n_{2}} \bar{z}_{2}^{n_{1}}\right), 0, \ldots, 0\right) \text {, } \\
& H_{10}(x, z)=\left(0,0,0,0, i z_{3}, 0, \ldots, 0\right) \text {, } \\
& H_{11}(x, z)=\left(0,0,0,0, z_{3} \operatorname{Im}\left(z_{1}^{n_{2}} \bar{z}_{2}^{n_{1}}\right), 0, \ldots, 0\right), \\
& H_{12}(x, z)=\left(0,0,0,0,0, i z_{4}, \ldots, 0\right) \text {, } \\
& H_{13}(x, z)=\left(0,0,0,0,0, z_{4} \operatorname{Im}\left(z_{1}^{n_{2}} \bar{z}_{2}^{n_{1}}\right), 0, \ldots, 0\right), \\
& H_{2 n+4}(x, z)=\left(0,0,0,0, \ldots, 0, i z_{n}\right), \\
& H_{2 n+5}(x, z)=\left(0,0,0,0, \ldots, 0, z_{n} \operatorname{Im}\left(z_{1}^{n_{2}} \bar{z}_{2}^{n_{1}}\right)\right) .
\end{aligned}
$$




\begin{tabular}{|c|c|c|}
\hline & $\overrightarrow{\mathcal{Q}}_{\mathbb{R}^{2} \times \mathbb{C}^{n}(\tilde{\Gamma})}$ & $\mathcal{P}_{\mathbb{R}^{2} \times \mathbb{C}^{n}(\tilde{\Gamma})}$ \\
\hline Tipo A & $H_{j}, 0 \leq j \leq 2 n+5$ & $u_{1}, u_{2}, u_{3}, u_{4}$, \\
& $u_{6}, u_{7}, \ldots, u_{n+3}$ \\
\hline Tipo B & $H_{k}, u_{4} H_{l}$ para $k=0,2,5,6,9,10,12,14, \ldots, 2 n+4$ & $u_{1}, u_{2}, u_{3}, u_{4}^{2}$, \\
& $\mathrm{e} l=1,3,4,7,8,11,13,15, \ldots, 2 n+5$ & $u_{6}, u_{7}, \ldots, u_{n+3}$ \\
\hline Tipo C & $u_{1} H_{0}, H_{k}$ para $1 \leq k \leq 2 n+5$ & $u_{1}^{2}, u_{2}, u_{3}, u_{4}$, \\
& e $l=0,1,3,4,7,8,11,13,15, \ldots, 2 n+5$ & $u_{6}, u_{7}, \ldots, u_{n+3}$ \\
\hline Tipo D & $H_{k}, \quad u_{1} H_{l}, u_{4} H_{l}$ para $k=2,5,6,9,10,12,14, \ldots, 2 n+4$ & $u_{1}^{2}, u_{2}, u_{3}, u_{4}^{2}, u_{1} u_{4}$, \\
& & $u_{6}, u_{7}, \ldots, u_{n+3}$ \\
\hline
\end{tabular}

Tabela 5.4: Geradores em $\mathbb{R}^{2} \times \mathbb{C}^{n}$

Segue portanto, o seguinte resultado:

Teorema 5.3.5. Seja $\dot{x}=h(x)$ um sistema $\mathbf{Z}_{2}^{\phi} \times \mathbf{Z}_{2}^{\psi}$-reversivel-equivariante, com $L=$ $(d h)_{0}$ definida em (5.2) para $n \geq 3 e\left(n_{1}: n_{2}: 0\right)$-ressonante. Então, este sistema é formalmente conjugado a um dos sistemas abaixo:

Tipo A:

$$
\begin{aligned}
\dot{x}_{1}= & x_{2}+x_{1} \operatorname{Im}\left(z_{1}^{n_{2}} \bar{z}_{2}^{n_{1}}\right) f_{0}(X) \\
\dot{x}_{2}= & f_{1}(X)+x_{2} \operatorname{Im}\left(z_{1}^{n_{2}} \bar{z}_{2}^{n_{1}}\right) f_{0}(X) \\
\dot{z}_{1}= & -i \omega_{1} z_{1}+i z_{1} f_{2}(X)+i \bar{z}_{1}^{n_{2}-1} z_{2}^{n_{2}} f_{3}(X)+z_{1} \operatorname{Im}\left(z_{1}^{n_{2}} \bar{z}_{2}^{n_{1}}\right) f_{4}(X)+ \\
& +\bar{z}_{1}^{n_{2}-1} z_{2}^{n_{2}} \operatorname{Im}\left(z_{1}^{n_{2}} \bar{z}_{2}^{n_{1}}\right) f_{5}(X) \\
\dot{z}_{2}= & -i \omega_{2} z_{2}+i z_{2} f_{6}(X)+i z_{1}^{n_{2}} \bar{z}_{2}^{n_{1}-1} f_{7}(X)+z_{2} \operatorname{Im}\left(z_{1}^{n_{2}} \bar{z}_{2}^{n_{1}}\right) f_{8}(X)+ \\
& +z_{1}^{n_{2}} \bar{z}_{2}^{n_{1}-1} \operatorname{Im}\left(z_{1}^{n_{2}} \bar{z}_{2}^{n_{1}}\right) f_{9}(X), \\
\dot{z}_{3}= & -i \omega_{3} z_{3}+i z_{3} f_{10}(X)+z_{3} \operatorname{Im}\left(z_{1}^{n_{2}} \bar{z}_{2}^{n_{1}}\right) f_{11}(X) \\
\vdots & \\
\dot{z}_{n}= & -i \omega_{n} z_{n}+i z_{n} f_{2 n+4}(X)+z_{n} \operatorname{Im}\left(z_{1}^{n_{2}} \bar{z}_{2}^{n_{1}}\right) f_{2 n+5}(X)
\end{aligned}
$$

para $f_{i}: \mathbb{R}^{n+2}, 0 \rightarrow \mathbb{R}, 0 \leq i \leq 2 n+5, X=\left(x_{1},\left|z_{1}\right|^{2},\left|z_{2}\right|^{2}, \operatorname{Re}\left(z_{1}^{n_{2}} \bar{z}_{2}^{n_{1}}\right),\left|z_{3}\right|^{2}, \ldots,\left|z_{n}\right|^{2}\right)$ 
Tipo B:

$$
\begin{aligned}
\dot{x}_{1}= & x_{2}+x_{1} \operatorname{Re}\left(z_{1}^{n_{2}} \bar{z}_{2}^{n_{1}}\right) \operatorname{Im}\left(z_{1}^{n_{2}} \bar{z}_{2}^{n_{1}}\right) f_{0}(X) \\
\dot{x}_{2}= & f_{1}(X)+x_{2} \operatorname{Re}\left(z_{1}^{n_{2}} \bar{z}_{2}^{n_{1}}\right) \operatorname{Im}\left(z_{1}^{n_{2}} \bar{z}_{2}^{n_{1}}\right) f_{0}(X) \\
\dot{z}_{1}= & -i \omega_{1} z_{1}+i z_{1} f_{2}(X)+\bar{z}_{1}^{n_{2}-1} z_{2}^{n_{2}} \operatorname{Im}\left(z_{1}^{n_{2}} \bar{z}_{2}^{n_{1}}\right) f_{3}(X)+i \bar{z}_{1}^{n_{2}-1} z_{2}^{n_{2}} \operatorname{Re}\left(z_{1}^{n_{2}} \bar{z}_{2}^{n_{1}}\right) f_{4}(X)+ \\
& +z_{1} \operatorname{Re}\left(z_{1}^{n_{2}} \bar{z}_{2}^{n_{1}}\right) \operatorname{Im}\left(z_{1}^{n_{2}} \bar{z}_{2}^{n_{1}}\right) f_{5}(X) \\
\dot{z}_{2}= & -i \omega_{2} z_{2}+i z_{2} f_{6}(X)+z_{1}^{n_{2}} \bar{z}_{2}^{n_{1}-1} \operatorname{Im}\left(z_{1}^{n_{2}} \bar{z}_{2}^{n_{1}}\right) f_{7}(X)+i z_{1}^{n_{2}} \bar{z}_{2}^{n_{1}-1} \operatorname{Re}\left(z_{1}^{n_{2}} \bar{z}_{2}^{n_{1}}\right) f_{8}(X)+ \\
& +z_{2} \operatorname{Re}\left(z_{1}^{n_{2}} \bar{z}_{2}^{n_{1}}\right) \operatorname{Im}\left(z_{1}^{n_{2}} \bar{z}_{2}^{n_{1}}\right) f_{9}(X) \\
\dot{z}_{3}= & -i \omega_{3} z_{3}+i z_{3} f_{10}(X)+z_{3} \operatorname{Re}\left(z_{1}^{n_{2}} \bar{z}_{2}^{n_{1}}\right) \operatorname{Im}\left(z_{1}^{n_{2}} \bar{z}_{2}^{n_{1}}\right) f_{11}(X) \\
\vdots & \\
\dot{z}_{n}= & -i \omega_{n} z_{n}+i z_{n} f_{2 n+4}(X)+z_{n} \operatorname{Re}\left(z_{1}^{n_{2}} \bar{z}_{2}^{n_{1}}\right) \operatorname{Im}\left(z_{1}^{n_{2}} \bar{z}_{2}^{n_{1}}\right) f_{2 n+5}(X)
\end{aligned}
$$

para $f_{i}: \mathbb{R}^{n+2}, 0 \rightarrow \mathbb{R}, 0 \leq i \leq 2 n+5, \quad X=\left(x_{1},\left|z_{1}\right|^{2},\left|z_{2}\right|^{2}, \operatorname{Re}^{2}\left(z_{1}^{n_{2}} \bar{z}_{2}^{n_{1}}\right),\left|z_{3}\right|^{2}, \ldots,\left|z_{n}\right|^{2}\right)$.

Tipo C:

$$
\begin{aligned}
\dot{x}_{1}= & x_{2}+x_{1} \operatorname{Im}\left(z_{1}^{n_{2}} \bar{z}_{2}^{n_{1}}\right) f_{0}(X) \\
\dot{x}_{2}= & x_{1} f_{1}(X)+x_{2} \operatorname{Im}\left(z_{1}^{n_{2}} \bar{z}_{2}^{n_{1}}\right) f_{0}(X) \\
\dot{z}_{1}= & -i \omega_{1} z_{1}+i z_{1} f_{2}(X)+i \bar{z}_{1}^{n_{2}-1} z_{2}^{n_{2}} f_{3}(X)+z_{1} \operatorname{Im}\left(z_{1}^{n_{2}} \bar{z}_{2}^{n_{1}}\right) f_{4}(X)+ \\
& +\bar{z}_{1}^{n_{2}-1} z_{2}^{n_{2}} \operatorname{Im}\left(z_{1}^{n_{2}} \bar{z}_{2}^{n_{1}}\right) f_{5}(X) \\
\dot{z}_{2}= & -i \omega_{2} z_{2}+i z_{2} f_{6}(X)+i z_{1}^{n_{2}} \bar{z}_{2}^{n_{1}-1} f_{7}(X)+z_{2} \operatorname{Im}\left(z_{1}^{n_{2}} \bar{z}_{2}^{n_{1}}\right) f_{8}(X)+ \\
& +z_{1}^{n_{2}} \bar{z}_{2}^{n_{1}-1} \operatorname{Im}\left(z_{1}^{n_{2}} \bar{z}_{2}^{n_{1}}\right) f_{9}(X) \\
\dot{z}_{3}= & -i \omega_{3} z_{3}+i z_{3} f_{10}(X)+z_{3} \operatorname{Im}\left(z_{1}^{n_{2}} \bar{z}_{2}^{n_{1}}\right) f_{11}(X) \\
\vdots & \\
\dot{z}_{3}= & -i \omega_{n} z_{n}+i z_{n} f_{2 n+4}(X)+z_{n} \operatorname{Im}\left(z_{1}^{n_{2}} \bar{z}_{2}^{n_{1}}\right) f_{2 n+5}(X)
\end{aligned}
$$

para $f_{i}: \mathbb{R}^{n+2}, 0 \rightarrow \mathbb{R}, 0 \leq i \leq 2 n+5, \quad X=\left(x_{1}^{2},\left|z_{1}\right|^{2},\left|z_{2}\right|^{2}, \operatorname{Re}\left(z_{1}^{n_{2}} \bar{z}_{2}^{n_{1}}\right),\left|z_{3}\right|^{2}, \ldots,\left|z_{n}\right|^{2}\right)$ 
Tipo D:

$$
\begin{aligned}
& \dot{x}_{1}=\left.x_{2}+x_{1}^{2} \operatorname{Im}\left(z_{1}^{n_{2}} \bar{z}_{2}^{n_{1}}\right) f_{0}(X)+x_{1} \operatorname{Re}\left(z_{1}^{n_{2}} \bar{z}_{2}^{n_{1}}\right)\right) \operatorname{Im}\left(z_{1}^{n_{2}} \bar{z}_{2}^{n_{1}}\right) f_{1}(X) \\
& \dot{x}_{2}=\left.\left.x_{1} f_{2}(X)+x_{1} x_{2} \operatorname{Im}\left(z_{1}^{n_{2}} \bar{z}_{2}^{n_{1}}\right) f_{0}(X)+\operatorname{Re}\left(z_{1}^{n_{2}} \bar{z}_{2}^{n_{1}}\right)\right) f_{3}(X)+x_{2} \operatorname{Re}\left(z_{1}^{n_{2}} \bar{z}_{2}^{n_{1}}\right)\right) \operatorname{Im}\left(z_{1}^{n_{2}} \bar{z}_{2}^{n_{1}}\right) f_{1}(X) \\
& \dot{z}_{1}=-i \omega_{1} z_{1}+i z_{1} f_{4}(X)+\bar{z}_{1}^{n_{2}-1} z_{2}^{n_{2}} \operatorname{Im}\left(z_{1}^{n_{2}} \bar{z}_{2}^{n_{1}}\right) f_{5}(X)+i x_{1} \bar{z}_{1}^{n_{2}-1} z_{2}^{n_{2}} f_{6}(X)+ \\
&\left.\left.+x_{1} z_{1} \operatorname{Im}\left(z_{1}^{n_{2}} \bar{z}_{2}^{n_{1}}\right) f_{7}(X)+i \bar{z}_{1}^{n_{2}-1} z_{2}^{n_{2}} \operatorname{Re}\left(z_{1}^{n_{2}} \bar{z}_{2}^{n_{1}}\right)\right) f_{8}(X)+z_{1} \operatorname{Re}\left(z_{1}^{n_{2}} \bar{z}_{2}^{n_{1}}\right)\right) \operatorname{Im}\left(z_{1}^{n_{2}} \bar{z}_{2}^{n_{1}}\right) f_{9}(X) \\
& \dot{z}_{2}=-i \omega_{2} z_{2}+i z_{2} f_{10}(X)+z_{1}^{n_{2}} \bar{z}_{2}^{n_{1}-1} \operatorname{Im}\left(z_{1}^{n_{2}} \bar{z}_{2}^{n_{1}}\right) f_{11}(X)+i x_{1} z_{1}^{n_{2}} \bar{z}_{2}^{n_{1}-1} f_{12}(X)+ \\
&+x_{1} z_{2} \operatorname{Im}\left(z_{1}^{n_{2}} \bar{z}_{2}^{n_{1}}\right) f_{13}(X)+i z_{1}^{n_{2}} \bar{z}_{2}^{n_{1}-1} \operatorname{Re}\left(z_{1}^{n_{2}} \bar{z}_{2}^{n_{1}}\right) f_{14}(X)+z_{2} \operatorname{Re}\left(z_{1}^{n_{2}} \bar{z}_{2}^{n_{1}}\right) \operatorname{Im}\left(z_{1}^{n_{2}} \bar{z}_{2}^{n_{1}}\right) f_{15}(X) \\
& \dot{z}_{3}=-i \omega_{2} z_{3}+i z_{3} f_{16}(X)+x_{1} z_{3} \operatorname{Im}\left(z_{1}^{n_{2}} \bar{z}_{2}^{n_{1}}\right) f_{17}(X)+z_{3} \operatorname{Re}\left(z_{1}^{n_{2}} \bar{z}_{2}^{n_{1}}\right) \operatorname{Im}\left(z_{1}^{n_{2}} \bar{z}_{2}^{n_{1}}\right) f_{18}(X) \\
& \vdots \\
& \dot{z}_{n}=-i \omega_{n} z_{n}+i z_{n} f_{3 n+7}(X)+x_{1} z_{n} \operatorname{Im}\left(z_{1}^{n_{2}} \bar{z}_{2}^{n_{1}}\right) f_{3 n+8}(X)+z_{n} \operatorname{Re}\left(z_{1}^{n_{2}} \bar{z}_{2}^{n_{1}}\right) \operatorname{Im}\left(z_{1}^{n_{2}} \bar{z}_{2}^{n_{1}}\right) f_{3 n+9}(X), \\
& \text { para } f_{i}: \mathbb{R}^{n+3}, 0 \rightarrow \mathbb{R}, 0 \leq i \leq 3 n+9, \quad X=\left(x_{1}^{2},\left|z_{1}\right|^{2},\left|z_{2}\right|^{2}, \operatorname{Re}^{2}\left(z_{1}^{n_{2}} \bar{z}_{2}^{n_{1}}\right), x_{1} \operatorname{Re}\left(z_{1}^{n_{2}} \bar{z}_{2}^{n_{1}}\right),\right. \\
&\left.\left|z_{3}\right|^{2}, \ldots,\left|z_{n}\right|^{2}\right) .
\end{aligned}
$$

\subsubsection{Ressonância do tipo $\left(n_{1}: n_{2}-m_{1}: m_{2}\right)$ em $\mathbb{R}^{2} \times \mathbb{C}^{4}$}

Nesta Subseção considere o sistema (5.1), com $n_{1} \omega_{2}-n_{2} \omega_{1}=0$ e $m_{1} \omega_{4}-m_{2} \omega_{3}=0$. Sob estas condições, o sistema (5.1) é chamado $\left(n_{1}: n_{2}: m_{1}: m_{2}\right)$-ressonante.

Assim, $\mathbf{S}=\mathbf{R} \times \mathbf{T} \times \mathbf{T}$ e a ação de $\mathbf{S}$ em $\mathbb{R}^{2} \times \mathbb{C}^{4}$ é determinada através das ações de $\mathbf{R}$ em $\mathbb{R}^{2}$ dada em 5.10 , e pelas ações de $\mathbf{T}$ em $\mathbb{C}^{2}$ dadas por

$$
\theta\left(z_{1}, z_{2}\right)=\left(e^{i n_{1} \theta} z_{1}, e^{i n_{2} \theta} z_{2}\right) \text { e } \theta\left(z_{3}, z_{4}\right)=\left(e^{i m_{1} \theta} z_{3}, e^{i m_{2} \theta} z_{4}\right)
$$

em coordenadas complexas $(x, z)=\left(x_{1}, x_{2}, z_{1}, z_{2}, z_{3}, z_{4}\right) \in \mathbb{R}^{2} \times \mathbb{C}^{4}$.

Geradores para os invariantes e equivariantes para estas ações são dados como em (5.18) e 5.19).

Considere ainda as ações de $\mathbf{Z}_{2}^{\phi}$ e $\mathbf{Z}_{2}^{\psi}$ em $\mathbb{R}^{2} \times \mathbb{C}^{4}$ dadas em 5.11) e 5.12), respectivamente para $n=4$. Seja $\tilde{\Gamma}=\mathbf{S} \rtimes\left(\mathbf{Z}_{2}^{\phi} \times \mathbf{Z}_{2}^{\psi}\right)$. Devemos determinar um conjunto de geradores para $\overrightarrow{\mathcal{Q}}_{\mathbb{R}^{2} \times \mathbb{C}^{4}}(\tilde{\Gamma})$ sobre $\mathcal{P}_{\mathbb{R}^{2} \times \mathbb{C}^{4}}(\tilde{\Gamma})$ :

1. Seja $\Gamma=\mathbf{S} \rtimes \mathbf{Z}_{2}^{\phi}$ e fixe a antissimetria $\delta=\phi$. Temos $\Gamma_{+}=\mathbf{S}$. Considere os geradores para os invariantes e equivariantes dados em (5.13), (5.18) e 5.19). Pelo Lema 2.2.1.

$$
u_{1}(x, z)=x_{1}, u_{2}(x, z)=\left|z_{1}\right|^{2}, u_{3}(x, z)=\left|z_{2}\right|^{2}, u_{4}(x, z)=\operatorname{Re}\left(z_{1}^{n_{2}} \bar{z}_{2}^{n_{1}}\right),
$$




$$
\begin{gathered}
u_{5}(x, z)=\operatorname{Im}\left(z_{1}^{n_{2}} \bar{z}_{2}^{n_{1}}\right), u_{6}(x, z)=\left|z_{3}\right|^{2}, u_{7}(x, z)=\left|z_{4}\right|^{2}, \\
u_{8}(x, z)=\operatorname{Re}\left(z_{3}^{m_{2}} \bar{z}_{4}^{m_{1}}\right) \text { e } u_{9}(x, z)=\operatorname{Im}\left(z_{3}^{m_{2}} \bar{z}_{4}^{m_{1}}\right),
\end{gathered}
$$

é uma base de Hilbert para $\mathcal{P}_{\mathbb{R}^{2} \times \mathbb{C}^{3}}\left(\Gamma_{+}\right)$.

Do Lema 2.2 .2 segue que os geradores para $\overrightarrow{\mathcal{P}}_{\mathbb{R}^{2} \times \mathbb{C}^{3}}\left(\Gamma_{+}\right)$sobre $\mathcal{P}_{\mathbb{R}^{2} \times \mathbb{C}^{2}}\left(\Gamma_{+}\right)$são dados por:

$$
\begin{aligned}
& H_{0}(x, z)=\left(x_{1}, x_{2}, 0,0,0,0\right), \\
& H_{1}(x, z)=(0,1,0,0,0,0) \text {, } \\
& H_{2}(x, z)=\left(0,0, z_{1}, 0,0,0\right) \text {, } \\
& H_{3}(x, z)=\left(0,0, i z_{1}, 0,0,0\right) \text {, } \\
& H_{4}(x, z)=\left(0,0, \bar{z}_{1}^{n_{2}-1} z_{2}^{n_{1}}, 0,0,0\right), \\
& H_{5}(x, z)=\left(0,0, i \bar{z}_{1}^{n_{2}-1} z_{2}^{n_{1}}, 0,0,0\right), \\
& H_{6}(x, z)=\left(0,0,0, z_{2}, 0,0\right), \\
& H_{7}(x, z)=\left(0,0,0, i z_{2}, 0,0\right), \\
& H_{8}(x, z)=\left(0,0,0, z_{1}^{n_{2}} \bar{z}_{2}^{n_{1}-1}, 0,0\right), \\
& H_{9}(x, z)=\left(0,0,0, i z_{1}^{n_{2}} \bar{z}_{2}^{n_{1}-1}, 0,0\right), \\
& H_{10}(x, z)=\left(0,0,0,0, z_{3}, 0\right) \text {, } \\
& H_{11}(x, z)=\left(0,0,0,0, i z_{3}, 0\right) \text {. } \\
& H_{12}(x, z)=\left(0,0,0,0, \bar{z}_{3}^{m_{2}-1} z_{4}^{m_{1}}, 0\right) \text {, } \\
& H_{13}(x, z)=\left(0,0,0,0, i \bar{z}_{3}^{m_{2}-1} z_{4}^{m_{1}}, 0\right) \text {, } \\
& H_{14}(x, z)=\left(0,0,0,0,0, z_{4}\right), \\
& H_{15}(x, z)=\left(0,0,0,0,0, i z_{4}\right), \\
& H_{16}(x, z)=\left(0,0,0,0, z_{3}^{m_{2}} \bar{z}_{4}^{m_{1}-1}\right) \text {, } \\
& H_{17}(x, z)=\left(0,0,0,0, i z_{3}^{m_{2}} \bar{z}_{4}^{m_{1}-1}\right)
\end{aligned}
$$

2. Agora, aplicamos o Algoritmo 1.4 .1 e obtemos geradores para $\overrightarrow{\mathcal{Q}}_{\mathbb{R}^{2} \times \mathbb{C}^{3}}(\Gamma)$ sobre $\mathcal{P}_{\mathbb{R}^{2} \times \mathbb{C}^{3}}(\Gamma)$ :

$$
H_{i}(x, z), \quad u_{5}(x, z) H_{j}(x, z) \text { e } u_{9}(x, z) H_{j}(x, z)
$$


para $i=1,3,5,7,9,11,13,15,17$ e $j=0,2,4,6,8,10,12,14,16$.

Usando os operadores de Reynolds $R, S$ e o Teorema 1.4.2, obtemos uma base de Hilbert para $\mathcal{P}_{\mathbb{R}^{2} \times \mathbb{C}^{2}}(\Gamma)$ e usamos uma outra notação para não haver dúvidas nos passos seguintes:

$$
\begin{gathered}
v_{1}=u_{1}, \quad v_{2}=u_{2}, \quad v_{3}=u_{3}, \quad v_{4}=u_{4}, \quad v_{5}=u_{6}, \\
v_{6}=u_{7}, \quad v_{7}=u_{8} \quad \text { e } \quad v_{8}=u_{5} u_{9},
\end{gathered}
$$

uma vez que $R\left(u_{i}\right)=u_{i}, \quad R\left(u_{5}\right)=R\left(u_{9}\right)=0, \quad S\left(u_{j}\right)=0, \quad S\left(u_{5}\right)=u_{5} \quad \mathrm{e}$ $S\left(u_{9}\right)=u_{9}$ para $i=1,2,3,4,6,7,8$ e $j=1,2,3,4,6$. Como $u_{5}^{2}$ e $u_{9}^{2}$ são obtidos de (5.25), ou seja, $u_{5}^{2}=u_{2}^{n_{2}} u_{3}^{n_{1}}-u_{4}^{2}$ e $u_{9}^{2}=u_{6}^{m_{2}} u_{7}^{m_{1}}-u_{8}^{2}$, o resultado segue.

3. Agora, seja $\tilde{\Gamma}=\mathbf{S} \rtimes\left(\mathbf{Z}_{2}^{\phi} \times \mathbf{Z}_{2}^{\psi}\right)$ e fixe a antissimetria $\delta=\psi$. Considere a representação dual de $\mathbf{Z}_{2}^{\phi}$ em $\mathbb{R}^{2} \times \mathbb{C}^{4}$. Então, $\tilde{\Gamma}_{+}=\mathbf{S} \rtimes \mathbf{Z}_{2}^{\phi}$.

Considere os geradores para $\mathcal{P}_{\mathbb{R}^{2} \times \mathbb{C}^{3}}\left(\tilde{\Gamma}_{+}\right)$e $\overrightarrow{\mathcal{P}}_{\mathbb{R}^{2} \times \mathbb{C}^{3}}\left(\tilde{\Gamma}_{+}\right)$calculados no item 2 e aplique o Algoritmo 1.4.1.

- Tome $\tilde{v}_{0}(x, z)=1$. Faça

$$
\begin{aligned}
& \tilde{v}_{1}(x, z)=\left(\frac{1-a_{0}}{2}\right) v_{1}(x, z), \\
& \tilde{v}_{2}(x, z)=\tilde{v}_{3}(x, z)=\tilde{v}_{5}(x, z)=\tilde{v}_{6}(x, z)=0, \\
& \tilde{v}_{4}(x, z)=\left(\frac{1-a_{1}^{n_{2}} a_{2}^{n_{1}}}{2}\right) v_{4}(x, z), \\
& \tilde{v}_{7}(x, z)=\left(\frac{1-a_{3}^{m_{2}} a_{4}^{m_{1}}}{2}\right) v_{7}(x, z), \\
& \tilde{v}_{8}(x, z)=\left(\frac{1-a_{1}^{n_{2}} a_{2}^{n_{1}} a_{3}^{m_{2}} a_{4}^{m_{1}}}{2}\right) v_{8}(x, z) .
\end{aligned}
$$

- Faça

$$
\begin{aligned}
& J_{p i}(x, z)=\tilde{v}_{p}(x, z) H_{i}(x, z) \\
& J_{p 5 j}(x, z)=\tilde{v}_{p}(x, z) u_{5}(x, z) H_{j}(x, z), \\
& J_{p 9 j}(x, z)=\tilde{v}_{p}(x, z) u_{9}(x, z) H_{j}(x, z), \\
& \text { para } i=1,3,5,7,9,11,13,15,17, j=0,2,4,6,8,10,12,14,16 \quad \text { e } p= \\
& 0,1,4,7,8 .
\end{aligned}
$$

- Calcule $\tilde{J}_{p q}=\frac{1}{2}\left(J_{p i}-\delta^{-1} J_{p q} \delta\right)$ para $p=0,1,4,7,8$ e $0 \leq q \leq 17$ :

$$
\tilde{J}_{01}(x, z)=\left(\frac{1+a_{0}}{2}\right) H_{1}(x, z)
$$




$$
\begin{aligned}
& \tilde{J}_{0 i}(x, z)=H_{i}(x, z), \\
& \tilde{J}_{0 k}(x, z)=\left(\frac{1+a_{1}^{n_{2}} a_{2}^{n_{1}}}{2}\right) H_{k}(x, z), \\
& \tilde{J}_{0 l}(x, z)=\left(\frac{1+a_{3}^{m_{2}} a_{4}^{m_{1}}}{2}\right) H_{l}(x, z), \\
& \tilde{J}_{11}(x, z)=\left(\frac{1-a_{0}}{2}\right) v_{1}(x, z) H_{1}(x, z), \\
& \tilde{J}_{1 i}(x, z)=0 \text {, } \\
& \tilde{J}_{1 k}(x, z)=\left(\frac{1-a_{0}}{2}\right)\left(\frac{1-a_{1}^{n_{2}} a_{2}^{n_{1}}}{2}\right) v_{1}(x, z) H_{k}(x, z), \\
& \tilde{J}_{1 l}(x, z)=\left(\frac{1-a_{0}}{2}\right)\left(\frac{1-a_{3}^{m_{2}} a_{4}^{m_{1}}}{2}\right) v_{1}(x, z) H_{l}(x, z), \\
& \tilde{J}_{41}(x, z)=\left(\frac{1-a_{0}}{2}\right)\left(\frac{1-a_{1}^{n_{2}} a_{2}^{n_{1}}}{2}\right) v_{4}(x, z) H_{1}(x, z) \text {, } \\
& \tilde{J}_{4 i}(x, z)=0 \text {, } \\
& \tilde{J}_{4 k}(x, z)=\left(\frac{1-a_{1}^{n_{2}} a_{2}^{n_{1}}}{2}\right) v_{4}(x, z) H_{k}(x, z), \\
& \tilde{J}_{4 l}(x, z)=\left(\frac{1-a_{1}^{n_{2}} a_{2}^{n_{1}}}{2}\right)\left(\frac{1-a_{3}^{m_{2}} a_{4}^{m_{1}}}{2}\right) v_{4}(x, z) H_{l}(x, z), \\
& \tilde{J}_{71}(x, z)=\left(\frac{1-a_{0}}{2}\right)\left(\frac{1-a_{3}^{m_{2}} a_{4}^{m_{1}}}{2}\right) v_{7}(x, z) H_{1}(x, z), \\
& \tilde{J}_{7 i}(x, z)=0 \\
& \tilde{J}_{7 k}(x, z)=\left(\frac{1-a_{1}^{n_{2}} a_{2}^{n_{1}}}{2}\right)\left(\frac{1-a_{3}^{m_{2}} a_{4}^{m_{1}}}{2}\right) v_{7}(x, z) H_{k}(x, z), \\
& \tilde{J}_{7 l}(x, z)=\left(\frac{1-a_{3}^{m_{2}} a_{4}^{m_{1}}}{2}\right) v_{7}(x, z) H_{l}(x, z), \\
& \tilde{J}_{81}(x, z)=\left(\frac{1-a_{0}}{2}\right)\left(\frac{1-a_{1}^{n_{2}} a_{2}^{n_{1}} a_{3}^{m_{2}} a_{4}^{m_{1}}}{2}\right) v_{8}(x, z) H_{1}(x, z), \\
& \tilde{J}_{8 k}(x, z)=\left(\frac{1-a_{1}^{n_{2}} a_{2}^{n_{1}}}{2}\right)\left(\frac{1-a_{1}^{n_{2}} a_{2}^{n_{1}} a_{3}^{m_{2}} a_{4}^{m_{1}}}{2}\right) v_{8}(x, z) H_{k}(x, z), \\
& \tilde{J}_{8 i}(x, z)=0 \text {, } \\
& \tilde{J}_{8 l}(x, z)=\left(\frac{1-a_{3}^{m_{2}} a_{4}^{m_{1}}}{2}\right)\left(\frac{1-a_{1}^{n_{2}} a_{2}^{n_{1}} a_{3}^{m_{2}} a_{4}^{m_{1}}}{2}\right) v_{8}(x, z) H_{l}(x, z) \text {, } \\
& \tilde{J}_{05 j}(x, z)=\left(\frac{1+a_{1}^{n_{2}} a_{2}^{n_{1}}}{2}\right) u_{5}(x, z) H_{j}(x, z) \text {, } \\
& \tilde{J}_{05 r}(x, z)=u_{5}(x, z) H_{r}(x, z) \text {, } \\
& \tilde{J}_{05 s}(x, z)=\left(\frac{1+a_{1}^{n_{2}} a_{2}^{n_{1}} a_{3}^{m_{2}} a_{4}^{m_{1}}}{2}\right) u_{5}(x, z) H_{s}(x, z) \text {, } \\
& \tilde{J}_{15 j}(x, z)=\left(\frac{1-a_{0}}{2}\right)\left(\frac{1+a_{1}^{n_{2}} a_{2}^{n_{1}}}{2}\right) v_{1}(x, z) u_{5}(x, z) H_{s}(x, z), \\
& \tilde{J}_{15 r}(x, z)=\left(\frac{1-a_{0}}{2}\right) v_{1}(x, z) u_{5}(x, z) H_{r}(x, z),
\end{aligned}
$$




$$
\begin{aligned}
& \tilde{J}_{15 s}(x, z)=\left(\frac{1-a_{0}}{2}\right)\left(\frac{1+a_{1}^{n_{2}} a_{2}^{n_{1}} a_{3}^{m_{2}} a_{4}^{m_{1}}}{2}\right) v_{1}(x, z) u_{5}(x, z) H_{s}(x, z), \\
& \tilde{J}_{45 j}(x, z)=\left(\frac{1-a_{1}^{n_{2}} a_{2}^{n_{1}}}{2}\right) v_{4}(x, z) u_{5}(x, z) H_{j}(x, z) \text {, } \\
& \tilde{J}_{45 r}(x, z)=0 \text {, } \\
& \tilde{J}_{45 s}(x, z)=\left(\frac{1-a_{1}^{n_{2}} a_{2}^{n_{1}}}{2}\right)\left(\frac{1-a_{1}^{n_{2}} a_{2}^{n_{1}} a_{3}^{m_{2}} a_{4}^{m_{1}}}{2}\right) v_{4}(x, z) u_{5}(x, z) H_{s}(x, z) \text {, } \\
& \tilde{J}_{75 j}(x, z)=\left(\frac{1-a_{1}^{n_{2}} a_{2}^{n_{1}}}{2}\right)\left(\frac{1-a_{3}^{m_{2}} a_{4}^{m_{1}}}{2}\right) u_{5}(x, z) v_{7}(x, z) H_{j}(x, z), \\
& \tilde{J}_{75 r}(x, z)=0 \text {, } \\
& \tilde{J}_{75 s}(x, z)=\left(\frac{1-a_{3}^{m_{2}} a_{4}^{m_{1}}}{2}\right)\left(\frac{1-a_{1}^{n_{2}} a_{2}^{n_{1}} a_{3}^{m_{2}} a_{4}^{m_{1}}}{2}\right) u_{5}(x, z) v_{7}(x, z) H_{s}(x, z) \text {, } \\
& \tilde{J}_{85 j}(x, z)=\left(\frac{1-a_{1}^{n_{2}} a_{2}^{n_{1}}}{2}\right)\left(\frac{1-a_{1}^{n_{2}} a_{2}^{n_{1}} a_{3}^{m_{2}} a_{4}^{m_{1}}}{2}\right) u_{5}(x, z) v_{8}(x, z) H_{j}(x, z), \\
& \tilde{J}_{85 r}(x, z)=0 \text {, } \\
& \tilde{J}_{85 s}(x, z)=\left(\frac{1-a_{1}^{n_{2}} a_{2}^{n_{1}} a_{3}^{m_{2}} a_{4}^{m_{1}}}{2}\right) u_{5}(x, z) v_{8}(x, z) H_{s}(x, z), \\
& \tilde{J}_{09 j}(x, z)=\left(\frac{1+a_{3}^{m_{2}} a_{4}^{m_{1}}}{2}\right) u_{9}(x, z) H_{j}(x, z), \\
& \tilde{J}_{09 s}(x, z)=u_{9}(x, z) H_{j}(x, z) \text {, } \\
& \tilde{J}_{09 r}(x, z)=\left(\frac{1+a_{1}^{n_{2}} a_{2}^{n_{1}} a_{3}^{m_{2}} a_{4}^{m_{1}}}{2}\right) u_{9}(x, z) H_{r}(x, z), \\
& \tilde{J}_{19 j}(x, z)=\left(\frac{1-a_{0}}{2}\right)\left(\frac{1+a_{3}^{m_{2}} a_{4}^{m_{1}}}{2}\right) v_{1}(x, z) u_{9}(x, z) H_{j}(x, z), \\
& \tilde{J}_{19 r}(x, z)=\left(\frac{1-a_{0}}{2}\right)\left(\frac{1+a_{1}^{n_{2}} a_{2}^{n_{1}} a_{3}^{m_{2}} a_{4}^{m_{1}}}{2}\right) v_{1}(x, z) u_{9}(x, z) H_{r}(x, z) \text {, } \\
& \tilde{J}_{19 s}(x, z)=\left(\frac{1-a_{0}}{2}\right) v_{1}(x, z) u_{9}(x, z) H_{s}(x, z) \text {, } \\
& \tilde{J}_{49 j}(x, z)=\left(\frac{1-a_{3}^{m_{2}} a_{4}^{m_{1}}}{2}\right) v_{4}(x, z) u_{9}(x, z) H_{j}(x, z), \\
& \tilde{J}_{49 r}(x, z)=\left(\frac{1-a_{1}^{n_{2}} a_{2}^{n_{1}} a_{3}^{m_{2}} a_{4}^{m_{1}}}{2}\right) v_{4}(x, z) u_{9}(x, z) H_{r}(x, z), \\
& \tilde{J}_{49 r}(x, z)=0 \text {, } \\
& \tilde{J}_{79 j}(x, z)=\left(\frac{1-a_{3}^{m_{2}} a_{4}^{m_{1}}}{2}\right) v_{7}(x, z) u_{9}(x, z) H_{j}(x, z) \text {, } \\
& \tilde{J}_{79 r}(x, z)=\left(\frac{1-a_{3}^{m_{2}} a_{4}^{m_{1}}}{2}\right)\left(\frac{1-a_{1}^{n_{2}} a_{2}^{n_{1}} a_{3}^{m_{2}} a_{4}^{m_{1}}}{2}\right) v_{7}(x, z) u_{9}(x, z) H_{r}(x, z), \\
& \tilde{J}_{79 s}(x, z)=0 \text {, } \\
& \tilde{J}_{89 j}(x, z)=\left(\frac{1-a_{3}^{m_{2}} a_{4}^{m_{1}}}{2}\right)\left(\frac{1-a_{1}^{n_{2}} a_{2}^{n_{1}} a_{3}^{m_{2}} a_{4}^{m_{1}}}{2}\right) v_{8}(x, z) u_{9}(x, z) H_{j}(x, z) \text {, } \\
& \tilde{J}_{89 r}(x, z)=\left(\frac{1-a_{1}^{n_{2}} a_{2}^{n_{1}} a_{3}^{m_{2}} a_{4}^{m_{1}}}{2}\right) v_{8}(x, z) u_{9}(x, z) H_{r}(x, z),
\end{aligned}
$$




$$
\begin{aligned}
& \tilde{J}_{89 s}(x, z)=0, \\
& \text { para } i=3,7,11,15, \quad j=0,2,6,10,14, \quad k=5,9, \quad l=13,17, \quad r=4,8 \quad \mathrm{e} \\
& s=12,16 .
\end{aligned}
$$

Estes são os geradores para $\overrightarrow{\mathcal{Q}}_{\mathbb{R}^{2} \times \mathbb{C}^{4}}(\tilde{\Gamma})$ sobre $\mathcal{P}_{\mathbb{R}^{2} \times \mathbb{C}^{4}}(\tilde{\Gamma})$.

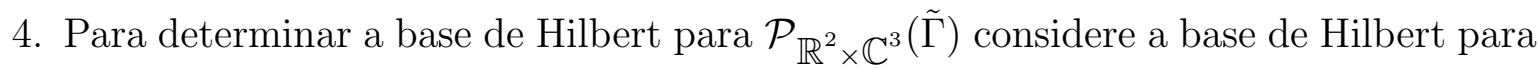
$\mathcal{P}_{\mathbb{R}^{2} \times \mathbb{C}^{4}}\left(\tilde{\Gamma}_{+}\right)$em 5.25 e os operadores $R, S$. Temos

$$
\begin{aligned}
& S\left(v_{1}\right)=\tilde{v}_{1}, \quad S\left(v_{4}\right)=\tilde{v}_{4}, \quad S\left(v_{7}\right)=\tilde{v}_{7}, \quad S\left(v_{8}\right)=\tilde{v}_{8}, \\
& S\left(v_{2}\right)=S\left(v_{3}\right)=S\left(v_{5}\right)=S\left(v_{6}\right)=0, \\
& R\left(v_{2}\right)=v_{2}, \quad R\left(v_{3}\right)=v_{3}, \quad R\left(v_{5}\right)=v_{5}, \quad R\left(v_{6}\right)=v_{6}, \\
& R\left(v_{1}\right)=\left(\frac{1+a_{0}}{2}\right) v_{1}, \\
& R\left(v_{4}\right)=\left(\frac{1+a_{1}^{n_{2}} a_{2}^{n_{1}}}{2}\right) v_{4}, \\
& R\left(v_{7}\right)=\left(\frac{1+a_{3}^{m_{2}} a_{4}^{m_{1}}}{2}\right) v_{7}, \\
& R\left(v_{8}\right)=\left(\frac{1+a_{1}^{n_{2}} a_{2}^{n_{1}} a_{3}^{m_{2}} a_{4}^{m_{1}}}{2}\right) v_{8} .
\end{aligned}
$$

Do Teorema 1.4.2 segue que os elementos abaixo formam uma base de Hilbert para $\mathcal{P} \mathbb{R}^{2} \times \mathbb{C}^{2}(\tilde{\Gamma})$ :

$$
\begin{aligned}
& \left(\frac{1+a_{0}}{2}\right) v_{1}, \quad v_{2}, v_{3}, \quad\left(\frac{1+a_{1}^{n_{2}} a_{2}^{n_{1}}}{2}\right) v_{4}, \quad v_{5}, v_{6}, \quad\left(\frac{1+a_{3}^{m_{2}} a_{4}^{m_{1}}}{2}\right) v_{7} \\
& \left(\frac{1+a_{1}^{n_{2}} a_{2}^{n_{1}} a_{3}^{m_{2}} a_{4}^{m_{1}}}{2}\right) v_{8}, \quad\left(\frac{1-a_{0}}{2}\right) v_{1}^{2}, \quad\left(\frac{1-a_{1}^{n_{2}} a_{2}^{n_{1}}}{2}\right) v_{4}^{2}, \quad\left(\frac{1-a_{3}^{m_{2}} a_{4}^{m_{1}}}{2}\right) v_{7}^{2} \\
& \left(\frac{1-a_{1}^{n_{2}} a_{2}^{n_{1}} a_{3}^{m_{2}} a_{4}^{m_{1}}}{2}\right) v_{8}^{2} \\
& \left(\frac{1-a_{0}}{2}\right)\left(\frac{1-a_{1}^{n_{2}} a_{2}^{n_{1}}}{2}\right) v_{1}(x, z) v_{4}(x, z) \\
& \left(\frac{1-a_{0}}{2}\right)\left(\frac{1-a_{3}^{m_{2}} a_{4}^{m_{1}}}{2}\right) v_{1}(x, z) v_{7}(x, z) \\
& \left(\frac{1-a_{0}}{2}\right)\left(\frac{1-a_{1}^{n_{2}} a_{2}^{n_{1}} a_{3}^{m_{2}} a_{4}^{m_{1}}}{2}\right) v_{1}(x, z) v_{8}(x, z) \\
& \left(\frac{1-a_{1}^{n_{2}} a_{2}^{n_{1}}}{2}\right)\left(\frac{1-a_{3}^{m_{2}} a_{4}^{m_{1}}}{2}\right) v_{4}(x, z) v_{7}(x, z), \\
& \left(\frac{1-a_{1}^{n_{2}} a_{2}^{n_{1}}}{2}\right)\left(\frac{1-a_{1}^{n_{2}} a_{2}^{n_{1}} a_{3}^{m_{2}} a_{4}^{m_{1}}}{2}\right) v_{4}(x, z) v_{8}(x, z)
\end{aligned}
$$




$$
\left(\frac{1-a_{3}^{m_{2}} a_{4}^{m_{1}}}{2}\right)\left(\frac{1-a_{1}^{n_{2}} a_{2}^{n_{1}} a_{3}^{m_{2}} a_{4}^{m_{1}}}{2}\right) v_{7}(x, z) v_{8}(x, z) .
$$

Com os geradores em mãos, selecionamos a antissimetria $\psi$ que queremos usar, além dos valores de ressonância, e determinamos a forma geral $\mathbf{Z}_{2}^{\phi} \times \mathbf{Z}_{2}^{\psi}$-reversível-equivariante. Note novamente que quando $\phi=\psi$ recaímos no caso $\mathbf{Z}_{2}^{\phi}$-reversível-equivariante.

É possível considerar o sistema (5.1) com $L=(d h)_{0}$ tendo somente autovalores imaginários, ou seja, $L$ sem a parte nilpotente. Neste caso, todas as formas normais calculadas neste capítulo podem ser reescritas a $\mathbb{C}^{n}$, para algum $n$. Para isto, basta desconsiderar as variáveis $x_{1}$ e $x_{2}$, juntamente com todos os geradores que possuem estas variáveis. Por exemplo, no Teorema (5.3.3), a forma normal do Tipo $D$ fica:

$$
\begin{aligned}
\dot{z}_{1}= & -i \omega_{1} z_{1}+i z_{1} f_{0}(X)+\bar{z}_{1}^{n_{2}-1} z_{2}^{n_{2}} \operatorname{Im}\left(z_{1}^{n_{2}} \bar{z}_{2}^{n_{1}}\right) f_{5}(X)+i \bar{z}_{1}^{n_{2}-1} z_{2}^{n_{2}} \operatorname{Re}\left(z_{1}^{n_{2}} \bar{z}_{2}^{n_{1}}\right) f_{1}(X)+ \\
& +z_{1} \operatorname{Re}\left(z_{1}^{n_{2}} \bar{z}_{2}^{n_{1}}\right) \operatorname{Im}\left(z_{1}^{n_{2}} \bar{z}_{2}^{n_{1}}\right) f_{2}(X) \\
\dot{z}_{2}= & -i \omega_{2} z_{2}+i z_{2} f_{3}(X)+z_{1}^{n_{2}} \bar{z}_{2}^{n_{1}-1} \operatorname{Im}\left(z_{1}^{n_{2}} \bar{z}_{2}^{n_{1}}\right) f_{4}(X)+i z_{1}^{n_{2}} \bar{z}_{2}^{n_{1}-1} \operatorname{Re}\left(z_{1}^{n_{2}} \bar{z}_{2}^{n_{1}}\right) f_{5}(X)+ \\
& +z_{2} \operatorname{Re}\left(z_{1}^{n_{2}} \bar{z}_{2}^{n_{1}}\right) \operatorname{Im}\left(z_{1}^{n_{2}} \bar{z}_{2}^{n_{1}}\right) f_{6}(X)
\end{aligned}
$$

para $f_{i}: \mathbb{R}^{3}, 0 \rightarrow \mathbb{R}, \quad 0 \leq i \leq 6, \quad X=\left(\left|z_{1}\right|^{2},\left|z_{2}\right|^{2}, \operatorname{Re}^{2}\left(z_{1}^{n_{2}} \bar{z}_{2}^{n_{1}}\right)\right)$.

Observamos que os pares de involuções $(\phi, \psi)$ que anti-comutam com $L$ dada em $(5.2)$ foram calculados com a hipótese de que $\omega_{i}^{2} \neq \omega_{j}^{2}$ para $i \neq j$ em $\{1, \ldots, n\}$. Porém, tais pares ainda anti-comutam com $L$ caso $\omega_{i}=-\omega_{j}$ para algum $i \neq j$ em $\{1, \ldots, n\}$. Esta condição corresponde ao caso 1 : 1-ressonante que também é de interesse em sistemas dinâmicos. Para este caso, as formas normais obtidas neste capítulo ainda são válidas.

Observação 5.3.6. Finalizamos este capítulo observando que se $\langle\phi, \psi\rangle$ é um grupo finito não abeliano, este é um grupo diedral $\boldsymbol{D}_{m}$ para algum $m \geq 3$. Este é um caso que nos despertou interesse recentemente, o qual pretendemos lidar no futuro breve. Mais especificamente, este caso leva à teoria invariante para o grupo $\left(\boldsymbol{S} \times \boldsymbol{Z}_{m}\right) \rtimes \boldsymbol{Z}_{2}$ uma vez que, pelo Teorema 3.3.1 é preciso determinar geradores para $\overrightarrow{\mathcal{Q}}_{V}\left(\left(\boldsymbol{S} \times \boldsymbol{Z}_{m}\right) \rtimes \boldsymbol{Z}_{2}\right)$ sobre o anel $\mathcal{P}_{V}\left(\left(\boldsymbol{S} \times \boldsymbol{Z}_{m}\right) \rtimes \boldsymbol{Z}_{2}\right)$ para o cálculo da forma normal $\boldsymbol{D}_{m}$-reversível-equivariante. Acreditamos que este problema possa ser tratado de forma similar ao caso $\boldsymbol{Z}_{2} \times \boldsymbol{Z}_{2}$-reversivelequivariante, partindo inicialmente dos geradores de $\overrightarrow{\mathcal{P}}_{V}(\boldsymbol{S})$ e $\mathcal{P}_{V}(\boldsymbol{S})$ apenas, e aplicando resultados recentemente obtidos em [4]. 


\section{Referências Bibliográficas}

[1] Antoneli, F., Dias, A. P. S. e Matthews, P. C. [2007] Invariants, Equivariants and Characters in Symmetric Bifurcation Theory. Proceedings of the Royal Society of Edinburgh. Section A, Mathematics 137, 01-36.

[2] Antoneli, F., Baptistelli, P. H., Dias, A. P. S. e Manoel, M. G. [2009] Invariant theory and reversible equivariant vector fields. Journal of Pure and Applied Algebra 213, 649-663.

[3] Baptistelli, P. H. e Manoel, M. G. [2008] The classification of reversible-equivariant steady-state bifurcations on self-dual spaces. Mathematical Proceedings of the Cambridge Philosophical Society (Print) 145, 379-401.

[4] Baptistelli, P. H. e Manoel, M. G. [2013] Invariants and relative invariants under compact Lie groups. A aparecer em Journal of Pure and Applied Algebra (Print).

[5] Baptistelli, P. H. e Tempesta, P. [2012] Teoria invariante em sistemas dinâmicos reversíveis equivariantes. Dissertação de mestrado, Universidade Estadual de Maringá.

[6] Belitskii, G. R. [2002] $C^{\infty}$-normal forms of local vector fields. Symmetry and perturbation theory. Acta Applicandae Mathematicae 70, 23-41.

[7] Birkhoff, G. D. [1927] Dynamical Systems. American Mathematical Society, Colloquium Publication IX, New York. 
[8] Bröcker, T. e Dieck, T. tom [1995] Representations of compact Lie groups. Graduate Texts in Mathematics 98, Spreing-Verlag, Berlin-Heidelberg, New York.

[9] Broer, H. W. [1981] Formal normal form theorems for vector fields and some consequences for bifurcation in the volume preserving case. In "Dynamical systems and turbulence"(Rand, D. A. and Young L. S.), Lecture Notes in Mathematics 898, Springer, Berlim.

[10] Bruce, J. W. , du Plessis, A. A. e Wall, C. T. C. [1987] Determinacy and unipotency. Inventiones mathematicae $\mathbf{8 8}, 521-554$.

[11] Bruce, J. W, Kirk, N. P e du Plessis, A. A. [1997] Complete transversals and the classification of singularities. Nonlinearity 10, 253-275.

[12] Buono, P. L., Lamb, J. S. W. e Roberts, R. M. [2007] Bifurcation and branching of equilibria of reversible equivariant vector fields. Nonlinearity 21, 625-660.

[13] Buzzi, C. A. e Lamb, J. S. W. [2005] Reversible equivariant Hopf bifurcation. Archive for Rational Mechanics and Analysis 175, 39-84.

[14] Buzzi, C. A., Llibre, J. e Medrado, J. C. [2007] Periodic orbits for a class of reversible quadratic vector field on $\mathbb{R}^{3}$. Journal of Mathematical Analysis and Applications $\mathbf{3 3 5}$, 1335-1346.

[15] Buzzi, C. A., Llibre, J., Medrado, J. C. e Torregrosa, J. [2009] Bifurcation of limit cycles from a centre in $\mathbb{R}^{4}$ in resonance $1: N$. Dynamical Systems $\mathbf{2 4}$, no. 1, 123-137.

[16] Barreira, L., Llibre, J. e Valls, C. [2012] Limit cycles from a four-dimensional centre in $\mathbb{R}^{m}$ in resonance $p: q$. Dynamical Systems $\mathbf{2 7}(4), 459-474$.

[17] Buzzi, C. A., Roberto, L. A. e Teixeira, M. A. [2010] Branching of periodic orbits in reversible Hamiltonian systems. Real and complex singularities, London Mathematical Society Lecture Note Series 380, Cambridge Univ. Press, Cambridge, 46-70.

[18] Carmona, V. , Fernández-Sánchez, F. e Teruel, A. E. [2008] Existence of a reversible $T$-Point heteroclinic cycle in a piecewise linear version of the Michelson system. Journal on Applied Dynamical Systems 7(3), 1032-1048. 
[19] Castro, S. B. S. D. e du Plessis, A. A [2005] Intrinsic comlete transversal and the recognition of equivariant bifurcations. EQUADIFF 2003, World Sci. Publ., Hackensack, NJ, 458-463.

[20] Champneys, A.R. [1998] Homoclinic orbits in reversible systems and their applications in mechanics, fluids and optics. Physica D: Nonlinear Phenomena 112, Issues 1-2, 158-186.

[21] Chow, S. N., LI, C. e Wang, D [1994] Normal forms and bifurcation of planar vector fields. Cambridge University-Press.

[22] Cima, A., Llibre, J. e Teixeira, M. A. [2008] Hopf bifurcation in presence of $1: 3$ resonance. Advanced Nonlinear Studies 8(3), 619-632.

[23] Damon, J. [1984] The unfolding and determinacy theorems for subgroups of $\mathcal{A}$ and $\mathcal{K}$. Memoirs of the American Mathematical society 306.

[24] Dias, A. P. S. [1997] Bifurcations with wreath product simmetry. Phd thesis, University of Warwick, Inglaterra.

[25] Dionne, B., Golubitsky, M. e Stewart, I. [1996] Coupled cells with internal symmetry Part I: wreath products. Nonlinearity $\mathbf{9}, 559-574$.

[26] Dionne, B., Golubitsky, M e Stewart, I. [1996] Coupled cells with internal symmetry. II. Direct products. Nonlinearity 9 (2), 575-599.

[27] Devaney, R. L. [1976] Reversible diffeomorphisms and flows. Transactions of the American Mathematical Society, 218, 89-113.

[28] Dulac, H. [1912] Solution d'un systeme d'equations differentielles dans le voisinage de valeurs singulieres. Bulletin de la Société Mathématique de France 40, 324-83.

[29] Elphick, C., Tirapegui, E., Brachet, M. E., Coullet, P., e Iooss, G. [1987] A simple global characterization for normal forms of singular vector fields. Physica D: Nonlinear Phenomena 29, 95-127.

[30] Gaffney, T. e du Plessis, A. [1982] More on the determinacy of smooth map-germs. Inventiones Mathematicae 66(1), 137-163. 
[31] Gaffney, T. [1983] The structure of $T \mathcal{A}(f)$, classification and an application to differential geometry. Singularities, Part 1 (Arcata, Calif., 1981), 409-427, Proceedings of Symposia in Pure Mathematics 40, American Mathematical Society, Providence, RI.

[32] GAP [2013] Groups, Algorithms and Programming - a System for Computational Discrete Algebra (http://www.gap-system.org/).

[33] Gibson, C. G. [1979] Singular points of smooth mappings. Research Notes in Mathematics Series.

[34] Golubitsky, M. e Schaeffer, D. [1985] Singularities and Groups in Bifurcation Theory. Vol. I, Appl.Math. Sci. 51, Springer-Verlag, New York.

[35] Golubitsky, M., Stewart, I. e Schaeffer, D. [1988] Singularities and Groups in Bifurcation Theory. Vol. II, Appl. Math. Sci. 69, Springer-Verlag, New York.

[36] Hochschild, G. [1965] The structure of Lie groups. Holden-Day, San Francisco.

[37] Iooss, G. e Adelmeyer, M. [1999] Topics in bifurcation theory and applications, Advanced Series in Nonlinear Dynamics, Vol. 3, 2ed, Singapore.

[38] Jacquemard, A. e Teixeira, M. A. [2000] On singularities of reversible mappings in $\mathbb{R}^{3}$. Dynamics of Continuous, Discrete and Impulsive Systems7, 65-96.

[39] Jacquemard, A. e Teixeira, M. A. [2002] Effective algebraic geometry and normal forms of reversible mappings. Revista Matematica Complutense 15(1), 31-55.

[40] Kirk, N. P. [2000] Computational Aspects of Classifying Singularities. LMS Journal of Computation and Mathematics, 207-228.

[41] Lamb, J. S. W. [1992] Reversing symmetries in dynamical systems. Journal of Physics A: Mathematical and General 25, 925-937.

[42] Lamb, J. S. W. e Roberts, R. M. [1999] Reversible equivariant linear systems. Journal of Differential Equations 159, 239-279.

[43] Lamb, J. S. W., Teixeira, M. A. e Webster, K. N. [2005] Heteroclinic bifurcations near Hopf-zero bifurcation in reversible vector fields in $R^{3}$. Journal of Differential Equations 219(1), 78-115. 
[44] Lima, M. F. S. e Teixeira, M. A. [2009] Families of periodic orbits in resonant reversible systems. Bulletin of the Brazilian Mathematical Society, New Series 40(4), 511-537.

[45] Lima, M. F. S. e Teixeira, M. A. [2013] Homoclinic orbits in degerative reversibleequivariant systems in $\mathbb{R}^{6}$. A aparecer em Journal of Mathematical Analysis and Applications, Elsevier.

[46] Mancini, S., Manoel, M. G. e Teixeira, M. A. [2005] Divergent diagrams of folds and simultaneous conjugacy of involutions. Discrete and Continuous Dynamical Systems, Estados Unidos, 12 (4), 657-674.

[47] Martins, R. M. e Teixeira, M. A. [2011] Reversible equivariant systems and matricial equations, Anais da Academia Brasileira de Ciências 83 (2), 375-390.

[48] Melbourne, I. [1986] A singularity theory analysis of bifurcation problems with octahedral symmetry. Dynamics and Stability of System 1 (4), 293-321.

[49] Melbourne, I. [1988] The recognition problem for equivariant singularities. Nonlinearity 1 (1), 215-240.

[50] Mereu, A. C. e Teixeira, M. A. [2013] Reversibility and branching of periodic orbits. Discrete and Continuous Dynamical Systems, 33, 1177-1199.

[51] Montgomery, D. e Zippin, L. [1955] Topological Transformation Groups, Interscience, New York.

[52] Nachbin, L. [1976] The Haar Integral, (Huntington, New York).

[53] Poincaré, H. [1879] Thesis; also Oeuvres I, 59-129, Gauthier Villars, Paris, 1928.

[54] Sattinger, D. H. [1979] Group Theoretic Methods in bifurcation Theory. Lecture Notes in Mathematics 762, Springer, Berlin, New York.

[55] Singular - versão 3-0-4 [2008](http://www.singular.uni-kl.de/)

[56] Sturmfels, B. [1993] Algorithms in Invariant Theory. Texts and Monographs in Symbolic Computation, Springer-Verlag, Austria. 
[57] Takens, F. [1973] Normal forms for certain singularities of vector fields. An. Inst. Fourier 23(2), 163-195.

[58] Tari, F. [1999] Singularidades de Aplicações diferenciáveis, Notas Didáticas do ICMCUSP, no. 34.

[59] Wall, C. T. C. [1981] Finite Determinacy of Smooth Map-Germs. Bulletin London Mathematical Society 13, 481-539.

[60] Wall, C. T. C. [1995] Classification and stability of singularities of smooth maps. Singularity theory (Trieste, 1991), 920-952, World Sci. Publ., River Edge, NJ.

[61] Worfolk, P. A. [1994] Zeros of equivariant vector fields: Algorithms for an invariant approach. Journal of Symbolic Computation, 17, 487-511. 


\section{Índice Remissivo}

$k$-jatos, 44

versal, 46

Órbita, 45

Equivalência, 45, 51

Ação, 8

Espaço

de $\tilde{\mathcal{G}}, 50$

$J^{k}, 44$

de $\mathcal{G}, 47$

$\Gamma$-isomorfo, 13

de $\tilde{\mathcal{K}}, 45$

$\overrightarrow{\mathcal{F}}_{V k+1}(\Gamma), 51$

de $\mathbf{T}, 26$

$J^{k}\left(\Gamma_{\sigma}\right), 51$

de $\mathbf{T}^{n}, 26$

tangente, 45, 48, 52

diagonal, 24

do produto semi-direto, 22

dual, 12

ortogonal, 10

Antissimetria, 12

$\delta, 12,14$

Aplicação

$\Gamma$-equivariante, 10

$\Gamma$-reversível-equivariante, 12

Fórmulas de Molien, 26, 27

Forma normal

de Belitskii, 33

reversível equivariante, 36

Formalmente conjugado, 32,33

Função

$$
\begin{aligned}
& \Gamma \text {-anti-invariante, } 12 \\
& \Gamma \text {-invariante, } 10
\end{aligned}
$$

Células acopladas, 29

Grupo

Caracter, 18, 26

$$
\begin{aligned}
& J^{k+1} \mathcal{G}, 47 \\
& J^{k+1} \tilde{\mathcal{G}}, 51 \\
& \tilde{\mathcal{K}}, 45 \\
& \mathbf{S}=\mathbf{R} \times \mathbf{T}^{k}, 59
\end{aligned}
$$


$\mathbf{S} \rtimes \Gamma, 36$

S, 34

Hilbert

base de, 10

série de, 16, 17

Ideal maximal de $\mathcal{E}_{V}, 44$

Integral de Haar, 9, 37

normalizada, 9

Involução, 55

Jordan-Chevalley, 59

Método

de Elphick, 33, 34

Molien

fórmulas de, 16, 18

Mudanças de coordenadas, 32

Não ressonante, 59

Operador

homológico, 33, 34, 48, 50

linear, 48

Produto

direto, 23

semi-direto, 22

Projeção $\pi, 37$

Representação, 8

$\Gamma$-isomorfas, 18

$\sigma$-dual, 12

auto-dual, 13

diagonal, 24

do produto semi-direto, 23
Ressonante, 62

Reynolds

operadores de, 13, 14, 35

Simetria, 12

Teorema

da transversal completa, 47

reversível equivariante, 51

de Fubibi, 13

de Hilbert-Weyl, 10

de Molien, 18

de Molien equivariante, 18

de Poènaru, 11

de Schwarz, 11

do desdobramento, 46 